\title{
Cochrane
}

Library

Cochrane Database of Systematic Reviews

\section{Urinary biomarkers for the non-invasive diagnosis of endometriosis} (Review)

Liu E, Nisenblat V, Farquhar C, Fraser I, Bossuyt PMM, Johnson N, Hull ML

Liu E, Nisenblat V, Farquhar C, Fraser I, Bossuyt PMM, Johnson N, Hull ML. Urinary biomarkers for the non-invasive diagnosis of endometriosis.

Cochrane Database of Systematic Reviews 2015, Issue 12. Art. No.: CD012019. DOI: 10.1002/14651858.CD012019.

www.cochranelibrary.com 
TABLE OF CONTENTS

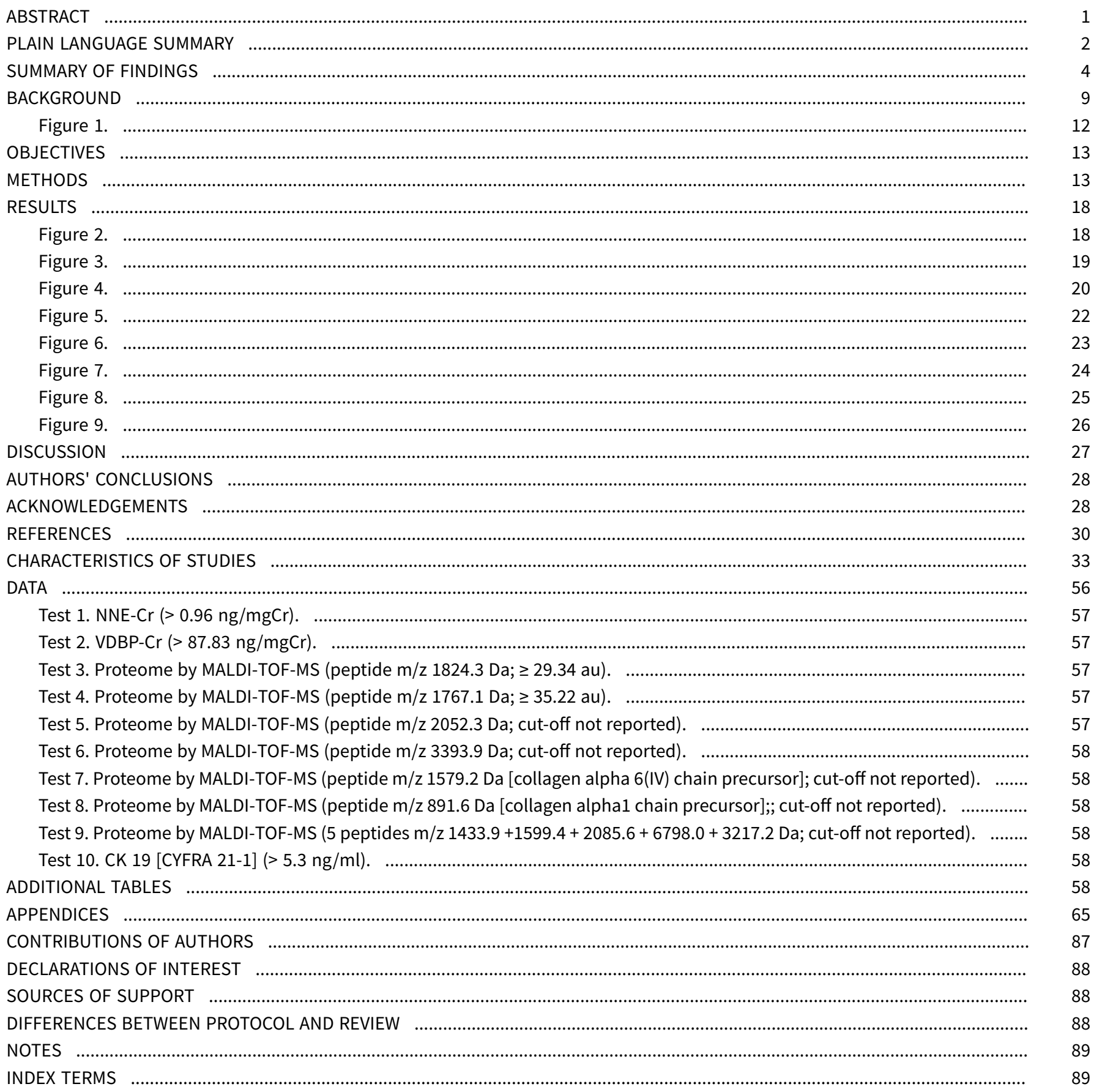


[Diagnostic Test Accuracy Review]

\section{Urinary biomarkers for the non-invasive diagnosis of endometriosis}

Emily Liu1 ${ }^{1 a}$, Vicki Nisenblat ${ }^{2 b}$, Cindy Farquhar ${ }^{3}$, Ian Fraser ${ }^{4}$, Patrick MM Bossuyt ${ }^{5}$, Neil Johnson², M Louise Hull2

1 Fertility PLUS, Auckland District Health Board, Auckland, New Zealand. 2Discipline of Obstetrics and Gynaecology, School of Medicine, Robinson Research Institute, The University of Adelaide, Adelaide, Australia. ${ }^{3}$ Department of Obstetrics and Gynaecology, University of Auckland, Auckland, New Zealand. 'School of Women's and Children's Health, Royal Hospital for Women, University of New South Wales, Sydney, Australia. ${ }^{5}$ Department of Clinical Epidemiology, Biostatistics and Bioinformatics, Academic Medical Center, University of Amsterdam, Amsterdam, Netherlands

a Joint first author. b Joint first author

Contact: Vicki Nisenblat, Discipline of Obstetrics and Gynaecology, School of Medicine, Robinson Research Institute, The University of Adelaide, Level 6, Medical School North,, Frome Rd, Adelaide, SA, 5005, Australia.vnisenblat@gmail.com.

Editorial group: Cochrane Gynaecology and Fertility Group.

Publication status and date: New, published in Issue 12, 2015.

Citation: Liu E, Nisenblat V, Farquhar C, Fraser I, Bossuyt PMM, Johnson N, Hull ML. Urinary biomarkers for the non-invasive diagnosis of endometriosis. Cochrane Database of Systematic Reviews 2015, Issue 12. Art. No.: CD012019. DOI: 10.1002/14651858.CD012019.

Copyright () 2015 The Cochrane Collaboration. Published by John Wiley \& Sons, Ltd.

\section{A B S T R A C T}

\section{Background}

About $10 \%$ of reproductive-aged women suffer from endometriosis which is a costly chronic disease that causes pelvic pain and subfertility. Laparoscopy is the 'gold standard' diagnostic test for endometriosis, but it is expensive and carries surgical risks. Currently, there are no simple non-invasive or minimally-invasive tests available in clinical practice that accurately diagnoses endometriosis.

\section{Objectives}

1. To provide summary estimates of the diagnostic accuracy of urinary biomarkers for the diagnosis of pelvic endometriosis compared to surgical diagnosis as a reference standard.

2. To assess the diagnostic utility of biomarkers that could differentiate ovarian endometrioma from other ovarian masses.

Urinary biomarkers were evaluated as replacement tests for surgical diagnosis and as triage tests to inform decisions to undertake surgery for endometriosis.

\section{Search methods}

The searches were not restricted to particular study design, language or publication dates. We searched the following databases to $20 \mathrm{April}$ - 31 July 2015: CENTRAL, MEDLINE, EMBASE, CINAHL, PsycINFO, Web of Science, LILACS, OAIster, TRIP and ClinicalTrials.gov (trial register). MEDION, DARE, and PubMed were also searched to identify reviews and guidelines as reference sources of potentially relevant studies. Recently published papers not yet indexed in the major databases were also sought. The search strategy incorporated words in the title, abstract, text words across the record and the medical subject headings (MeSH) and was modified for each database.

\section{Selection criteria}

Published peer-reviewed, randomised controlled or cross-sectional studies of any size were considered, which included prospectively collected samples from any population of reproductive-aged women suspected of having one or more of the following target conditions: ovarian, peritoneal or deep infiltrating endometriosis (DIE). We included studies comparing the diagnostic test accuracy of one or more urinary biomarkers with surgical visualisation of endometriotic lesions. 


\section{Data collection and analysis}

Two authors independently collected and performed a quality assessment of the data from each study. For each diagnostic test, the data were classified as positive or negative for the surgical detection of endometriosis and sensitivity and specificity estimates were calculated. If two or more tests were evaluated in the same cohort, each was considered as a separate data set. The bivariate model was used to obtain pooled estimates of sensitivity and specificity whenever sufficient data sets were available. The predetermined criteria for a clinically useful urine test to replace diagnostic surgery was one with a sensitivity of $94 \%$ and a specificity of $79 \%$ to detect endometriosis. The criteria for triage tests were set at sensitivity of equal or greater than $95 \%$ and specificity of equal or greater than $50 \%$, which in case of negative result rules out the diagnosis (SnOUT test) or sensitivity of equal or greater than $50 \%$ with specificity of equal or greater than $95 \%$, which in case of positive result rules the diagnosis in (SpIN test).

\section{Main results}

We included eight studies involving 646 participants, most of which were of poor methodological quality. The urinary biomarkers were evaluated either in a specific phase of menstrual cycle or irrespective of the cycle phase. Five studies evaluated the diagnostic performance of four urinary biomarkers for endometriosis, including three biomarkers distinguishing women with and without endometriosis (enolase 1 (NNE); vitamin D binding protein (VDBP); and urinary peptide profiling); and one biomarker (cytokeratin 19 (CK 19)) showing no significant difference between the two groups. All of these biomarkers were assessed in small individual studies and could not be statistically evaluated in a meaningful way. None of the biomarkers met the criteria for a replacement test or a triage test. Three studies evaluated three biomarkers that did not differentiate women with endometriosis from disease-free controls.

\section{Authors' conclusions}

There was insufficient evidence to recommend any urinary biomarker for use as a replacement or triage test in clinical practice for the diagnosis of endometriosis. Several urinary biomarkers may have diagnostic potential, but require further evaluation before being introduced into routine clinical practice. Laparoscopy remains the gold standard for the diagnosis of endometriosis, and diagnosis of endometriosis using urinary biomarkers should only be undertaken in a research setting.

\section{PLAIN LANGUAGE SUMMARY}

\section{Urinary biomarkers for the non-invasive diagnosis of endometriosis}

\section{Review Question}

How accurate are urine test in detecting endometriosis? Can any urine test be accurate enough to replace or reduce the need for surgery in the diagnosis of endometriosis?

\section{Background}

Women with endometriosis have endometrial tissue (the tissue that lines the womb and is shed during menstruation) growing outside the womb within the pelvic cavity. This tissue responds to reproductive hormones causing painful periods, chronic lower abdominal pain and difficulty conceiving. Currently, the only reliable way of diagnosing endometriosis is to perform laparoscopic surgery and visualise the endometrial deposits inside the abdomen. Because surgery is risky and expensive, urine tests have been evaluated for their ability to detect endometriosis non-invasively. An accurate urine test could lead to the diagnosis of endometriosis without the need for surgery; or it could reduce the need for diagnostic surgery, so only women who were most likely to have endometriosis would require it. Other noninvasive ways of diagnosing endometriosis using blood, imaging, endometrial and combination tests are evaluated in separate Cochrane reviews from this series.

\section{Study characteristics}

The evidence included in this review is current to July 2015. We included eight studies involving 646 participants. All studies evaluated reproductive-aged women who were undertaking diagnostic surgery to investigate symptoms of endometriosis or for other indications. Five studies evaluated the diagnostic accuracy of four urinary biomarkers, including four biomarkers that were expressed differently in women with and without endometriosis and one showing no difference between the two groups. Three other studies just identified three biomarkers that did not distinguish the two groups.

\section{Key results}

None of the assessed biomarkers, including cytokeratin 19 (CK 19), enolase 1 (NNE), vitamin D binding protein (VDBP) and urinary peptide profiling have been evaluated by enough studies to provide a meaningful assessment of test accuracy. None of the tests were accurate enough to replace diagnostic surgery. Several studies identified biomarkers that might be of value in diagnosing endometriosis, but there are too few reports to be sure of their diagnostic benefit. There is not enough evidence to recommend any urinary biomarker for use in clinical practice for the diagnosis of endometriosis.

\section{Quality of the evidence}


Generally, the reports were of low methodological quality and urine tests were only assessed in small individual studies.

\section{Future research}

More high quality research trials are needed to accurately assess the diagnostic potential of urinary biomarkers identified in small numbers of studies as having value in detecting endometriosis. 
SUMMARY OF FINDINGS

Summary of findings 1 . Biomarkers evaluated as a diagnostic test for endometriosis

Review What is the diagnostic accuracy of the urinary biomarkers in detecting pelvic endometriosis [peritoneal endometriosis, endometrioma, DIE]? question

Importance A simple and reliable non-invasive test for endometriosis, with the potential to either replace syrgery or to triage patients in order to reduce surgery, would minimise surgical risk and reduce diagnostic delay

Patients Reproductive-aged women 1) with suspected endometriosis or 2) with persistent ovarian mass or 3) undergoing infertility workup or gynaecological laparoscopy

Settings Hospitals (public or private of any level): outpatient clinics (general gynaecology, reproductive medicine, pelvic pain); research laboratories

Reference Visualisation of endometriosis at surgery (laparoscopy or laparotomy) with or without histological confirmation

Study de- $\quad$ Cross sectional studies with a 'single-gate' design $(n=4)$ or a 'two-gate' design $(n=1)$; prospective enrolment; a single study could assess more than one test

sign

Risk of bias Overall judgement: Poor quality of most of the studies (no study had a 'low risk' assessment in all 4 domains)

Patient selection bias: High risk - 1 study; Unclear risk - 4 studies; Low risk - 0 studies

Index test interpretation bias: High risk - 5 studies; Unclear risk - 0 studies; Low risk - 0 studies

Reference standard interpretation bias: High risk - 0 studies; Unclear risk - 1 study; Low risk - 4 studies

Flow and timing selection bias: High risk - 1 study; Unclear risk - 0 studies; Low risk - 4 studies

Applicabili- Concerns regarding patient selection: High concern - 3 studies; Unclear concern - 0 studies; Low concern 2 studies

ty concerns

Concerns regarding index test: High concern - 0 studies; Unclear concern - 0 studies; Low concern - 5 studies

Concerns regarding reference standard: High concern - 0 studies; Unclear concern - 0 studies; Low concern - 5 studies

\begin{tabular}{|c|c|c|c|c|c|c|c|}
\hline \multirow[t]{2}{*}{ Biomarker } & \multirow{2}{*}{$\begin{array}{l}\mathrm{N} \text { of stud- } \\
\text { ies; } \\
\mathrm{N} \text { of women }\end{array}$} & \multicolumn{4}{|l|}{ Outcomes } & \multirow{2}{*}{$\begin{array}{l}\text { Diagnostic estimates } \\
{[95 \% \mathrm{Cl}]}\end{array}$} & \multirow[t]{2}{*}{ Implications } \\
\hline & & $\begin{array}{l}\text { True posi- } \\
\text { tives } \\
\text { (en- } \\
\text { dometriosis }\end{array}$ & $\begin{array}{l}\text { False nega- } \\
\text { tives (incor- } \\
\text { rectly }\end{array}$ & $\begin{array}{l}\text { True nega- } \\
\text { tives (dis- } \\
\text { ease-free) }\end{array}$ & $\begin{array}{l}\text { False posi- } \\
\text { tives (incor- } \\
\text { rectly }\end{array}$ & & \\
\hline
\end{tabular}




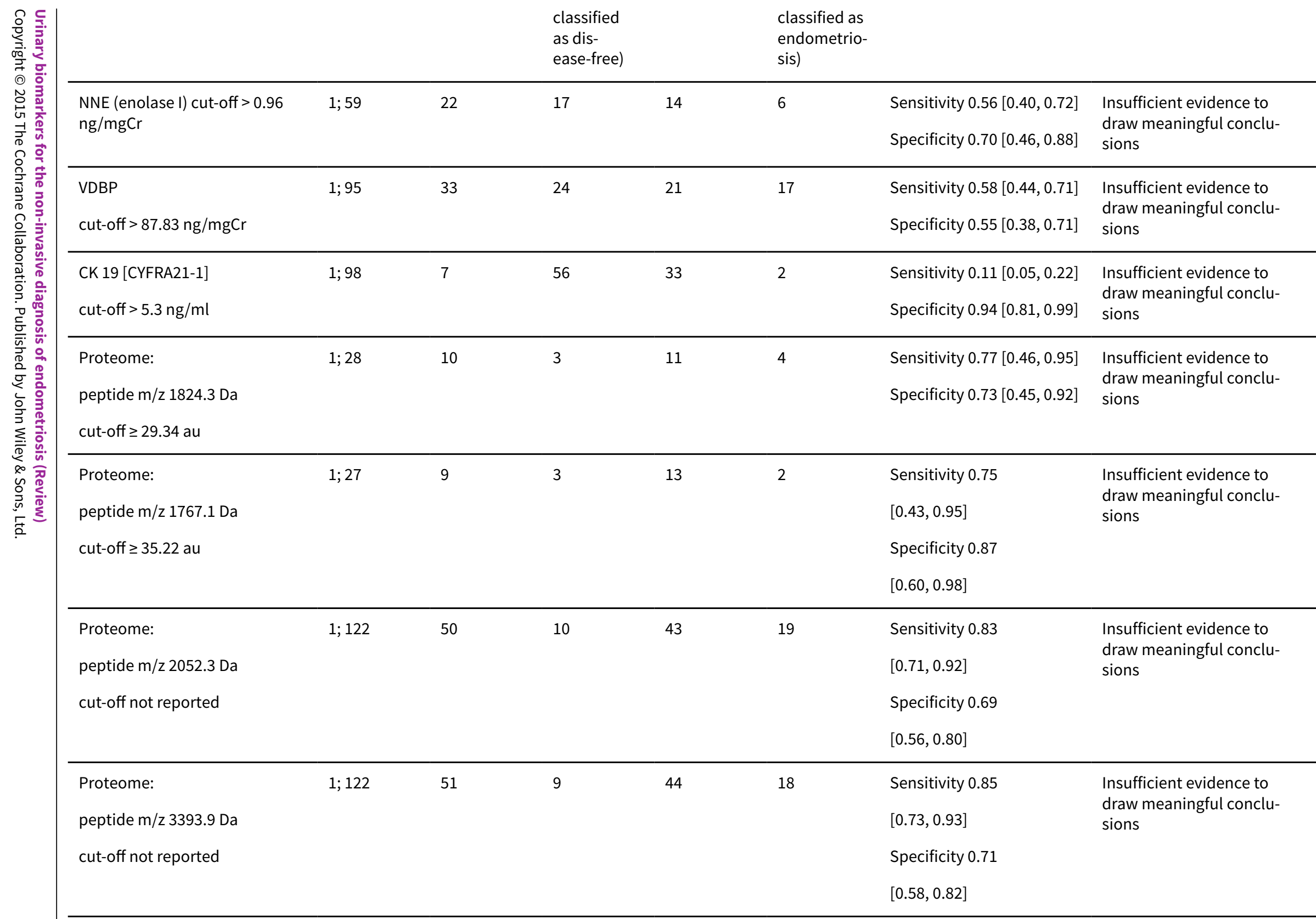




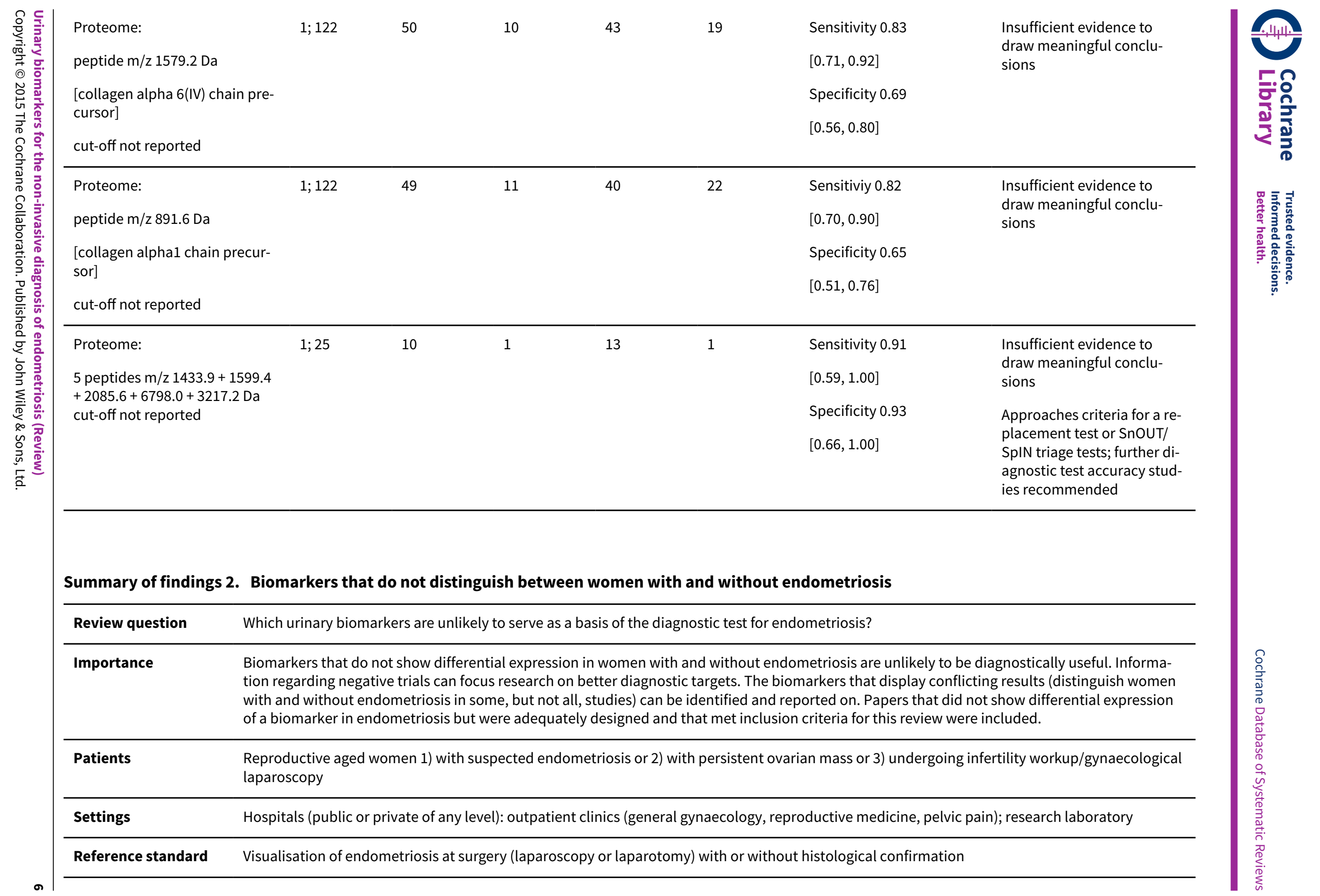




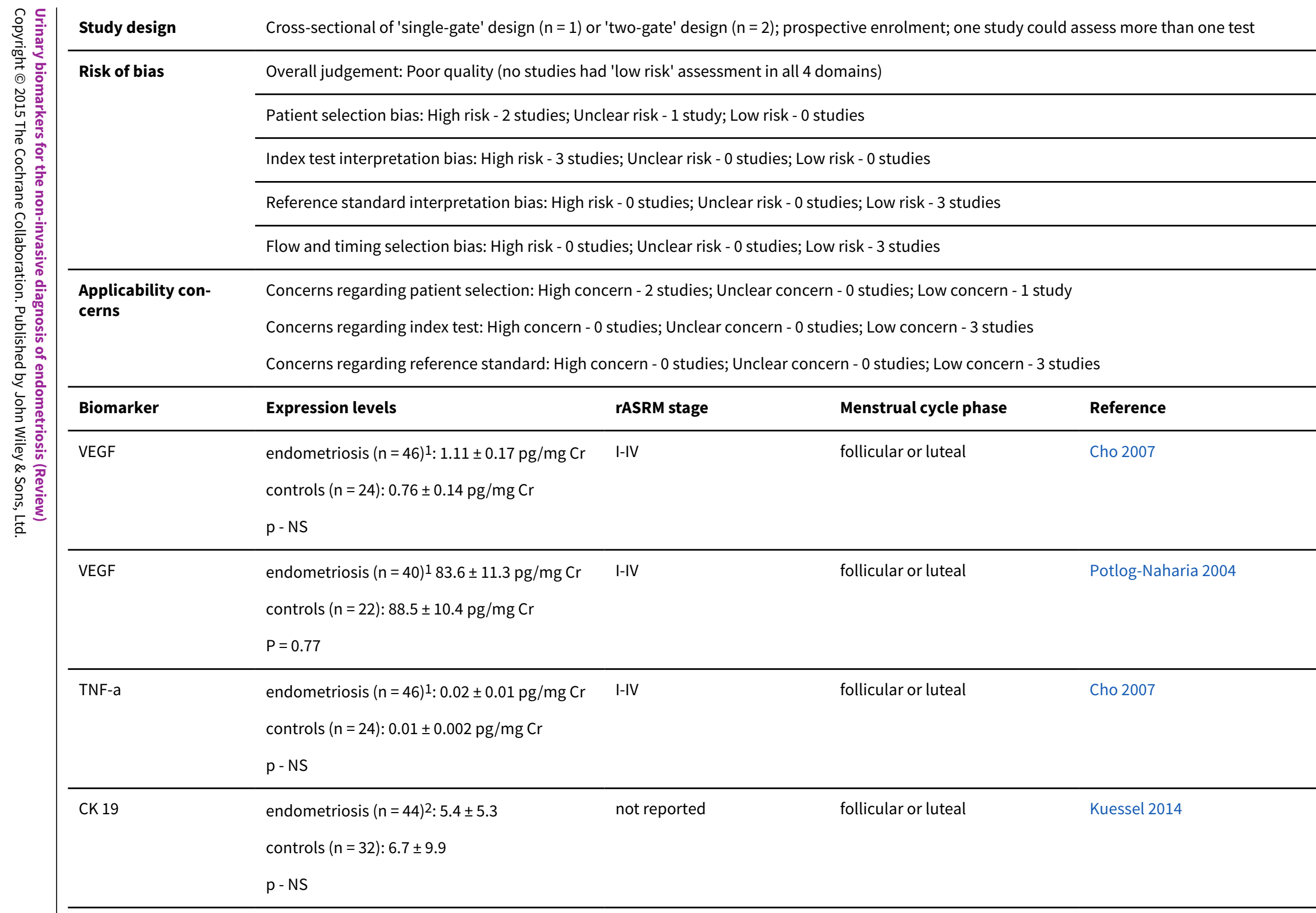

1 mean \pm SEM 


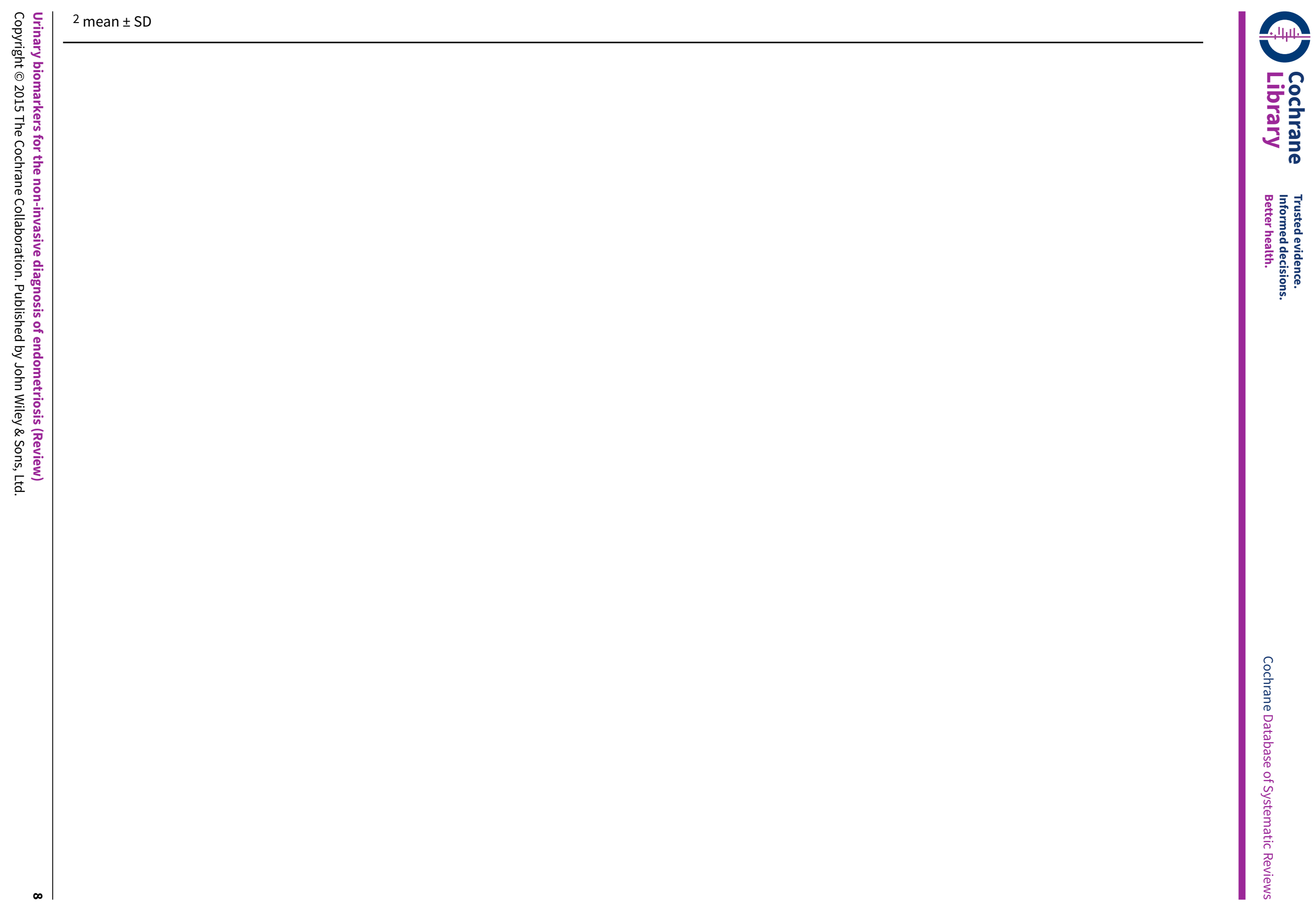




\section{B A C K G R O U N D}

\section{Target condition being diagnosed}

\section{Endometriosis}

Endometriosis is defined as an inflammatory condition characterised by endometrial-like tissue at sites outside the uterus (Johnson and Hummelshoj 2013). Endometriotic lesions can occur at different locations, including the pelvic peritoneum and the ovary; or they can penetrate pelvic structures below the surface of peritoneum (defined as deeply infiltrating endometriosis (DIE)). Each of these types of endometriosis are thought to represent a separate clinical entity, but can coexist in the same woman. Rarely, endometriotic implants can be found at more distant sites, including lung, liver, pancreas and operative scars, with consequent variations in presenting symptoms.

Endometriosis afflicts $10 \%$ of reproductive-aged women causing dysmenorrhoea (painful periods), dyspareunia (painful intercourse), chronic pelvic pain and infertility (Vigano 2004). The clinical presentation can vary from asymptomatic and unexplained infertility to severe dysmenorrhoea and chronic pain. These symptoms can occur with bowel or urinary symptoms, an abnormal pelvic examination or the presence of a pelvic mass, however no symptom is specific to endometriosis. The prevalence of endometriosis in symptomatic population is reported as $35-50 \%$ (Giudice 2004).

Women with endometriosis are also at increased risk of developing several cancers (Somigliana 2006) and autoimmune disorders (Sinaii 2002). The presence of disease is associated with changes in the immune response, vascularisation, neural function, the peritoneal environment and the eutopic endometrium, suggesting that endometriosis is a systemic, rather than localized, condition (Giudice 2004). Endometriosis has a profound effect on psychological and social well being and imposes a substantial economic burden on society. Women with endometriosis incur significant direct medical costs from diagnostic and therapeutic surgeries, hospital admissions and fertility treatments, however these costs are superseded by the indirect costs of endometriosis including absenteeism and loss of productivity (Gao 2006; Simoens 2012). In the USA, the financial burden of endometriosis is estimated at USD 12,419 per woman (Simoens 2012).

Although the pathogenesis of endometriosis has not been fully elucidated, it is commonly thought that endometriosis occurs when endometrial tissue contained within the menstrual fluid flows back through the fallopian tubes and implants at an ectopic site within the pelvic cavity (Sampson 1927). However, this theory does not explain the fact that although retrograde menstruation is seen in up to $90 \%$ of women only $10 \%$ of women develop endometriosis (Halme 1984). There is evidence that a variety of environmental, immunological and hormonal factors are associated with endometriosis (Vigano 2004); and genetic loci that confer a risk of endometriosis have been identified (Nyholt 2012). The relative contribution of these and other causal factors needs further elucidation.

Although it is impossible to time the onset of disease, on average women have a 6 - to 12 -year history of symptoms before obtaining a surgical diagnosis of endometriosis, indicative of considerable diagnostic delay (Matsuzaki 2006). Untreated endometriosis is associated with reduced quality of life and contributes to outcomes such as depression, inability to work, sexual dysfunction and missed opportunity for motherhood (Gao 2006).

\section{Treatment of endometriosis}

There is no cure for endometriosis. Treatment options include expectant management, pharmacological (hormonal) therapy and surgery (Johnson and Hummelshoj 2013). Treatment is individualised, taking into consideration the therapeutic goal (pain relief or subfertility) and the location of the disease. Current pharmacological therapies such as the combined oral contraceptive pill, progestogens, weak androgens and $\mathrm{GnRH}$ agonists and antagonists act to reduce the effect of oestrogen on endometrial tissues and suppress menstruation. These drugs can ameliorate the symptoms of dysmenorrhoea and chronic pelvic pain, but are associated with side effects such as breast discomfort, irritability, androgenic symptoms and bone loss. Surgical excision of endometriotic lesions can reduce pain symptoms; however it is associated with high recurrence rates of $40 \%$ to $50 \%$ at 5 years post-surgery (Guo 2009). Early treatment of endometriosis improves pain levels and physical and psychological functioning. Furthermore, improvements in menstrual management (the use of the Mirena coil and the continuous use of the combined contraceptive pill) and fertility preservation (oocyte vitrification) raise the possibility of suppressing the progression of endometriosis and prospectively managing subfertility in endometriosis sufferers. The potential success of these preventative strategies is dependent on an accurate and early diagnosis. A major impediment to earlier and more efficacious treatment of this disease is diagnostic delay due to the invasive nature of standard diagnostic tests (Dmowski 1997).

\section{Diagnosis of endometriosis}

Clinical history and pelvic examination can raise the possibility of a diagnosis of endometriosis, but the heterogeneity in clinical presentation, the high prevalence of asymptomatic endometriosis ( $2 \%$ to $50 \%)$ and the poor association between presenting symptoms and severity of the disease mean that a reliable diagnosis of endometriosis based solely on presenting symptoms is difficult to obtain (Spaczynski 2003; Fauconnier 2005; Ballard 2008). Although an abnormal pelvic examination correlates with the presence of endometriosis on laparoscopy in $70 \%$ to $90 \%$ of cases (Ling 1999), there is a wide differential diagnosis for most positive physical findings. Furthermore, a normal clinical examination does not exclude endometriosis, as laparoscopically proven disease has been diagnosed in more than $50 \%$ women with a clinically normal pelvic examination (Eskenazi 2001). A variety of tests utilising pelvic imaging, blood markers, eutopic endometrium characteristics, urinary markers or peritoneal fluid components have been suggested as diagnostic measures for endometriosis. Although large numbers of the reported markers distinguish women with and without endometriosis in small pilot studies, many do not show convincing potential as a diagnostic test when they are evaluated in larger studies by different research groups. The diagnostic value of these tests has not previously been fully systematically evaluated and summarised using Cochrane methodologies. Currently, there is no simple non-invasive test for the diagnosis of endometriosis that is routinely implemented in clinical practice. 
Surgical diagnostic procedures for endometriosis include laparoscopy (minimal access surgery) or laparotomy (open surgery via an abdominal incision). In the last several decades, laparoscopy has become an increasingly common procedure and has largely replaced traditional open surgery in women suspected of having endometriosis (Yeung 2009). Laparoscopy has significant advantages over laparotomy, creating fewer complications and shorter recovery times. Furthermore a magnified view at laparoscopy allows better visualisation of the peritoneal cavity. Despite continuing controversy in the literature with regard to the superiority of one surgical modality over another in treating pelvic pathology, laparoscopy is the preferred technique to evaluate the pelvis and abdomen and to treat benign conditions such as ovarian endometriomas (Medeiros 2009). Surgery is currently also the only accepted way to determine the extent and severity of endometriosis. Several classification systems have been suggested for endometriosis (Batt 2003; Chapron 2003a; Martin 2006; Adamson 2008), but most researchers and clinicians use the revised American Society for Reproductive Medicine (rASRM) classification, which is internationally accepted as a respected, currently available tool for the objective assessment of the disease (American Society for Reproductive Medicine 1997). The rASRM classification system considers appearance, size and depth of peritoneal or ovarian implants and adhesions visualised during laparoscopy (Table 1) and allows uniform documentation of the extent of disease. Unfortunately this classification system has little value in clinical practice due to the lack of correlation between laparoscopic staging, the severity of symptoms and response to treatment (Vercellini 1996; Guzick 1997; Chapron 2003b).

The European Society of Human Reproduction and Embryology (ESHRE) Special Interest Group for Endometriosis stated in their guidelines for the diagnosis and treatment of endometriosis that for women presenting with symptoms suggestive of endometriosis, a definitive diagnosis of most forms of endometriosis requires visual inspection of the pelvis at laparoscopy as the 'gold standard' investigation (Kennedy 2005). Currently the visual identification of endometriotic tissue in the pelvic cavity during surgery with or without histological confirmation is not just the best available but the only diagnostic test for endometriosis that is used routinely in clinical practice.

The disadvantages of laparoscopic surgery include and are not limited to the high cost, the need for general anaesthesia and the potential for adhesion formation post procedure. Laparoscopy has been associated with a $2 \%$ risk of injury to pelvic organs, a $0.001 \%$ risk of damaging a major blood vessel and a mortality rate of $0.0001 \%$ (Chapron 2003c). Only one third of women who undertake a laparoscopic procedure will receive a diagnosis of endometriosis; therefore many disease-free women are unnecessarily exposed to surgical risk (Frishman 2006).

The validity of laparoscopy as a reference test for endometriosis has been assessed as being highly dependent on the skills of the surgeon. The diagnostic accuracy of laparoscopic visualisation has been compared with histological confirmation in a sole systematic review and is estimated as having a $94 \%$ sensitivity and $79 \%$ specificity (Wykes 2004). Subsequent studies suggested that incorporation of histological verification in the diagnosis of endometriosis may improve diagnostic accuracy (Marchino 2005; Almeida Filho 2008; Stegmann 2008) but these papers have not been systematically reviewed. The clinical significance of histological verification remains debatable, and a diagnosis based on visual findings can be considered reliable with an accurate inspection of the abdominal cavity by properly trained, experienced surgeons (Redwine 2003). Furthermore, excised potential endometriotic tissues are rarely serially sectioned in clinical practice and small lesions can be missed by pathologists in mild disease. Thus sampling inconsistencies are also likely to influence the accuracy of histological reporting.

\section{Summary}

A diagnostic test without the need for surgery would reduce surgical risks, increase accessibility to a diagnostic test and improve treatment outcomes. A need for an accurate and non-invasive diagnostic test for endometriosis continues to encourage extensive research in the field and was endorsed at the international consensus workshop at the 10th World Congress of Endometriosis in 2008 (Rogers 2009). Although multiple markers and imaging techniques have been explored as diagnostic tests for endometriosis, none of them have been implemented routinely in clinical practice and most of them have not been subject to systematic review.

\section{Index test(s)}

This review assesses urinary biomarkers that have been proposed as non-invasive tests for the diagnosis of endometriosis (Table 2), as part of the review series on non-invasive diagnostic tests for endometriosis.

The definition of 'non-invasive' varies between medical dictionaries but refers to a procedure that does not involve penetration of skin or physical entrance to the body (McGraw-Hill Dictionary of Medicine 2006; The Gale Encyclopedia of Medicine 2008). Although bladder catheterization for urine collection is invasive by this definition, when compared to diagnostic surgery for endometriosis, urine tests are generally considered to be 'noninvasive' or 'minimally invasive'. For the purpose of these reviews, we will define all tests that do not involve anaesthesia and surgery as 'non-invasive'.

The advantages of using a urine test for the diagnosis of endometriosis is that it is non-invasive, readily available, and can be self-collected without need for expensive equipment or skilled personnel. It is more acceptable to women, provides a rapid result and is more cost effective when compared to surgery. However urinary testing is dependent on the reliability of laboratory techniques and quality control protocols. Urinary biomarker levels may also be susceptible to variation during the menstrual cycle.

Cellular and molecular processes have been identified that characterise ectopic endometrium and peritoneal fluid in human and animal models (D'Hooghe 2001; Kao 2003; Hull 2008). Markers of these pathophysiological processes have been evaluated in various tissues, including urine, which is increasingly favoured as a fluid for biological testing. Urinary biomarker discovery is a new and rapidly expanding field with most studies published in the last five years. A limited number of endometriosis urinary biomarkers have been evaluated to date and most were assessed in small individual studies. Categories of markers include 1. angiogenesis and growth factors; 2 . cell adhesion molecules and other matrixrelated proteins; 3 . cytokines; 4 . cytoskeleton molecules; 5 . highthroughput molecular markers; 6 . oxidative stress markers; 7 . other 
peptides/proteins shown to influence key events implicated in endometriosis.

A large systematic review of all proposed biomarkers for endometriosis in serum, plasma and urine identified over 100 putative biomarkers, but the authors were unable to identify any biomarker (single or in a panel) that could be recommended for use in clinical practice (May 2010). A more recent narrative review concurred with this conclusion (Fassbender 2015). There is a current need to re-evaluate the diagnostic test accuracy of urine tests for endometriosis using Cochrane methodologies.

\section{Clinical pathway}

Women presenting with symptoms of endometriosis (dysmenorrhoea, dyspareunia, chronic pelvic pain or difficulty conceiving) generally are investigated with a pelvic ultrasound scan to exclude other pathologies, which is in line with international guidelines (Dunselman 2014; SOGC 2010; ACOG 2010). There are no other standard investigative tests and MRI is used conservatively because of its cost. If women seek pain management rather than conception, empirical treatment with progestogens or the combined oral contraceptive pill is commonly started. Diagnostic laparoscopy is considered if empirical treatment fails or if women decline or do not tolerate empirical treatment. In women who have difficulty conceiving, laparoscopy may be undertaken before fertility treatment (particularly if severe pelvic pain or endometrioma are present) or after failed ART (assisted reproductive technology) treatments. Endometriosis can be diagnosed during fertility investigations in women who have minimal or no pain symptomatology.

On average there is a delay of between 6 to 12 years from onset of symptoms to definitive diagnosis at surgery. Early referral to a gynaecologist with the capability to perform diagnostic surgery is associated with a shorter time to diagnosis. Collectively, young women, women in remote and rural locations and women of lower socioeconomic status have reduced access to surgery, and are less likely to obtain a prompt diagnosis of endometriosis.

\section{Prior test(s)}

Most women presenting with symptoms suggestive of endometriosis have a full history and examination and a routine gynaecological ultrasound before a decision is made to have diagnostic surgery. However there is no consensus on whether or not ultrasound or any other test should be routinely used as part of a standardised approach.

\section{Role of index test(s)}

A new diagnostic test can fulfil one of three roles:

1. Replacement: replacing an existing test by having more accuracy, or a similar accuracy with other advantages.

2. Triage: used as an initial step in a diagnostic pathway to identify the group of individuals who need further testing with an existing test. Although ideally a triage test has a high sensitivity and specificity, it may have a lower sensitivity but higher specificity than the current test or vice versa. The triage test does not aim to improve the diagnostic accuracy of the existing test but rather to reduce the number of individuals having an unnecessary diagnostic test.
3. Add-on: used in addition to existing testing to improve diagnostic performance (Bossuyt 2008).

Ideally a diagnostic test is expected to correctly identify all individuals with a disease and to exclude all those without that disease, in other words it should have a sensitivity and specificity of $100 \%$. A high sensitivity indicates that there are a low number of people who have a negative test and do have the disease (i.e. a low number of false-negative results). High specificity corresponds to a low number of people who have a positive test but do not have the disease (i.e. low false-positive results). In practice, however, it is extremely rare to find a test with equally high sensitivity and specificity. An acceptable replacement test would need to have a similar or higher sensitivity and specificity than the current gold standard of laparoscopy. The only systematic review that determines the accuracy of laparoscopy in diagnosing endometriosis reported a sensitivity of $94 \%$, and a specificity of $79 \%$ and we have taken this as a cut off for a replacement test (Wykes 2004).

The purpose of triage tests can vary depending on the clinical context and individuals' priorities. One reasonable approach is to exclude the diagnosis to avoid further unnecessary and expensive diagnostic investigation. High sensitivity tests have few falsenegative results and act to rule conditions out (SnOUT). A negative result from a test with high sensitivity will exclude the disease with high certainty independent of the specificity. As women without disease would be assured of having a negative test, unnecessary invasive interventions can be avoided. However, a positive result has less diagnostic value particularly when the specificity is low. We predetermined that a clinically useful 'SnOUT' triage test should have a sensitivity of $95 \%$ or more and a specificity of $50 \%$ and above. The sensitivity cutoff for a 'SnOUT' triage test was set at $95 \%$ and above, assuming that a $5 \%$ false negative rate is statistically and clinically acceptable. The specificity cutoff was set at $50 \%$ and above, to avoid diagnostic uncertainty in more than $50 \%$ of the population with a positive result.

An alternative approach would be to avoid a missed diagnosis. High specificity tests have few false positive results and act to rule conditions 'in' (SpIN). A positive result for a highly specific triage test indicates a high likelihood of having endometriosis. This information could be used to prioritise these women for surgical treatment. A positive 'SpIN' test could also provide a clinical rationale to start targeted disease-specific medical management in a person without a surgical diagnosis, under the assumption that disease is present. Surgical management could then be reserved for cases when conservative treatment fails. This is particularly relevant in some populations where the therapeutic benefits of surgery for endometriosis have to be carefully balanced with the disadvantages (e.g. young women, women with medical conditions or pain-free women with a history of infertility). In this scenario we considered a sensitivity of $50 \%$ and above and a specificity of $95 \%$ and higher as suitable cutoffs for a 'SpIN' triage test.

We evaluated urine tests for their potential to replace surgery (replacement test) or to improve the selection of women for surgery (triage test) that can either rule out (SnOUT) or rule in (SpIN) the disease. Both types of triage test are clinically useful, minimising the number of unnecessary interventions. Sequential implementation of SnOUT and SpIN tests can also optimise a diagnostic algorithm (Figure 1). We did not assess any test as an add-on test, as we sought tests that reduce the need for surgery 
and not tests that improve the accuracy of the currently available surgical diagnosis.

\section{Figure 1. Sequential approach to non-invasive testing of endometriosis}

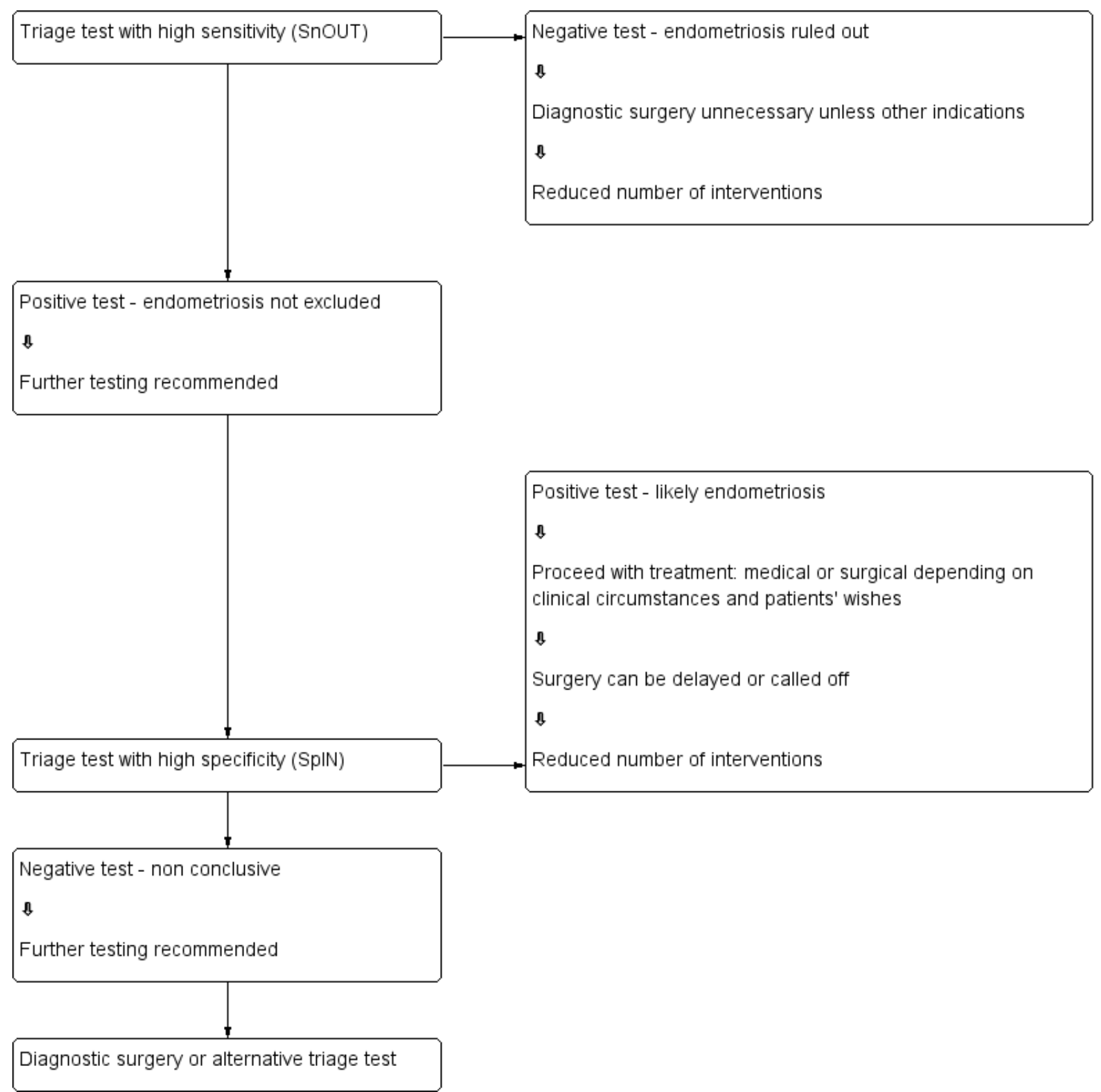

\section{Alternative test(s)}

There are no alternative tests for the diagnosis of endometriosis that are in routine clinical practice.

\section{Rationale}

Many women with endometriosis suffer longstanding pelvic pain and infertility prior to a diagnosis. Surgery is the only current method of diagnosing endometriosis, but it is associated with high costs and surgical risks. A simple and reliable non-invasive test for endometriosis, with the potential to either replace laparoscopy or to triage women in order to reduce surgery, would minimise surgical risk and reduce diagnostic delay. Endometriosis could then be detected at less advanced stages and earlier intervention instituted. This would provide the opportunity for a preventive approach for this debilitating disease. Health care and social security costs of endometriosis would be expected to be reduced by early diagnosis and more cost effective and efficient treatments. Furthermore, identifying urine biomarkers that do not pertain to endometriotic disease would help clinicians and researchers focus on clinically relevant biomarker detection. 


\section{O B JECTIVES}

\section{Primary Objectives}

1. To provide summary estimates of the diagnostic accuracy of urinary biomarkers for the diagnosis of pelvic endometriosis (peritoneal or ovarian or deep infiltrating, or a combination thereof) compared to surgical diagnosis as a reference standard.

2. To assess the diagnostic utility of biomarkers that could differentiate ovarian endometrioma from other ovarian masses.

Urinary biomarkers were evaluated as replacement tests for diagnostic surgery as well as triage tests which would assist decision-making to undertake diagnostic surgery for endometriosis.

\section{Secondary objectives}

1. To investigate the influence of heterogeneity on the diagnostic accuracy of urinary biomarkers for endometriosis. Potential sources of heterogeneity include:

- Characteristics of the study population: age (adolescents vs. later reproductive years); clinical presentation (subfertility, pelvic pain, ovarian mass, asymptomatic women); stage of disease (rASRM classification system); geographic location of study;

- Histological confirmation in conjunction with laparoscopic visualisation compared to laparoscopic visualisation alone;

- Changes in technology over time: year of publication; modifications applied to conventional laboratory techniques;

- Methodological quality: differences in the QUADAS-2 (Quality Assessment of Diagnostic Accuracy Studies-2) evaluation (Table 3), including a) low versus unclear or high risk; b) consecutive versus non-consecutive enrolment; $c$ ) blinding of surgeons to the results of index tests;

- Study design ('single-gate design' vs. 'two-gate design' studies).

2. To assess biomarkers which were not affected by endometriosis and hence were unlikely to discriminate between women with and without the disease.

\section{METHODS}

\section{Criteria for considering studies for this review}

\section{Types of studies}

Published peer-reviewed studies that compared the results of one or several types of urinary biomarkers with the results obtained by surgical diagnosis of endometriosis. Studies were included if they were:

- Randomised controlled trials;

- Observational studies of the following designs:

- 'Single-gate design' (studies with a single set of inclusion criteria defined by clinical presentation). All participants had clinically suspected endometriosis.

- 'Two-gate design' (studies where participants are sampled from distinct populations with respect to clinical presentation). The same study includes participants with a clinical suspicion of having the target condition (e.g. women with pelvic pain) and also participants in whom the target condition is not suspected (e.g. women admitted for tubal ligation). Two-gate studies were eligible only where all cases and controls belonged to the same population in respect to the reference standard (i.e. all the participants were scheduled for laparoscopy) (Rutjes 2005).

- Performed on prospectively collected samples, irrespective of the actual time of the test assay. The timing of sample collection relative to surgery is important because the surgical excision of endometriotic lesions could influence urine biomarker expression and hence bias the results. Therefore, we only included studies where urine was collected before the surgical procedure, i.e. 'prospectively collected'. The studies performed on tissue bank samples collected from prospectively recruited, well-defined populations were considered eligible, which prevented the omission of valuable data from adequately designed studies. The time interval between sample collection and laboratory testing may influence test outcomes which could be dependent on sample storage conditions and the stability of each individual biomarker during storage and freeze/thawing. This information was not readily available for most molecules and was not addressed in this review, but will be considered in future updates if more evidence emerges.

- Performed in any healthcare setting;

- Published in any language;

- We did not impose a minimal limit on the number of participants in the included studies nor the number of studies that have evaluated each index test.

The following studies were excluded:

- Study design:

- Narrative or systematic reviews;

- Studies of retrospective design where the sample collection was performed after execution of reference test;

- Studies of retrospective design where the participants were selected from retrospective review of the case notes/archived samples and information on recruitment methods or study population was not available;

- Case reports or case series;

- Studies reported only in abstract form or in conference proceedings where the full text was not available. This limitation was applied when we faced substantial difficulty in obtaining the information from the abstracts, which precluded a reliable assessment of eligibility and methodological quality.

\section{Participants}

Study participants included reproductive-aged women (puberty to menopause) with suspected endometriosis based on clinical symptoms or pelvic examination, or both, who undertook both the index test and reference standard.

The participants were selected from populations of women undergoing abdominal surgery for the following indications: 1) clinically suspected endometriosis (pelvic pain, infertility, abnormal pelvic examination, or a combination of the above), 2) ovarian mass regardless of symptoms, 3) a mixed group, which consists of women with suspected endometriosis/ovarian mass or women with other benign gynaecological conditions (e.g. surgical sterilisation, fibroid uterus, etc). Asymptomatic women who have an incidental finding of endometriosis at surgery performed for another indication were also included 
Articles that included participants of postmenopausal age were eligible when the data for the reproductive age group was available in isolation. Studies were excluded when the study population involved participants who clearly would not undergo the index test in a clinical scenario or would not benefit from the test (e.g. women with ectopic pregnancies, gynaecological malignancy or acute pelvic inflammatory disease). We also excluded publications where only a subset of participants with a positive index test or reference standard were included in the analysis and the data for the whole cohort were not available.

\section{Index tests}

Any type of urinary biomarker for endometriosis was assessed either separately or in combination with other urine tests. The assessed index tests are presented in Table 2. We included the tests performed in one or several phases of menstrual cycle.

The combined evaluations of urinary biomarkers with other methods for diagnosing endometriosis (e.g. pelvic examination, imaging, blood or endometrial tests) are beyond the scope of this review and are presented separately in another review 'Combined tests for the non-invasive diagnosis of endometriosis'. The studies that solely assessed specific technical aspects, qualitative descriptions of lesion appearance or inter-observer variability of the index tests without reporting the data on diagnostic performance were excluded from the review. When the evaluated biomarker(s) showed differential expression between the groups of women with and without endometriosis, the publication was considered only if the data were reported with sufficient detail for the construction of $2 \times 2$ contingency tables. However, when the contingency tables were not available because the expression level of index test did not significantly differ between the groups and the inclusion criteria were otherwise met, a critical appraisal was undertaken and the study was presented in the descriptive part of the review. Thus the adequately designed studies that identified biomarkers without diagnostic value were evaluated as they provide information that is likely to focus future research on other more clinically useful biomarkers. This methodology also identified biomarkers which were associated with endometriosis in some but not other publications. Evaluations of screening or predictive accuracy tests were not included in this review.

The diagnostic performance of an index test was considered to be high when the test reached the criteria for a replacement test (sensitivity of equal or greater than $94 \%$ with specificity of equal or greater than $79 \%$ ) or triage test (sensitivity of equal or greater than $95 \%$ with specificity of equal or greater than $50 \%$ or vice versa), or approached these criteria (diagnostic estimates within 5\% of the set thresholds). All other diagnostic estimates were considered to be low.

\section{Target conditions}

Pelvic endometriosis, defined as endometrial tissue located in the pelvic cavity: any of the pelvic organs, peritoneum and pouch of Douglas. Three types of pelvic endometriosis were assessed:

1. Peritoneal endometriosis, defined as endometrial deposits detected on peritoneum covering pelvic organs, pelvic side walls or pouch of Douglas;
2. Ovarian endometriosis (endometrioma), defined as an ovarian cyst lined by endometrial tissue and appearing as an ovarian mass of varying size;

3. Deep infiltrating endometriosis (DIE), defined as subperitoneal infiltration of endometrial implants, i.e. when the endometriotic implants penetrate the retroperitoneal space for a distance of $5 \mathrm{~mm}$ or more (Koninckx 1991). DIE may be present in multiple locations, involving either anterior or posterior pelvic compartments, or both.

Certain rare types of endometriosis such as extrapelvic, bladder and ureteric endometriosis were not included in this review because the majority were reported in case reports or case series and laparoscopy or laparotomy are not reliable reference standards for these conditions.

We excluded the studies where diagnosis of endometriosis was not the primary outcome of the trial (e.g. malignant vs benign masses or normal vs. abnormal pelvis) and the separate data for endometriosis were not available.

We also excluded the studies where the findings of the index test formed the basis of selection for the reference standard, because this was likely to distort an assessment of the diagnostic value of index test.

We included studies that recruited selected populations of women with endometriosis (i.e. those with specific rASRM stages), because there is a poor correlation between the rASRM classification and infertility and pain symptoms. Exclusion of these studies could result in a loss of potentially important diagnostic information from otherwise eligible publications. Where possible the impact of these studies was addressed in the assessments of heterogeneity. When a study analysed a large population with a wide spectrum of endometriosis and additionally reported a sub-group analysis of the different stages of disease severity, only estimates for the entire population were considered, because a subgroup analysis does not directly address the review question regarding the clinical utility of the biomarker in detecting the disease.

\section{Reference standards}

The reference standard was visualisation of endometriosis at surgery (laparoscopy or laparotomy) with or without histological confirmation, as this is currently the best available test for endometriosis. Information regarding the inter- and intra-observer correlation of the reference standard was reviewed if reported.

Only studies in which the reference test was performed within 12 months of the urine sample collection were included, on the assumption that disease status could change within a period of one year or longer, either naturally or as a result of treatment. Studies in which the participants did not undergo the reference standard or where the findings of the index test formed the basis of selection for undertaking the reference standard were not included in this review. 


\section{Summary of inclusion/exclusion criteria}

\section{Inclusion criteria:}

- Types of studies:

- Published peer-reviewed;

- RCTs;

- Observational of the following design:

- 'single-gate design' (single set of inclusion criteria defined by clinical presentation): all the participants had clinically suspected endometriosis;

- 'two-gate design' (two sets of inclusion criteria with respect to clinical presentation and one set of inclusion criteria with respect to reference standard): the participants with or without a clinical suspicion of endometriosis scheduled for abdominal surgery;

- Performed on prospectively collected samples, including the tissue bank samples collected from prospectively recruited well-defined population;

- Published in any language;

- Performed in any healthcare setting;

- Any sample size.

- Participants:

- Reproductive-aged women;

- Clinically suspected endometriosis, but included

- women who underwent abdominal surgery for other benign gynaecological conditions and had surgical assessment for presence/absence of endometriosis;

- asymptomatic women who have an incidental finding of endometriosis at surgery performed for another indication;

- Undertook both the index test and reference standard.

- Index tests:

- One or several types of urinary biomarkers;

- Data reported in sufficient detail for the construction of 2 x 2 tables for the tests that showed differential expression between the groups;

- Biomarkers where $2 \times 2$ tables could not be constructed as the results did not differ between women with and without endometriosis, but all other inclusion criteria were met.

- Target condition:

- Pelvic endometriosis

- peritoneal endometriosis;

- ovarian endometrioma;

- DIE;

- combination of the above.

- Reference standard:

- Surgical visualisation of lesions for the diagnosis of endometriosis (laparoscopy or laparotomy) with or without histological verification;

- Performed within 12 months of the urine sample collection.

\section{Exclusion criteria:}

- Types of studies:

- Narrative or systematic reviews;

- Retrospective design where the index test was performed after execution of reference test;

- Prospectively collected samples that were selected from the archived material, but information on the study population or the selection process was unclear;

- Case reports or case series;

- Conference proceedings.

- Participants:

- Included cohort was not representative of the target population that would benefit from the test (e.g. women with known genital tract malignancy, ectopic pregnancies or acute pelvic inflammatory disease);

- Study included participants of postmenopausal age and the data for the reproductive age group were not available in isolation;

- Only participants with positive index test or positive reference standard were included in analysis.

- Index tests:

- Urinary biomarkers presented in combination with other diagnostic tests for endometriosis and separate information for urinary biomarkers was not available;

- Study presented only specific technical aspects of an index test or focused on the biological events, rather than diagnostic performance of the test;

- Study assessed screening or predictive test accuracy.

- Target condition:

- Endometriosis was not the primary outcome of the trial (e.g. malignant vs benign masses or normal vs. abnormal pelvis)

- Atypical, rare sites of endometriosis.

- Reference standard:

- Reference standard performed only in a subset of study/ control group;

- Findings of the index test formed the basis of selection for the reference standard;

- Other than specified in inclusion criteria.

\section{Search methods for identification of studies}

The search strategy was developed in collaboration with the Trials Search Coordinator of the Gynaecology and Fertility Review Group, following recommendations of the Cochrane Handbook for Systematic Reviews of Diagnostic Test Accuracy (de Vet 2008). The searches were not limited to particular types of study design and did not have language or publication date restrictions. The search strategy incorporated words in the title, abstract, text words across the record and the medical subject headings (MeSH). All searches were performed from inception until 31 July 2015. The search strategies for each database and the number of hits per search are presented in Appendix 1; Appendix 2; Appendix 3; Appendix 4. The summary of the results is presented in Results of the search.

\section{Electronic searches}

We searched the following databases to identify the published articles that assessed the diagnostic value of urinary biomarkers for endometriosis: 
- CENTRAL;

- MEDLINE;

- EMBASE;

- CINAHL;

- PsycINFO;

- Web of Science;

- LILACS;

- OAlster;

- TRIP.

- Databases of the trial registers:

- ClinicalTrials.gov;

- World Health Organization (WHO) International Clinical Trials Registry Platform (ICTRP);

- Databases to identify reviews and guidelines as sources of references to potentially relevant studies:

- MEDION;

- DARE;

- PubMed, a 'Systematic Review' search under the 'Clinical Queries' link;

- Searches for papers recently published and not yet indexed in the major databases:

- PubMed (simple search for the last 6 months; the 'related articles' feature was used to locate additional relevant studies).

\section{Searching other resources}

The reference list of all relevant publications (retrieved full texts of the key articles and identified reviews) was handsearched.

An intended attempt to locate the grey literature (unpublished studies and conference proceedings) was abandoned as we faced substantial difficulty in obtaining full-text publications or further details of studies reported in an abstract form.

\section{Data collection and analysis}

\section{Selection of studies}

Two authors of this review (EL, VN) and four authors for the other reviews from this series (Devashana Gupta, Rabia Shaikh, Deepika Arora and Lucy Prentice) scanned the titles of studies identified by our search to remove any clearly irrelevant articles. The titles and abstracts of the remaining studies were reviewed to select potentially relevant publications. The relevant articles were then divided into four categories of endometriosis biomarkers: serum, endometrial, urinary, and combined tests. Two of the urinary biomarker review authors (EL, LH, or VN) independently reviewed each of the full-text versions of the articles selected by title and abstract and assessed them for eligibility for inclusion, based on the criteria listed above under Criteria for considering studies for this review. A single failed eligibility criterion was sufficient for a study to be excluded from the review.

The review authors who assessed the relevance of the studies and eligibility for inclusion were not blinded to the information about each article, including the publishing journal, the names of authors, the institution and the results. Any disagreements were resolved by discussion and, if necessary, in consultation with a third review author (CF), who is an expert in methodological aspects of Cochrane systematic reviews.
When papers updated previous publications and were performed on the same study population at different recruitment points, the most complete data set that superseded previous publications was used to avoid double counting participants or studies. Missing data were retrieved by directly contacting authors to clarify study eligibility. When potentially relevant studies were found in languages other than English, a translation was undertaken. For excluded studies, the reasons for exclusion and details of which criteria were not met were documented. The characteristics of included, excluded and awaiting classification studies are presented under Characteristics of included studies, Characteristics of excluded studies and Characteristics of studies awaiting classification, respectively.

\section{Data extraction and management}

Data were extracted from eligible studies by two independent review authors (EL, LH) and any disagreement was resolved by the third review author (VN). If required, study investigators were contacted to resolve any questions regarding the data.

To collect details from included studies, a data extraction form was specifically designed for this review and pilot tested on three studies of diagnostic accuracy tests for endometriosis. The following information was recorded for each study:

- General information and study design: first author, year of publication, country, language, setting, objectives, inclusion/ exclusion criteria, type of enrolment.

- Characteristics of the study participants: age, symptoms/ history/previous tests, type of target condition and its prevalence in the study population, number of participants enrolled and available for analysis, reasons for withdrawal.

- Features of the index test and reference standard: type, diagnostic criteria, number and experience of the operators, blinding of the operators to other tests or clinical data or both, interobserver variability, time interval between index test and reference standard.

- The reported number of true positives (TP), false negatives (FN), true negatives (TN) and false positives (FP) was used to construct a two-by-two $(2 \times 2)$ table for each index test. If these values were not reported, we attempted to reconstruct the $2 \times 2$ tables from the summary estimates presented in the article.

Data were extracted into Review Manager ${ }^{\circledR}$ (RevMan) software, which was used to graphically display the quality assessment, the diagnostic estimates data and the descriptive analyses.

\section{Assessment of methodological quality}

We used QUADAS-2, a modified version of the QUADAS tool to assess the quality of each included study (Whiting 2011).

The review-specific QUADAS-2 tool and explanatory document are presented in Table 3. Each paper was judged as having a 'low', 'high' or 'unclear' risk for each of four domains and concerns about applicability were assessed in three domains. We considered studies as having low methodological quality when classified at high or unclear risk of bias or at high concern regarding applicability in at least one domain. The assessment of each included study was performed independently by two reviewers (EL, LH, or VN) and disagreements were settled by a third author (CF) or by consensus. Two review authors (EL, LH) independently piloted the topic- 
specific tool to rate four of the included studies with a high level of agreement. Modifications specific to the urinary biomarkers review were made to the signalling questions of the original QUADAS-2 tool and were as following:

1) Domain 1: an original signalling question 'Was a case control design avoided?' was rephrased as 'Was a two-gate design avoided?'. The diagnostic studies are cross-sectional in nature, aiming to compare the result of an index test with the result of the reference standard in same group of participants. In these studies the parameters are measured at a single point of time and the groups are classified by the outcome of the reference standard, albeit the analysis is performed retrospectively. Therefore, unlike epidemiological studies, the terminology 'cohort' and 'case-control' is less informative for diagnostic test trials, and was substituted by 'single-gate' and 'two-gate' designs. This question was included because a two-gate design has more potential to introduce selection bias.

2) Domain 2: an additional signalling question 'Was the phase of the menstrual cycle considered in interpreting the index test?' was introduced to assess bias in the interpretation of the test results. Some biochemical markers are sensitive to fluctuations in steroid sex hormones levels across a menstrual cycle, which could result in the differential expression of endometriosis biomarkers at different cycle phases.

The assessment of methodological quality was undertaken for each domain but a summary score to estimate the overall quality of studies was not calculated (Whiting 2005).

\section{Statistical analysis and data synthesis}

The estimates of sensitivity and specificity were generated in forest plots and plotted in the receiver operating characteristic (ROC) space for each index test using RevMan. The diagnostic performance of each test was investigated and inter study variation in the performance of each index test was visually explored in relation to participant characteristics, study design, and study quality factors. Two or more tests evaluated in the same cohort were included as separate data sets, since the unit of analysis was the test result, not the participant.

For studies that reported subgroup analyses per phase of the menstrual cycle, we presented the data in a clinically relevant way. For instance, pooled estimates were presented when there was no statistically significant difference in biomarker expression between cycle phases. Alternatively, where putative biomarkers demonstrated cycle-dependent expression or were noted to be modulated by ovarian hormones, we reported the test performance either at several time points across the menstrual cycle or in the phase that demonstrated the most distinct difference between groups.

We planned to perform the bivariate logit-normal random effects model for all meta-analyses with four studies or more and a fixed effect meta-analysis of sensitivity and specificity for smaller groups of studies (two to three) in the absence of substantial heterogeneity. When the number of studies was less than four, we did not attempt to estimate the covariance, and reported this as zero. The metaanalyses were performed using SAS NLMIXED software. Results from SAS were input into RevMan to provide plots of the estimated summary points and confidence regions, superimposed on the study-specific estimates of sensitivity and specificity. In this review this aspect of the statistical analysis was unable to be performed due to the paucity of data for each biomarker.

The comparative accuracy of index tests was assessed in two ways. In direct, fully paired comparisons where all the study participants received more than one index test as well as the reference standard, the estimates were plotted in RevMan. If a meta-analysis was possible, test-level covariates in the bivariate logit-normal model were used to identify statistically significant differences. Otherwise the available comparative data were reported in a narrative way and illustrated using forest and ROC plots.

When test performance was judged against the predetermined diagnostic criteria, the point estimates of sensitivity and specificity were considered as the most informative presentation of test performance. We acknowledge that tests with point estimates that did not reach the predetermined criteria but confidence intervals $(\mathrm{Cls})$ which contained values above the threshold, could have diagnostic value. Furthermore tests with point estimates that reached the criteria but $\mathrm{Cls}$ which contained values below the threshold, could have an overestimated diagnostic value. If the range of the Cls rather than the point estimates of the data are used, the predetermined cut-off becomes meaningless. Therefore we did not consider $\mathrm{Cls}$ in qualifying the test performance, but utilised this information in interpreting the reliability of the obtained data.

\section{Dealing with missing data}

Missing data was defined as any information regarding the study population, index tests or reference standard that was not available in the publication which was required to determine the eligibility of the study for inclusion, the methodological quality or to construct the results table. If missing data were identified, the authors were contacted in an attempt to obtain this information. If missing data prevented a clear judgement regarding applicability for inclusion or the construction of accurate $2 \times 2$ tables and the data were not available from the primary investigators (for example we were unable to locate the contact details of the authors or there was no reply from the authors or the authors replied that the requested information was unavailable), the study was excluded from the review.

\section{Investigations of heterogeneity}

Heterogeneity was initially assessed by visually examining the forest plots of sensitivities and specificities and the ROC plots for each index test. The potential sources of heterogeneity are stated in the Secondary objectives. For diagnostic tests where there were more than five eligible studies, we initially planned to formally explore heterogeneity by using study level covariates but we were unable to do so, because of the small numbers of studies in each group. We also planned to assess the sensitivity of results to the inclusion and exclusion of outlying studies in all analyses, but refrained from doing so, again because of the small number of studies for most analyses. It is important to use caution when interpreting small meta-analyses (few studies) with a limited total sample size.

\section{Sensitivity analyses}

We planned to conduct sensitivity analyses to assess the impact of the methodological quality of included studies on the results of any meta-analyses if sufficient data were available. Low quality studies 
were defined by the identification of a high risk of bias for one or more QUADAS-2 domains. We also planned to use the 'leave-oneout' procedure to assess the impact of each study on the metaanalysis results (leading study effect). In the urinary review this was unable to be undertaken due to the paucity of studies evaluating each biomarker.

\section{Assessment of reporting bias}

A comprehensive search of multiple sources for eligible studies, a search of trial registers and no language restrictions minimised the risk of reporting bias. However, publication bias generally arises when studies have a higher chance of being published if their results are positive. Therefore unpublished and published study databases and conference proceedings were initially searched and evaluated. During the process of qualifying the studies for inclusion in this review, we faced substantial difficulty in obtaining fulltext publications or further details of studies published in an abstract form. This precluded a reliable assessment of eligibility and methodological quality and it was decided not to include these publication sources in this review.

\section{RESULTS}

\section{Results of the search}

The literature search identified 33,438 references in the following databases: MEDLINE ( $n=10,328)$, CENTRAL $(n=226)$, EMBASE
( $n=10,313)$, CINAHL $(n=1131)$, PsycINFO $(n=174)$, Web of Science $(n=7425)$, LILACS $(n=420)$, OAlster $(n=446)$, Trip $(n=$ $1648)$, Trial registers for ongoing and registered trials $(n=523)$, MEDION ( $n=2)$, DARE ( $n=99)$, PubMed, a 'Systematic Review' search $(n=418)$, simple search PubMed $(n=267)$. These databases were searched from inception to 20 April - 31 July 2015. The flow of the selection process is presented in Figure 2. Titles were screened to exclude duplicates $(n=9312)$ and clearly irrelevant studies $(n=21,534)$. Another 2575 references were eliminated after reading the abstracts because they did not address the research question or clearly did not meet the inclusion criteria. The full texts of the remaining 16 references were retrieved and assessed for eligibility. Data from two studies required additional clarification from the authors. There were no non-English publications requiring translation. Ultimately, eight studies were eligible according to the inclusion criteria and provided data for the review, six studies were excluded and two studies were defined as awaiting classification. In addition, one ongoing trial was identified through the clinical trials registries (Characteristics of ongoing studies), but the outcomes of this study were not yet available (ongoing, but not recruiting participants). The progress of this study will be monitored and addressed in future updates.

Figure 2. Flow of the studies identified in literature search for systematic review on urinary biomarkers for a noninvasive diagnosis of endometriosis.

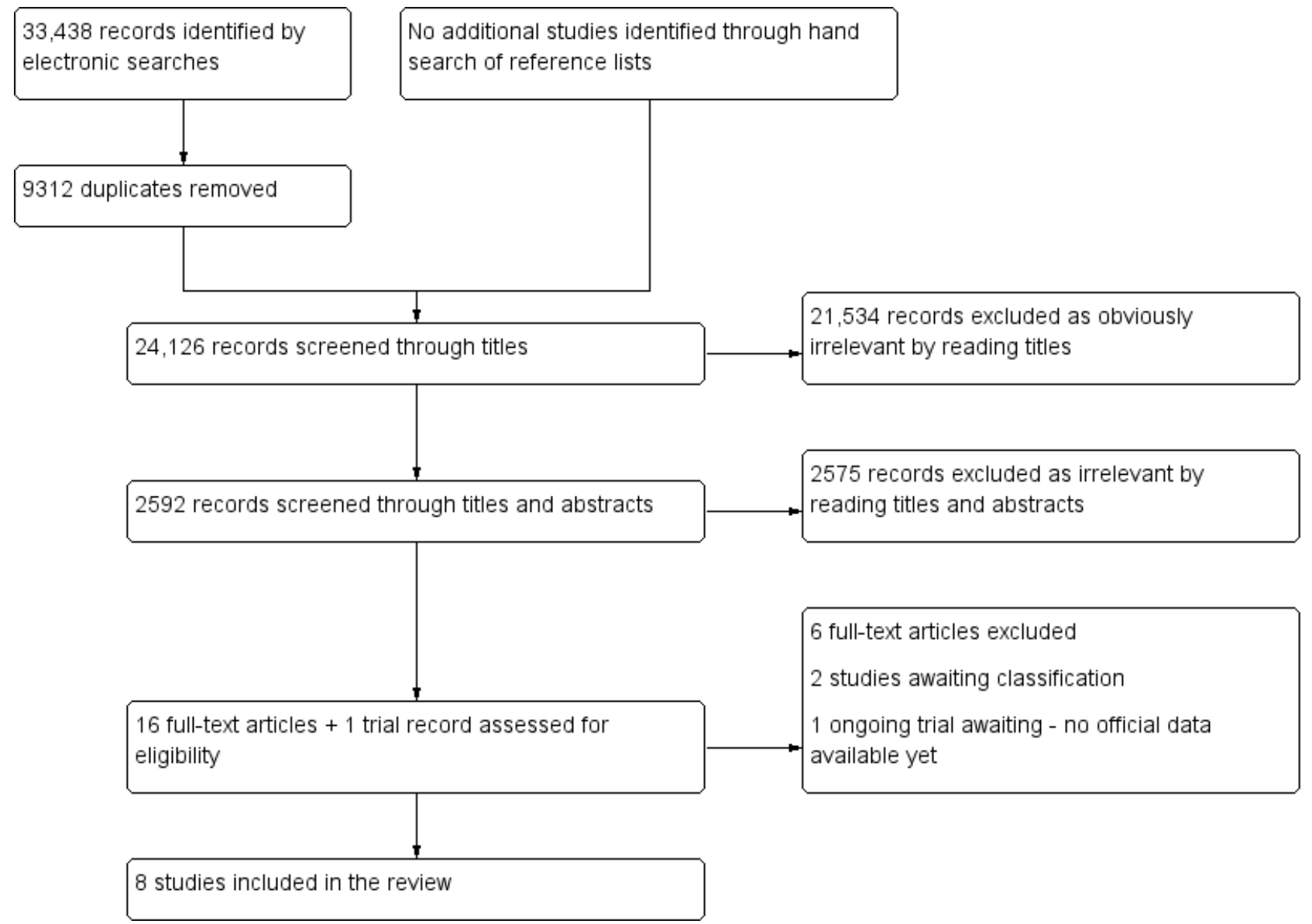




\section{Basic features of the included studies}

The list and details of the included studies are presented in Characteristics of included studies. The eight eligible studies included 646 participants, with a median of 73 women per study (range 39 to 147). Of these studies, five assessed urinary biomarker expression in women with and without endometriosis and included enough data to estimate a diagnostic performance of the investigated test $(n=438$ participants, median 95, range 39 to 147 women). Each study evaluated one or several biomarkers. Most studies reported diagnostic estimates for biomarkers that demonstrated differential expression between women with and without endometriosis, although in one publication this assessment was undertaken for a test that demonstrated no differential expression (Lessey 2014). In three studies there was no difference in the expression between the women with and without endometriosis and the diagnostic test accuracy of the urinary biomarker was not evaluated ( $n=208$ participants, median 70 , range 62 to 76 women). This set of studies was methodologically eligible and the biomarkers identified are unlikely to be of diagnostic utility and hence may not be worth further study.

Four of the included studies were conducted in Asia, two in Europe and two in North America. All the studies were conducted at university hospitals, of which at least three were referral centres for endometriosis. The earliest article was published in 2004, six articles were published after 2010 and four studies were published after 2013. There were no randomised controlled trials and all the studies were observational of cross-sectional design. Five studies were 'single-gate', where both cases and controls were sampled from the same participant population, all of which included women with suspected endometriosis based on clinical presentation (women presenting with pelvic pain, infertility, ovarian mass, or a combination thereof). Three studies were of a 'two-gate design' and included a wider group of participants who were undergoing surgery for various indications. All the included studies assessed women of reproductive age. Laparoscopy was the predominant surgical modality in the included studies, whereas laparotomy was co-utilised in one study. Seven of the included studies used histopathology to confirm the surgical diagnosis. All the included studies evaluated pelvic endometriosis and the reported prevalence of endometriosis varied from $43 \%$ to $66 \%$. Five studies included wide spectrum of endometriosis (rASRM I-IV), two studies included only participants with moderate-severe endometriosis (rASRM stage III-IV) and in one study the information on the severity of the disease was not available. Six studies received financial support, two of which were funded by the pharmaceutical companies, and all the authors declared no conflict of interest. No information was available from the remaining two studies.

\section{Basic features of the excluded studies}

The list and descriptions of the excluded studies are presented in Characteristics of excluded studies. Based on a full text assessment, six publications were excluded, of which one was of retrospective design and the urine samples were collected after the surgical procedure. One study reported statistically significant differences in biomarker levels between the study and control groups, but contained insufficient diagnostic accuracy information for the construction of $2 \times 2$ contingency tables. One excluded paper presented the qualitative evaluation of urinary biomarkers and did not define a specific test for diagnostic assessment. A further three studies were excluded as they evaluated urinary excretion of the environmental toxins and their association with risk of endometriosis. For two studies there was insufficient data to confirm eligibility and these were classified as awaiting classification at the time of publication. These studies are outlined in Characteristics of studies awaiting classification and will be addressed in future updates of this review.

\section{Methodological quality of included studies}

The quality of the included studies is illustrated in the QUADAS-2 results summary (Figure 3 and Figure 4). Overall, the studies were of poor methodological quality and all studies had an unclear or high risk of bias in at least one domain.

Figure 3. Risk of bias and applicability concerns graph: review authors' judgements about each domain presented as percentages across included studies

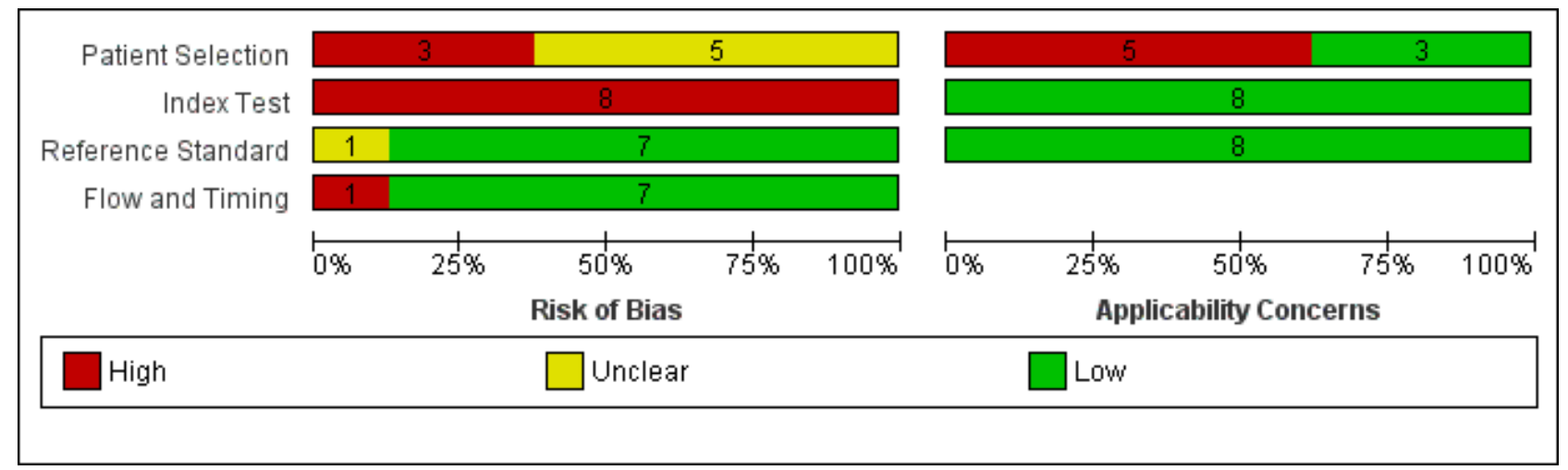


Figure 4. Risk of bias and applicability concerns summary: review authors' judgements about each domain for each included study

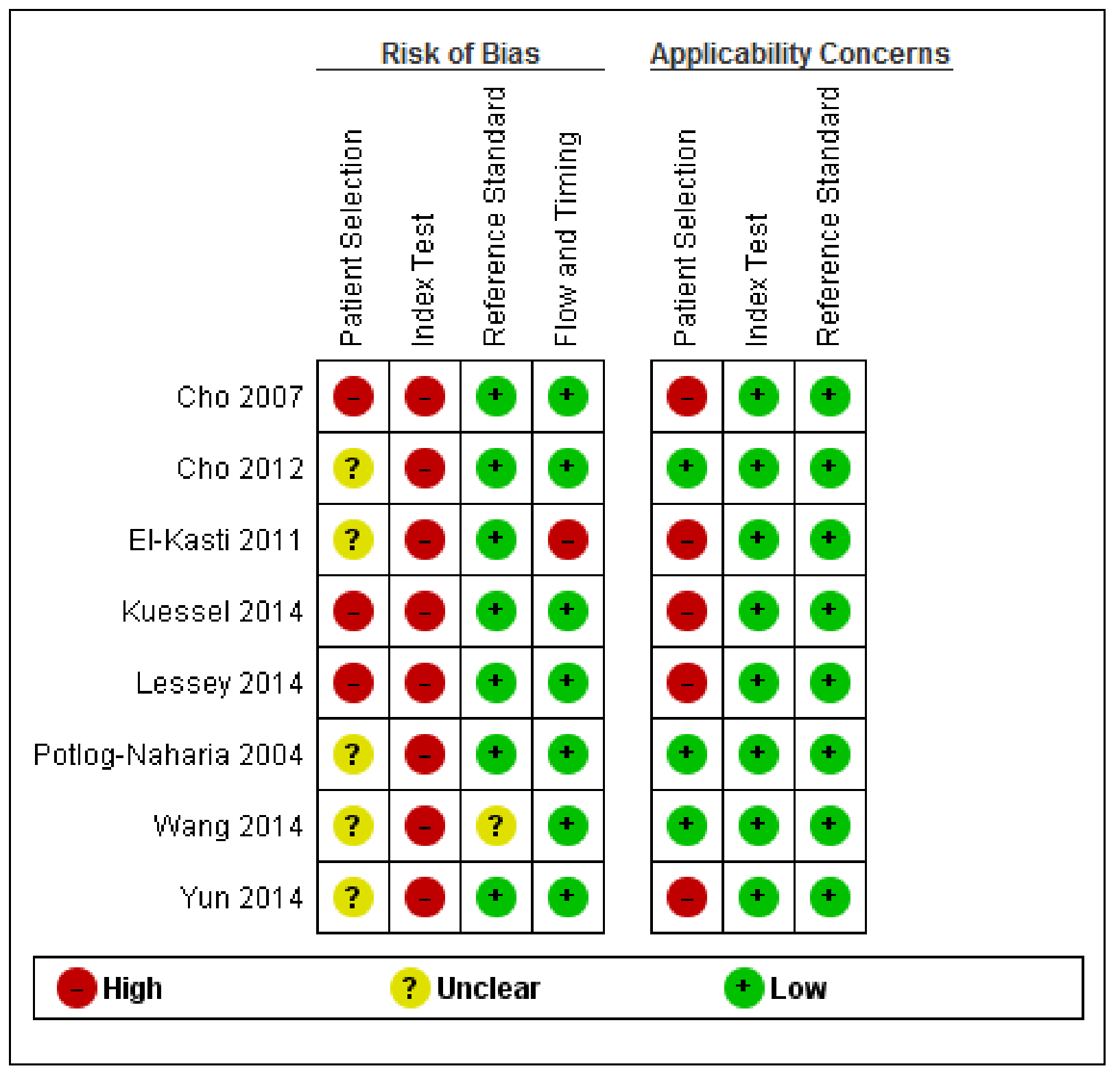

No studies presented a low risk of participant selection bias; five studies demonstrated an unclear risk (Potlog-Naharia 2004; ElKasti 2011; Cho 2012; Wang 2014; Yun 2014); and three studies were assessed at high risk for this domain (Cho 2007; Kuessel 2014; Lessey 2014). Non-consecutive or non-random participant selection, utilisation of a two-gate design for participant selection and the absence of a clear definition of inclusion/exclusion criteria were the main reasons for a 'high risk' assessment of bias.

All the studies demonstrated a high risk of index test interpretation bias (Potlog-Naharia 2004; Cho 2007; El-Kasti 2011; Cho 2012; Kuessel 2014; Lessey 2014; Wang 2014; Yun 2014). A lack of clear prespecified criteria for a positive diagnosis and index test operators not being blind to the results of reference standard were the main reasons for a 'high risk' assessment. A high risk of bias for this domain was also attributed to the articles where the phase of menstrual cycle was not considered in interpreting the index test.
This was considered an important criterion, since varying ovarian hormones across the cycle could influence biomarker expression and undermine the reliability of the results. Furthermore, the skill level of a test operator and interobserver variability, both of which directly affect performance of the tests, were rarely reported. As the criteria for a positive index test were variable between the studies and the index test protocols were not standardised, quality judgements for the index test were complex.

Seven studies were at low risk of bias in the 'reference standard' domain (Potlog-Naharia 2004; Cho 2007; El-Kasti 2011; Cho 2012; Kuessel 2014; Lessey 2014; Yun 2014); one study was classified at unclear risk (Wang 2014); and no studies demonstrated a high risk. An unclear risk of bias was assigned if there was not enough information to determine how likely the reference standard was to have correctly classified the target condition. Specifically, surgical procedures were not well described, the criteria for a positive 
reference standard were not stated, it was unclear if histology was utilised to confirm surgical diagnosis, or there was no information regarding the experience of the surgeons or the pathologists (or both) involved.

Seven studies presented a low risk of bias in the 'flow and timing' domain (Potlog-Naharia 2004; Cho 2007; Cho 2012; Kuessel 2014; Lessey 2014; Wang 2014; Yun 2014); no studies demonstrated an unclear risk; and one study carried a high risk (El-Kasti 2011). In every study all participants received the same reference standard. The time interval between the index test and the reference standard was placed as 12 months or less and the most commonly reported time interval was immediately before surgery. A high risk of bias was assigned if there were unexplained withdrawals that exceeded $5 \%$ of the enrolled population or if the reason for withdrawal could introduce selection bias regarding the samples analysed.

Three studies presented a low concern for participant selection applicability (Potlog-Naharia 2004; Cho 2012; Wang 2014); and five were of high concern (Cho 2007; El-Kasti 2011; Kuessel 2014; Lessey 2014; Yun 2014). A high concern in participant selection applicability was assigned if the study utilised two-gate selection for cases and controls or if only a limited spectrum of disease was evaluated. Additional uncertainty regarding the accuracy of the index test in the entire clinically relevant population is introduced if the urine biomarker varied across participant subgroups. In our view, any sampling deviation from a representative group of the entire clinically relevant population could skew the estimates of diagnostic accuracy in any direction.

All the studies presented a low concern of index test applicability, presenting sufficient information to conclude that the index test, its conduct or interpretation matched the review question (Potlog-
Naharia 2004; Cho 2007; El-Kasti 2011; Cho 2012; Kuessel 2014; Lessey 2014; Wang 2014; Yun 2014).

All eight studies were of low concern for applicability in regards to the reference standard and none had a high or unclear concern (Potlog-Naharia 2004; Cho 2007; El-Kasti 2011; Cho 2012; Kuessel 2014; Lessey 2014; Wang 2014; Yun 2014). All the included studies implemented pelvic surgery (laparoscopy or laparotomy) as a reference standard, which could be relied upon to match the review question.

\section{Findings}

A total of six urinary biomarkers were evaluated in the eight included studies, of which four biomarkers had a diagnostic evaluation in five studies (Summary of findings 1). Three biomarkers were not altered by the presence of endometriosis and were evaluated in three other studies (Summary of findings 2).

\section{1) Enolase 1 (NNE)}

The diagnostic performance of urinary NNE was evaluated in one study (59 women, follicular or luteal cycle phase, only moderate to severe endometriosis, rASRM III-IV) (Yun 2014). Urinary NNE expression was not influenced by cycle phase and was significantly greater $(P=0.026)$ in women with endometriosis when corrected for creatine excretion (NNE-Cr). Using a cut-off threshold of more than $0.96 \mathrm{ng} / \mathrm{mgCr}$, the sensitivity was $0.56(95 \% \mathrm{Cl}, 0.40$ to 0.72$)$, and the specificity $0.70(95 \% \mathrm{Cl}, 0.46$ to 0.88$)$ and did not meet the criteria for either replacement or triage tests (Figure 5). Further testing in larger studies including participants with a wider spectrum of endometriosis is needed to confirm the role of NNE in detecting endometriosis. 
Figure 5. Summary ROC Plot of NNE-Cr for detection of endometriosis utilising a cut-off $>0.96 \mathrm{ng} / \mathrm{mgCr}$. Each point represents the pair of sensitivity and specificity for evaluation. The bars correspond to $95 \% \mathrm{Cls}$.

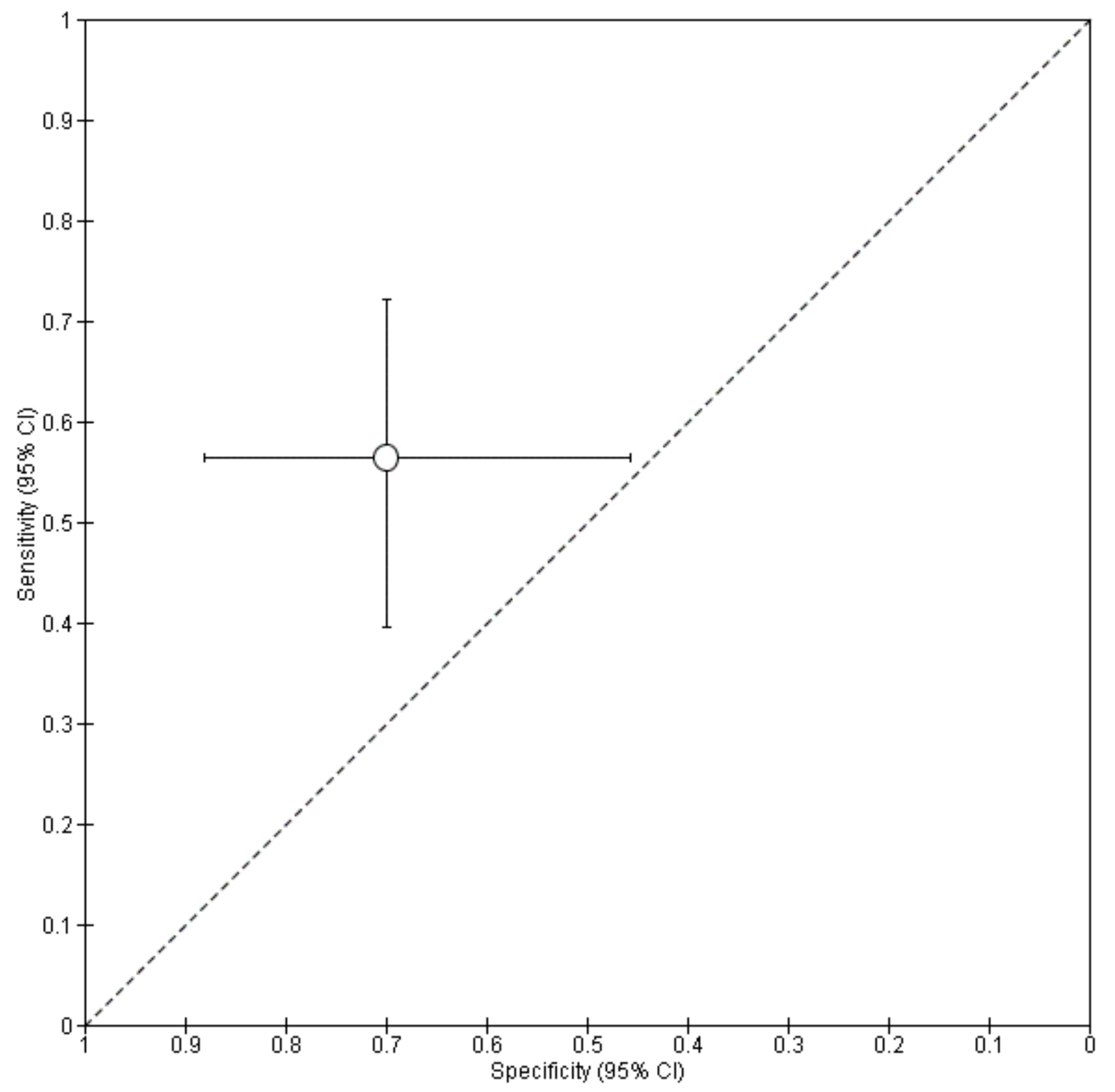

\section{2) Vitamin D-binding protein (VDBP)}

The diagnostic performance of urinary VDBP was evaluated in one study only, which included 95 women in the follicular or luteal cycle phase (Cho 2012). Even though the study included endometriosis of varying severity (rASRM I-IV), more than $90 \%$ of women with endometriosis had moderate to severe disease (52/57). Urinary VDBP levels corrected for creatinine (VDBP-Cr) expression were significantly greater in participants with endometriosis $(P=0.001)$. However, VDBP-Cr only distinguished women with and without endometriosis in the luteal phase $(P=0.042)$ of the cycle. The cutoff value of more than $87.83 \mathrm{ng} / \mathrm{mgCr}$ demonstrated a sensitivity of $0.58(95 \% \mathrm{Cl}, 0.44$ to 0.71$)$ and a specificity of $0.55(95 \% \mathrm{Cl}, 0.38$ to 0.71 ) (Figure 6). The results are discouraging, but further evaluation of VDBP across the spectrum of endometriosis particularly in the luteal phase may help to clarify its diagnostic role in endometriosis. 
Figure 6. Summary ROC plot of VDBP-Cr for detection of endometriosis utilising a cut-off $>87.83 \mathrm{ng} / \mathrm{mgCr}$. Each point represents the pair of sensitivity and specificity for the evaluation. The bars correspond to $95 \% \mathrm{Cls}$.

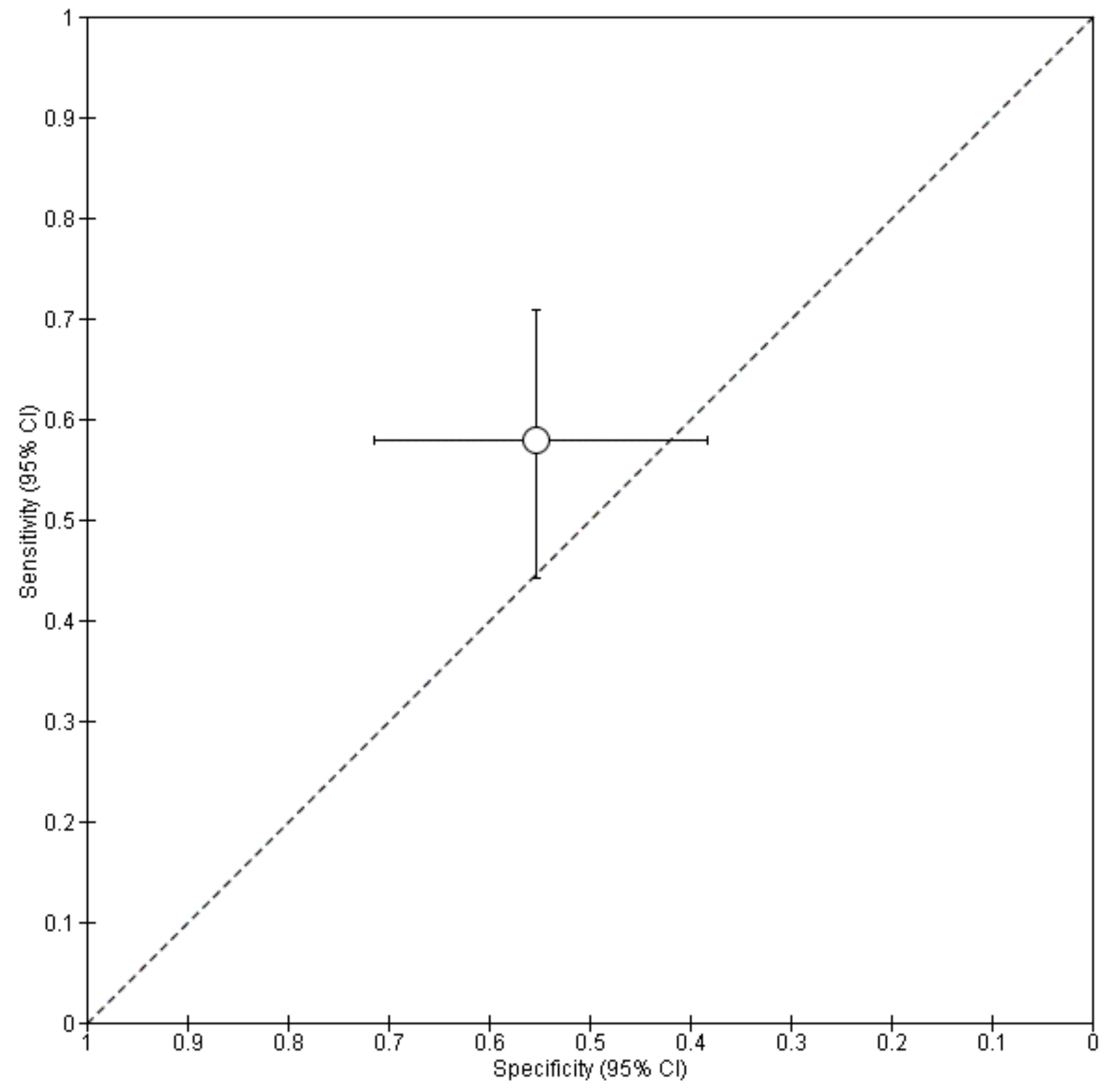

\section{3) MALDI-TOF-MS proteomics}

Two studies, including seven data sets comprising 186 women, assessed the accuracy of proteomic techniques in detecting endometriosis (Figure 7; Figure 8). One study included 39 women in the follicular, peri-ovulatory or luteal phases (El-Kasti 2011). Six significant putative peptide markers distinguished controls from women with moderate to severe endometriosis (rASRM III-IV), four of these in the peri-ovulatory phase and two in the luteal phase. The diagnostic accuracy of two peptides only identified by their mass profile were evaluated. The peri-ovulatory peptide mass of 1767.1
Da using a cut-off of 35.22 or more arbitrary units (a.u.) showed a sensitivity of $0.75(95 \% \mathrm{Cl}, 0.43$ to 0.95$)$ and a specificity of 0.87 (95\% $\mathrm{Cl} 0.60$ to 0.98 ). The luteal peptide mass of $1824.3 \mathrm{Da}$ using a cutoff of 29.34 or more a.u. showed a sensitivity of $0.77(95 \% \mathrm{Cl}, 0.46$ to $0.95)$ and a specificity of $0.73(95 \% \mathrm{Cl}, 0.45$ to 0.92$)$. The diagnostic performance of the other four peptides were not assessed. It was noted that 14 participants with minimal to mild endometriosis were excluded from this analysis. None of the biomarkers met the criteria for a replacement or triage test, but this observation provides too little data to draw conclusions regarding the diagnostic role of these urinary peptides in endometriosis. 
Figure 7. Summary ROC plot of Proteome for detection of endometriosis. Each point represents the pair of sensitivity and specificity for each evaluation. The size of each point is proportional to the sample size and the shape designates the tests including different proteins. The bars correspond to $95 \% \mathrm{Cls}$ of each individual evaluation. The data were not assessed by meta-analysis.

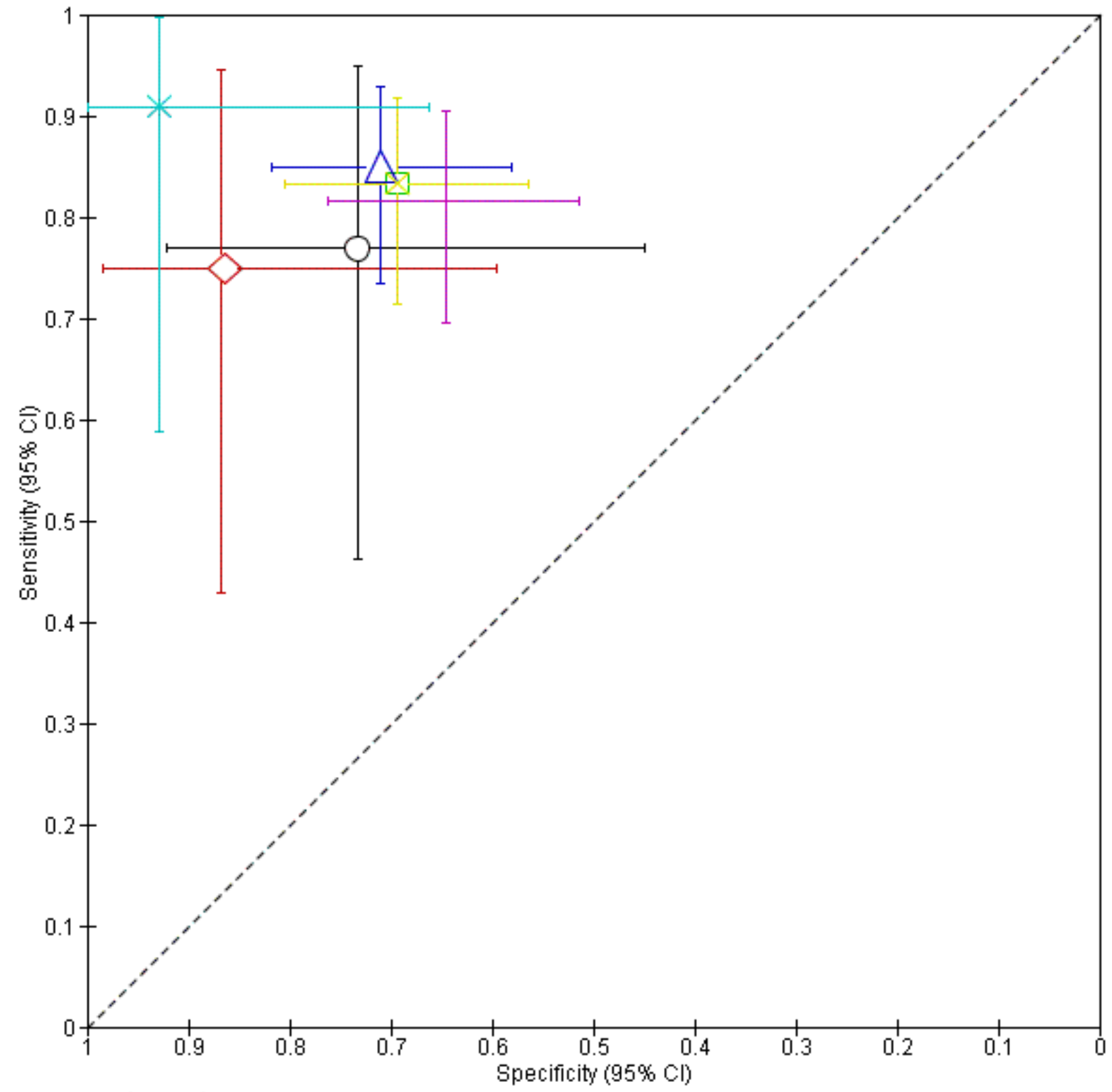

- Legend

O Proteome by MALDI-TOF-MS (peptide $\mathrm{m} / \mathrm{z} 1824.3 \mathrm{Da} ; \geq 29.34 \mathrm{au}$ )

$\triangle$ Proteome by MALDI-TOF-MS (peptide $\mathrm{m} / 21767.1 \mathrm{Da} ; \geq 35.22 \mathrm{au}$ )

$\square$ Proteome by MALDI-TOF-MS (peptide m/z 2052.3 Da; cut-off not reported)

$\triangle$ Proteome by MALDI-TOF-MS (peptide m/z 3393.9 Da; cut-off not reported)

Proteome by MALDI-TOF-MS (peptide $\mathrm{m} / \mathrm{z} 1579.2 \mathrm{Da}$ [collagen alpha 6 (IV) chain precursor];...

+ Proteome by MALDI-TOF-MS (peptide $\mathrm{m} / 2891.6 \mathrm{Da}$ [collagen alpha1 chain precursor].....

* Proteome by MALDI-TOF-MS (5 peptides m/z $1433.9+1599.4+2085.6+6798.0+3217.2 \ldots$ 
Figure 8. Forest plot of proteome for detection of endometriosis. Plot shows study-specific estimates of sensitivity and specificity (squares) with $95 \% \mathrm{Cl}$ (black line), country in which the study was conducted, menstrual cycle phase at which the test was performed and severity of the disease assessed by each study, reported as rASRM stage. FN: false negative; FP: false positive; TN: true negative; TP: true positive.

Proteome by MALDI-TOF-MS (peptide m/z 1824.3 Da; $\geq 29.34$ au)

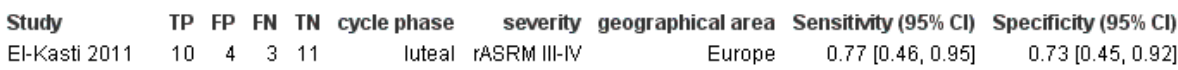

Proteome by MALDI-TOF-MS (peptide m/z 1767.1 Da; $\geq 35.22$ au)

Sensitivity $(95 \% \mathrm{Cl}) \quad$ Specificity $(95 \% \mathrm{Cl})$

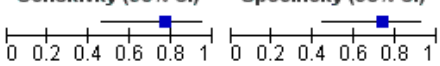

Study TP FP FN TN cycle phase severity geographical area Sensitivity $(95 \% \mathrm{Cl})$ Specificity $(95 \% \mathrm{Cl})$

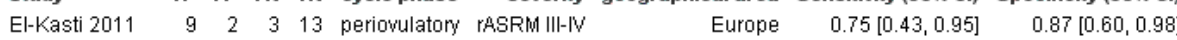

Sensitivity $(95 \% \mathrm{Cl}) \quad$ Specificity $(95 \% \mathrm{Cl})$

Proteome by MALDI-TOF-MS (peptide m/z 2052.3 Da; cut-off not reported)

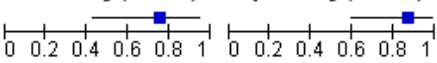

Study TP FP FN TN cycle phase severity geographical area Sensitivity $(\mathbf{9 5} \% \mathrm{Cl})$ Specificity $(\mathbf{9 5} \% \mathrm{Cl}) \quad$ Sensitivity $(\mathbf{9 5} \% \mathrm{Cl}) \quad$ Specificity $(\mathbf{9 5} \% \mathrm{Cl})$

Wang $2014 \quad 50 \quad 19 \quad 10 \quad 43$ follicular or luteal rASRM I-IV $\quad$ Asia $0.83[0.71,0.92] \quad 0.69[0.56,0.80]$

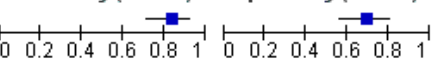

Proteome by MALDI-TOF-MS (peptide m/z 3393.9 Da; cut-off not reported)

Study TP FP FN TN cycle phase severity geographical area Sensitivity $(\mathbf{9 5} \% \mathrm{Cl})$ Specificity $(\mathbf{9 5} \% \mathrm{Cl}) \quad$ Sensitivity $(\mathbf{9 5} \% \mathrm{Cl}) \quad$ Specificity $(\mathbf{9 5} \% \mathrm{Cl})$ $\begin{array}{lllllllll}\text { Wang } 2014 & 51 & 18 & 9 & 44 & \text { follicular or luteal rASRM I-IV } & \text { Asia } & 0.85[0.73,0.93] & 0.71 \\ {[0.58,0.82]}\end{array}$

Proteome by MALDI-TOF-MS (peptide m/z 1579.2 Da [collagen alpha 6(IV) chain precursor]; cut-off not reported)

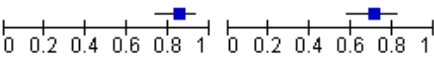

Study TP FP FN TN cycle phase severity geographical area Sensitivity $(95 \% \mathrm{Cl})$ Specificity $(95 \% \mathrm{Cl}) \quad$ Sensitivity $(95 \% \mathrm{Cl}) \quad$ Specificity $(95 \% \mathrm{Cl})$ Wang $2014 \quad 50 \quad 19 \quad 10 \quad 43$ follicular or luteal rASRM I-IV $\quad$ Asia $0.83[0.71,0.92] \quad 0.69[0.56,0.80]$

Proteome by MALDI-TOF-MS (peptide m/Z 891.6 Da [collagen alpha1 chain precursor];; cut-off not reported)

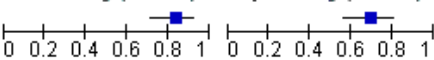

Study TP FP FN TN cycle phase severity geographical area Sensitivity (95\% Cl) Specificity $(\mathbf{9 5} \% \mathrm{Cl}) \quad$ Sensitivity $(\mathbf{9 5} \% \mathrm{Cl}) \quad$ Specificity $(\mathbf{9 5} \% \mathrm{Cl})$

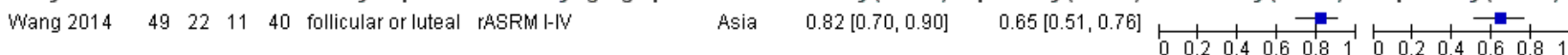

Proteome by MALDI-TOF-MS (5 peptides m/z 1433.9 +1599.4 + 2085.6 + 6798.0 + 3217.2 Da; cut-off not reported)

Study TP FP FN TN cycle phase severity geographical area Sensitivity $(\mathbf{9 5} \% \mathrm{Cl})$ Specificity $(\mathbf{9 5} \% \mathrm{Cl}) \quad$ Sensitivity $(\mathbf{9 5} \% \mathrm{Cl}) \quad$ Specificity $(\mathbf{9 5} \% \mathrm{Cl})$

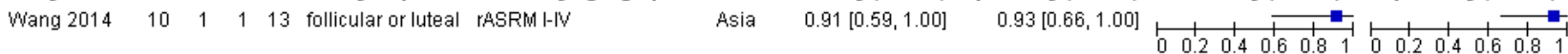

Another study (122 women, follicular or luteal cycle phase, rASRM I-IV, unclear histological confirmation) identified 36 peptides that were significantly different between the endometriosis and the control groups (Wang 2014). The peptide pattern did not vary between follicular and luteal phases and four peptides, that were down-regulated in endometriosis, were further evaluated for diagnostic performance. The 2052.3 Da mass peptide demonstrated a sensitivity of $0.83(95 \% \mathrm{Cl}, 0.71$ to 0.92$)$ and a specificity of $0.69(95 \% \mathrm{Cl}, 0.56$ to 0.80$)$, and the 3393.9 Da mass peptide had a sensitivity of $0.85(95 \% \mathrm{Cl}, 0.73$ to 0.93$)$ and a specificity of $0.71(95 \% \mathrm{Cl}, 0.58$ to 0.82$)$. The other two peptides were able to be identified by their mass spectra and were a collagen alpha-6(IV) chain precursor fragment (1579.2 Da) with a sensitivity of $0.83(95 \% \mathrm{Cl}, 0.71$ to 0.92$)$ and a specificity of $0.69(95 \% \mathrm{Cl}$, 0.56 to 0.80 ) and a type VIII, IX, XV collagen alpha1 chain precursor fragment $(891.6 \mathrm{Da})$ with a sensitivity of $0.82(95 \% \mathrm{Cl}, 0.70$ to 0.90$)$ and a specificity of $65(95 \% \mathrm{Cl}, 0.51$ to 0.76$)$.The cut-off thresholds were not reported for any of these analyses. Three algorithms were developed using peptide peak clusters in diagnostic models, (the Genetic algorithm (GA), the decision tree algorithm (DTA), and the quick classifier algorithm (QC)). The GA algorithm showed the highest diagnostic estimates comprising five peptides of $1433.9 \mathrm{Da}$, 1599.4 Da, 2085.6 Da, 6798.0 Da, and 3217.2 Da and was further validated in a blinded test group analysis of 25 randomly selected participants, 11 of which had endometriosis. The estimates of the validation test showed a high sensitivity of $0.91(95 \% \mathrm{Cl}, 0.59$ to $1.00)$ and a high specificity of $0.93(95 \% \mathrm{Cl}, 0.66$ to 1.00$)$, which approach the criteria for the replacement of SnOUT and SpIN triage tests. These results require further validation in large, independent, well-defined populations, displaying a wide spectrum of disease, using standardised and reproducible methodologies.

\section{4) Cytokeratin-19 fragments (uCYFRA 21-1)}

Two studies that included 174 women assessed the performance of Cytokeratin 19 (CK 19) as a biomarker in detecting endometriosis by measuring urine fragment UCYFRA 21-1. Both studies concluded that CK 19 was not altered by the presence of endometriosis and that their levels were not affected by menstrual cycle phases (Kuessel 2014), by severity of the disease or when the levels were normalised to urine creatinine or urine protein (Lessey 2014). Only one of these studies (98 participants, cycle phase not reported, rASRM I-IV) evaluated the diagnostic accuracy of UCYFRA 21-1 (Lessey 2014), demonstrating a very low sensitivity of $0.11(95 \%$ $\mathrm{Cl}, 0.05$ to 0.22$)$ with a high specificity $0.94(95 \% \mathrm{Cl}, 0.81$ to 0.99$)$, using a chosen cut-off of more than $5.3 \mathrm{ng} / \mathrm{ml}$ (Figure 9). This evidence suggests that the cytokeratin 19 molecule is not reliable as a diagnostic test for endometriosis, but further testing is required to confirm or refute these findings. 
Figure 9. Summary ROC plot of CK 19 for detection of endometriosis utilising a cut-off $>5.3 \mathrm{ng} / \mathrm{ml}$. Each point represents the pair of sensitivity and specificity for the evaluation. The bars correspond to $95 \% \mathrm{Cls}$.

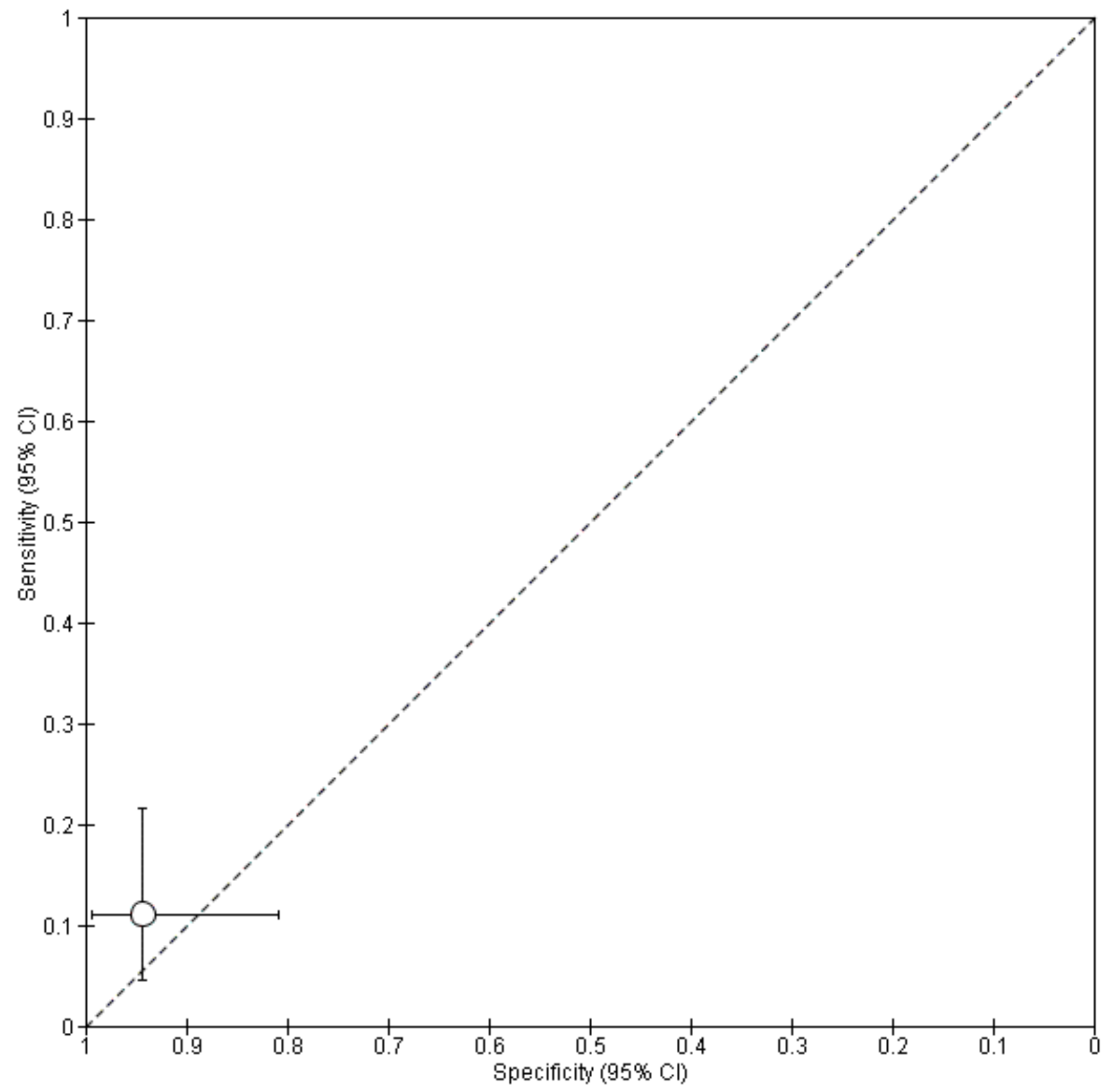

5) Vascular endothelial growth factor (VEGF) or vascular endothelial growth factor-A (VEGF-A)

Two studies in 132 women assessed the performance of urinary VEGF in diagnosing endometriosis (Potlog-Naharia 2004; Cho 2007). The levels were corrected to urinary creatinine in both studies, and one study showed no differences in excretion across the menstrual cycle (Potlog-Naharia 2004). There was no significant difference between the control and endometriosis groups seen in either study, and the diagnostic accuracy was not evaluated.

\section{6) Tumour necrosis factor-alpha (TNF-alpha)}

Urinary TNF-alpha levels were not significantly different in one study (70 participants, follicular or luteal cycle phase, rASRM I-IV) and the diagnostic accuracy was not evaluated (Cho 2007).

\section{Investigations of heterogeneity and sensitivity analyses}

The potential sources of heterogeneity are outlined in Secondary objectives. Although we attempted to assess these sources of heterogeneity, there were not enough studies evaluating each test to make this a meaningful analysis. Furthermore, the sensitivity analyses were not possible due to the small number of studies. 


\section{DISCUSSION}

\section{Summary of main results}

Only a few urinary biomarkers have been assessed in small numbers of individual studies providing insufficient data to perform a meta-analysis. No urinary test met the criteria of either replacement or triage test for detecting endometriosis. The GA algorithm, a combined test of five urinary peptides of $1433.9 \mathrm{Da}, 1599.4 \mathrm{Da}, 2085.6 \mathrm{Da}, 6798.0 \mathrm{Da}$, and 3217.2 Da demonstrated the highest diagnostic estimates for detecting endometriosis, which approached but did not meet the criteria for the replacement of both the SnOUT and SpIN triage tests (Wang 2014). The algorithm was validated in an independent test group but, as this test was only reported in one study, meaningful conclusions regarding its value in clinical practice cannot be drawn. Certain urinary peptides identified through high-throughput MALDI-TOF-MS method showed potential in detecting endometriosis. However, urinary proteome studies showed considerable heterogeneity with respect to the population studied, the way the samples were processed and the data analysis. The molecular masses of the identified differentially regulated peptides were entirely inconsistent across studies, with most remaining unidentified biologically. Establishing standardised analytical processes, consistent sets of markers and defined cut-off thresholds would improve the assessment of urinary peptides as a diagnostic tool for endometriosis and further large-scale studies are required before meaningful conclusions can be made.

CK 19 and VEGF were found not associated with endometriosis in more than one study, indicating that these biomarkers are unlikely to have diagnostic value. In view of the paucity of data, further large studies are still needed to support this statement.

There were no studies that assessed the role of urinary biomarkers in the diagnosis of ovarian endometrioma.

\section{Strengths and weaknesses of the review}

This review is part of a comprehensive review series of minimally invasive biomarkers for the diagnosis of endometriosis. A very thorough search of the current literature was undertaken and included studies written in languages other than English. Two independent reviewers extracted the data and used a modified QUADAS-2 tooL to perform quality assessments. Stringent selection criteria ensured that eligible studies utilised prospectively collected samples and only included women of reproductive age, which minimised the risk of bias in interpreting the reference standard and index test. An additional strength of this review was that the authors of the studies were approached in an attempt to obtain any missing information required to assess eligibility and critically appraise the studies. The inclusion of studies demonstrating that biomarker levels did not significantly differ in endometriosis introduced an additional dimension to the interpretation of the results, particularly for the biomarkers with contradictory results. Furthermore, biomarkers which were consistently reported as unchanged by the disease could be excluded from the list of putative biomarkers for endometriosis. Although this has little influence on the conclusions of this review due to the paucity of the available data, the relevance of this method will increase in future updates that describe this growing body of evidence.
The main limitation of this review is that there are only individual small studies for all the evaluated index tests. A meaningful metaanalysis of index test performance was not possible for any urinary biomarker. There was variation between studies with respect to the included populations, the severity of endometriosis, when in the menstrual cycle phase sampling was performed and whether the urinary biomarker levels were corrected against creatinine excretion. Also, most of the included studies determined the diagnostic cut-off thresholds using a ROC analysis without any subsequent validation in an independent cohort. Lack of validation of the diagnostic data in conjunction with the low number of studies for the majority of the presented tests contributed to the low quality of evidence presented in this review. We now have available a standardised methodology for fluid bio specimen collection, processing and storage and we recommend adhering to these standards in future diagnostic studies (Rahimoglu 2014).

Another weakness is the variation in the selection of the case and control groups with inclusion of participants that may not reflect a clinically representative population. The reported prevalence of endometriosis in most studies was generally higher (43\% to 66\%) than previously reported prevalences of endometriosis (6\%-10\% in the general female population and $35 \%-50 \%$ in symptomatic women) (Giudice 2004). This may reflect a high level of surgical diagnostic expertise but could be due to pre-selection of more challenging cases in tertiary referral centres and there is a high risk of participant selection bias in most of the studies. Selection bias appeared to be reduced but not eliminated by consecutively enrolling participants; however the information on method of enrolment was missing in most of the included studies. More than a third of the included studies $(3 / 8,38 \%)$ were of a 'twogate design' and included a wide group of participants who underwent surgery for various indications. Inclusion of healthy asymptomatic individuals or participants with other pathological conditions represents potential selection bias with regard to the control group which could have biased the test outcomes. Thirtyeight percent of the studies included either women with a limited spectrum of endometriosis $(n=2)$ or did not provide information on the severity of target condition $(n=1)$. These studies were included to avoid omission of potentially valuable diagnostic information, but each of the above factors could skew the diagnostic estimates in either direction and subsequently interfere with the interpretation of the index test results. It was not possible to evaluate population and disease spectrum effects on the data because there were so few single reports for all the urinary biomarkers.

Inappropriate assignation to the endometriosis and control groups could not be excluded in some studies and is another weakness of the review. Surgical misdiagnosis is a potential cause of bias as the number and experience of the surgical team, the surgical diagnostic criteria and the surgical methods were poorly described in most of the included studies. We now have a standardised technique for performing laparoscopy and we recommend that any future studies use this standardised method of undertaking laparoscopy (Becker 2014). Additionally, we did not confine the studies included in this review to those that reported histological confirmation of endometriotic lesions. Although a recent ESHRE guideline stated that evidence is lacking to support laparoscopy without histology to confirm endometriosis (Dunselman 2014), the clinical significance of histological verification remains debatable. Diagnosis by surgical visualisation only remains a common clinical practice and can be considered reliable when an accurate 
inspection of the abdominal cavity is performed by experienced surgeons. We chose to include the studies that only reported surgical visualisation as the reference standard and we did not wish to loose potentially valuable information by excluding studies that did not confirm the diagnosis histologically; however this could impact the accuracy of assignation to the case and control groups. Only one study did not report using histology as a part of reference standard and although this could bias the reported results, the impact of including this study on the review findings is likely to be low (Wang 2014).

There are no well-established criteria for replacement or triage diagnostic tests, therefore we chose the criteria that were both realistic and clinically applicable to assist in the interpretation of the complex results. For a replacement test, we considered the threshold reported by the only systematic review on accuracy of the reference standard (laparoscopy) in detecting endometriosis to be the most objective (Wykes 2004). The meta-analysis was published in 2004 and included four eligible studies comprising 433 women. We acknowledge the limitations associated with emphasising a single review, particularly if it does not present the latest and possibly more accurate data that reflect advances in surgical expertise and technology. Several studies on accuracy of laparoscopy in detecting endometriosis have been published in the last decade; however their results were not addressed in a systematic way. A further systematic analysis to determine the accuracy of laparoscopy was beyond the scope of this review. The criteria for triage tests utilised the common concepts of SnOUT and SpIN in medical statistics and the cut-offs were set at levels we considered to be clinically relevant (see Role of index test(s)). We encourage the readers to apply independent interpretations of the presented diagnostic estimates with using thresholds that may be more applicable to specific populations and clinical circumstances.

\section{Applicability of findings to the review question}

QUADAS-2 assigned a low rank to clinical applicability with respect to participant selection in $63 \%$ of the studies (5/8), summarised as a high concern in all these reports. This occurred when the set of participants in the study was broader that seen in clinical practice or when the spectrum of the target condition was limited and the findings may not be applicable to the review question and to clinical practice. Applicability of the index test and reference standard was judged to be satisfactory using the QUADAS-2 tool for all studies. However, the majority of included studies were conducted in academic institutions with a high level of expertise in laboratory techniques and the index test outcome measures may not be able to be reproduced in all institutions or extrapolated to general practice.

Some potentially relevant well-designed studies were excluded as they did not directly address the review question. For example we did exclude studies that reported on biomarkers with differential expression in endometriosis, but that did not provide enough information to assess the diagnostic performance of the biomarker. Some forms of endometriosis, such as bladder, ureteric or involving the extra-pelvic sites (e.g. umbilicus, hernia sacs, abdominal wall, lung, kidney, etc.) were also excluded from the review as they are informed predominantly by case reports or small case series and diagnostic laparoscopy is not an applicable reference test for these conditions. Although these target conditions are rare, from a clinical perspective the diagnostic options for these forms of endometriosis remain unclear.

\section{AUTHORS' CONCLUSIONS}

\section{Implications for practice}

There is not enough evidence to recommend any urinary biomarker for use in clinical practice for the diagnosis of endometriosis. None of the biomarkers qualified for either replacement or triage test and all were assessed in individual studies.

As there is an absence of well-established criteria for an adequate diagnostic test, the diagnostic criteria for replacement and triage tests were determined by the authors of this review in a way that we believe will aid the interpretation for clinically active readers. However we encourage readers to apply different criteria according to each clinical population and situation.

\section{Implications for research}

Currently, randomised controlled treatment trials require women with and without endometriosis to have had diagnostic surgery for accurate group allocation. For ethical reasons, therapeutic surgery is usually performed at the same time, potentially biasing treatment trial outcomes. Thus our current inability to diagnose and assess the progression of endometriosis in a non-invasive way before surgery is a significant limitation in the advancement of clinical research in endometriosis.

The evaluation of biomarkers in urine is a relatively novel research field and only a few urinary tests have been investigated . Further well-designed diagnostic studies are required to establish the test accuracy and clinical utility of urinary peptides and other urinary tests.

The QUADAS quality assessment of the included studies identified several weakness in study design that can impede an objective evaluation of the findings. We recommend that future authors consider: 1 ) including large cohorts after predefining the sample size via a power calculation (Liu 2005); 2) focusing on a 'singlegate' design that only includes a clinically relevant population (Rutjes 2005); 3) utilising a diagnostic accuracy study design that adheres to the recommendations of the Standards for Reporting of Diagnostic Accuracy (STARD) initiative (Bossuyt 2003); 4) incorporating the QUADAS checklist into the study design (Whiting 2011); 5) formally assessing inter-and intra-observer variability of the laboratory methods; 6 ) establishing universally acceptable laboratory methodologies (Rahimoglu 2014), and diagnostic criteria for a positive test; 7 ) utilising universally acceptable methods of performing laparoscopy as the reference standard test (Becker 2014); 8) implementing validation techniques to assess how the results of a statistical analysis will generalise to an independent data set; 9) undertaking direct comparisons of promising tests in conjunction with a cost effectiveness analysis; 10) applying testing to different clinical phenotypes rather than to women classified according to rASRM staging (Vitonis 2014); and 11) assessing the long-term outcomes and lifetime healthcare costs of women that have participated in diagnostic test accuracy trials of specific diagnostic tests.

\section{ACK N O WLEDGEMENTS}

We would like to thank Associate Professor Petra Macaskill for her valuable comments and substantial contribution to development of the statistical methods for the review. Sincere thanks to Professor Ali Akoum for intellectual input and help with drafting of the 
protocol. We are grateful to Marian Showell, the Trials Search Coordinator of the Cochrane Gynaecology and Fertility Group, for her help in designing and conducting the literature search and in locating the full texts of the relevant studies. We gratefully acknowledge the help of the authors of the review series (Deepika
Arora, Devashana Gupta, Lucy Prentice and Rabia Shaikh) for their dedicated assistance in the studies' selection process. Finally, we thank all contacted authors who contributed information to this review. 


\section{R E F E R E N C E S}

\section{References to studies included in this review}

Cho 2007 \{published data only\}

Cho SH, Oh YJ, Nam A, Kim HY, Park JH, Kim JH, et al. Evaluation of serum and urinary angiogenic factors in patients with endometriosis. American Journal of Reproductive Immunology 2007; Vol. 58, issue 6:497-504. [DOI: 10.1111/ j.1600-0897.2007.00535.x; ISSN 1046-7408]

\section{Cho 2012 \{published data only\}}

Cho S, Choi YS, Yim SY, Yang HI, Jeon YE, Lee KE, et al. Urinary vitamin D-binding protein is elevated in patients with endometriosis. Human Reproduction 2012;27(2):515-22.

\section{El-Kasti 2011 \{published data only\}}

* El-Kasti MM, Wright C, Fye HK, Roseman F, Kessler BM, Becker CM. Urinary peptide profiling identifies a panel of putative biomarkers for diagnosing and staging endometriosis. Fertility and Sterility 2011; Vol. 95, issue 4:1261-6. [DOI: 10.1016/ j.fertnstert.2010.11.066; ISSN 1556-5653]

\section{Kuessel 2014 \{published data only\}}

Kuessel L, Jaeger-Lansky A, Pateisky P, Rossberg N, Schulz A, Schmitz AA, et al. Cytokeratin-19 as a biomarker in urine and in serum for the diagnosis of endometriosis--a prospective study. Gynaecological Endocrinology 2014; Vol. 30, issue 1:38-41. [DOI: 10.3109/09513590.2013.856409; ISSN 1473-0766]

\section{Lessey 2014 \{published data only\}}

* Lessey BA, Savaris RF, Ali S, Brophy S, Tomazic-Allen S, Chwalisz K. Diagnostic Accuracy of Urinary Cytokeratin 19 Fragment for Endometriosis. Reproductive Sciences 2014 Oct 08 [Epub ahead of print]. [DOI: 10.1177/1933719114553064; ISSN 1933-7205 (Electronic)]

\section{Potlog-Naharia 2004 \{published data only\}}

Potlog-Nahari C, Stratton P, Winkel C, Widra z, Sinaii N, Connors S, et al. Urine vascular endothelial growth factor$A$ is not a useful marker for endometriosis. Fertility and Sterility 2004; Vol. 81, issue 6:1507-12. [DOI: 10.1016/ j.fertnstert.2003.10.040; ISSN 0015-0282]

\section{Wang 2014 \{published data only\}}

Wang L, Liu HY, Shi HH, Lang JH, Sun W. Urine peptide patterns for non-invasive diagnosis of endometriosis:a preliminary prospective study. European Journal of Obstetrics \& Gynecology and Reproductive Biology 2014;177:23-8. [DOI: 10.1016/ j.ejogrb.2014.03.011; ISSN 1872-7654 (Electronic)]

Yun 2014 \{published data only\}

* Yun BH, Lee YS, Chon SJ, Jung YS, Yim SY, Kim HY, et al. Evaluation of elevated urinary enolase I levels in patients with endometriosis. Biomarkers 2014; Vol. 19, issue 1:16-21. [DOI: 10.3109/1354750X.2013.863973; ISSN 1366-5804]

\section{References to studies excluded from this review}

Gjavotchanoff 2015 \{published data only\}

Gjavotchanoff R. CYFRA 21-1 in urine: a diagnostic marker for endometriosis?. International Journal of Women's Health 2015;7:205.

\section{Itoh 2007 \{published data only\}}

Itoh H, Iwasaki M, Hanaoka T, Sasaki H, Tanaka T, Tsugane S. Urinary bisphenol-A concentration in infertile Japanese women and its association with endometriosis: A crosssectional study. Environmental Health and Preventive Medicine 2007;12(6):258-64.

\section{Itoh 2008 \{published data only\}}

Itoh H, Iwasaki M, Nakajima Y, Endo Y, Hanaoka T, Sasaki H, et al. A case-control study of the association between urinary cadmium concentration and endometriosis in infertile Japanese women. Science of the Total Environment 2008:402(2-3):171-5

Itoh 2009 \{published data only\}

Itoh H, Iwasaki M, Hanaoka T, Sasaki H, Tanaka T, Tsugane S. Urinary phthalate monoesters and endometriosis in infertile Japanese women. Science of the Total Environment 2009;408(1):37-42.

\section{Sharma 2010 \{published data only\}}

Sharma I, Dhaliwal LK, Saha SC, Sangwan S, Dhawan V. Role of 8-iso-prostaglandin F2alpha and 25-hydroxycholesterol in the pathophysiology of endometriosis. Fertility \& Sterility 2010; Vol. 94, issue 1:63-70. [DOI: 10.1016/j.fertnstert.2009.01.141; ISSN 1556-5653]

\section{Williams 2014 \{published data only\}}

Williams KE, Miroshnychenko O, Johansen EB, Niles RK, Sundaram R, Kannan K, et al. Urine, peritoneal fluid and omental fat proteomes of reproductive age women: Endometriosis-related changes and associations with endocrine disrupting chemicals. Journal of Proteomics 2015;113:194-201. [DOI: 10.1016/j.jprot.2014.09.015; ISSN 1876-7737 (Electronic)]

\section{References to studies awaiting assessment}

Becker 2010 \{published data only\}

Becker CM, Louis G, Exarhopoulos A, Mechsner S, Ebert AD, Zurakowski D, et al. Matrix metalloproteinases are elevated in the urine of patients with endometriosis. Fertility and Sterility 2010;94(6):2343-6.

Tokushige 2011 \{published data only\}

Tokushige N, Markham R, Crossett B, Ahn SB, Nelaturi VL, Khan $A$, et al. Discovery of a novel biomarker in the urine in women with endometriosis. Fertility and Sterility 2011; Vol. 95, issue 1:46-9. [DOI: 10.1016/j.fertnstert.2010.05.016; ISSN 1556-5653] 


\section{References to ongoing studies}

NCT02337816 \{published data only (unpublished sought but not used)\}

Role of Metabolomics in the Diagnosis of

EndometriosisClinicalTrials.gov Identifier: NCT023378160ther study name: ENDOMETAB01. Ongoing study December 2014.

\section{Additional references}

\section{ACOG 2010}

The American College of Obstetricians and Gynecologists. Practice Bulletin No. 114: Management of Endometriosis.. Obstetrics and Gynecology 2010;116(1):223-36.

\section{Adamson 2008}

Adamson GD. Endometriosis Fertility Index (EFI): the new validated endometriosis staging system. WCE 2008, Art \& Science of Endometriosis. Melbourne, Australia, 11-14 March, 2008. Poster \# 33, Abstract page 7.2.

\section{Almeida Filho 2008}

Almeida Filho DP, Oliveira LJ, Amaral VF. Accuracy of laparoscopy for assessing patients with endometriosis. Sao Paulo Medical Journal 2008;126(6):305-8.

\section{American Society for Reproductive Medicine 1997}

American Society for Reproductive Medicine. Revised American Society for Reproductive Medicine classification of endometriosis: 1996. Fertility and Sterility 1997;67(5):817-21.

\section{Ballard 2008}

Ballard KD, Seaman HE, de Vries CS, Wright JT. Can symptomatology help in the diagnosis of endometriosis? Findings from a national case-control study - Part 1. BJOG 2008;115(11):1382-91.

\section{Batt 2003}

Batt R, Mitwally MF. Endometriosis from thelarche to midteens: pathogenesis and prognosis, prevention and pedagogy. Journal of Pediatric and Adolescent Gynecology 2003;16(6):337-47.

\section{Becker 2014}

Becker CM, Laufer MR, Stratton P, Hummelshoj I, Missmer SA, Zondervan KT, et al. World Endometriosis Research Foundation Endometriosis Phenome and biobanking harmonization project: I. Surgical phenotype data collection in endometriosis research. Fertility and Sterility 2014;102(5):1213-22.

\section{Bossuyt 2003}

Bossuyt PM, Reitsma JB, Bruns DE, Gatsonis CA, Glasziou PP, Irwig LM, et al. Standards for Reporting of Diagnostic Accuracy. Towards complete and accurate reporting of studies of diagnostic accuracy: the STARD initiative. BMJ 2003;326(7379):41-4.

\section{Bossuyt 2008}

Bossuyt PM, Leeflang MM. Chapter 6: Developing Criteria for Including Studies. Cochrane Handbook for Systematic Reviews of Diagnostic Test Accuracy Version 0.4 [updated September 2008]. The Cochrane Collaboration, 2008.

\section{Chapron 2003a}

Chapron C, Fauconnier A, Vieira M, Barakat Dousset HB, Pansini V, Vacher-Lavenu MC, et al. Anatomical distribution of deeply infiltrating endometriosis: surgical implications and proposition for a classification. Human Reproduction 2003;18(1):157-61.

\section{Chapron 2003b}

Chapron C, Fauconnier A, Dubuisson JB, Barakat H, Vieira M, Bréart G. Deep infiltrating endometriosis: relation between severity of dysmenorrhea and extent of disease. Human Reproduction 2003;18:760-6.

\section{Chapron 2003c}

Chapron C, Cravello L, Chopin N, Kreiker G, Blanc B, Dubuisson JB. Complications during set-up procedures for laparoscopy in gynecology: open laparoscopy does not reduce the risk of major complications. Acta Obstetrica et Gynecologica Scandinavica 2003;82:1125-9.

\section{D'Hooghe 2001}

D'Hooghe TM, Bambra CS, Xiao L, Peixe K, Hill JA. Effect of menstruation and intrapelvic injection of endometrium on inflammatory parameters of peritoneal fluid in the baboon (Papio anubis and Papio cynocephalus). American Journal of Obstetrics \& Gynecology 2001;184(5):917-25.

\section{de Vet 2008}

de Vet HCW, Eisinga A, Riphagen II, Aertgeerts B, Pewsner D. Chapter 7: Searching for Studies. Cochrane Handbook for Systematic Reviews of Diagnostic Test Accuracy Version 0.4 [updated September 2008]. The Cochrane Collaboration, 2008.

\section{Dmowski 1997}

Dmowski WP, Lesniewicz R, Rana N, Pepping P, Noursalehi M. Changing trends in the diagnosis of endometriosis: a comparative study of women with pelvic endometriosis presenting with chronic pelvic pain or infertility. Fertility and Sterility 1997;67(2):238-43.

\section{Dunselman 2014}

Dunselman GA, Vermeulen N, Becker C, Calhaz-Jorge C, D'Hooghe T, De Bie B, et al. ESHRE guideline: management of women with endometriosis. Human Reproduction 2014;29(3):400-12.

\section{Eskenazi 2001}

Eskenazi B, Warner M, Bosignore L, Olive D, Samuels S, Vercellini P. Validation study of nonsurgical diagnosis of endometriosis. Fertility and Sterility 2001;76(5):929-35.

\section{Fassbender 2015}

Fassbender A, Burney RO, Dorien FO, D'Hooghe T, Giudice L. Update on Biomarkers for the Detection of Endometriosis. BioMed Research International (http://www.hindawi.com/ journals/bmri/2015/130854/) (accessed 20 April 2015).

\section{Fauconnier 2005}

Fauconnier A, Chapron C. Endometriosis and pelvic pain: epidemiological evidence of the relationship and implications. Human Reproduction Update 2005;11(6):595-606. 


\section{Frishman 2006}

Frishman GN, Salak JR. Conservative surgical management of endometriosis in women with pelvic pain. Journal of Minimally Invasive Gynecology 2006;13(6):546-58.

\section{Gao 2006}

Gao X, Yeh YC, Outley J, Simon J, Botteman M, Spalding J. Health-related quality of life burden of women with endometriosis: a literature review. Current Medical Research \& Opinion 2006;22(9):1787-97.

\section{Giudice 2004}

Giudice LC, Kao LC. Endometriosis. Lancet 2004;364(9447):1789-99.

\section{Guo 2009}

Guo SW. Recurrence of endometriosis and its control. Human Reproduction Update 2009;15(4):441-61.

\section{Guzick 1997}

Guzick DS, Silliman NP, Adamson GD, Buttram VC Jr, Canis M, Malinak LR, et al. Prediction of pregnancy in infertile women based on the American Society for Reproductive Medicines revised classification of endometriosis. Fertility and Sterility 1997;67(5):822-9.

\section{Halme 1984}

Halme J, Hammond MG, Hulka JF, Raj SG, Talbert LM. Retrograde menstruation in healthy women and in patients with endometriosis. Obstetrics \& Gynecology 1984;64(2):151-4.

\section{Hull 2008}

Hull ML, Escareno CR, Godsland JM, Doig JR, Johnson CM, Phillips SC, et al. Endometrial-peritoneal interactions during endometriotic lesion establishment. American Journal of Pathology 2008;173(3):700-15.

\section{Johnson and Hummelshoj 2013}

Johnson NP, Hummelshoj L, World Endometriosis Society Montpellier Consortium. Consensus on current management of endometriosis. Human Reproduction 2013;28(6):1252-68.

\section{Kao 2003}

Kao LC, Germeyer A, Tulac S, Lobo S, Yang JP, Taylor RN, et al. Expression profiling of endometrium from women with endometriosis reveals candidate genes for diseasebased implantation failure and infertility. Endocrinology 2003;144(7):2870-81.

\section{Kennedy 2005}

Kennedy S, Bergqvist A, Chapron C, D'Hooghe T, Dunselman G, ESHRE Special Interest Group for Endometriosis. ESHRE guideline for the diagnosis and treatment of endometriosis. Human Reproduction 2005;20(10):2698-704.

\section{Koninckx 1991}

Koninckx PR, Meuleman C, Demeyere S, Lesaffre E, Cornillie FJ. Suggestive evidence that pelvic endometriosis is a progressive disease, whereas deeply infiltrating endometriosis is associated with pelvic pain. Fertility and Sterility 1991;55(4):759-65

\section{Ling 1999}

Ling F. Randomized controlled trial of depot leuprolide in patients with chronic pelvic pain and clinically suspected endometriosis. Obstetrics \& Gynecology 1999;93(1):51-8.

\section{Liu 2005}

Liu A, Schisterman EF, Mazumdar M, Hu J. Power and sample size calculation of comparative diagnostic accuracy studies with multiple correlated test results. Biometrical Journal 2005;47(2):140-50.

\section{Marchino 2005}

Marchino GL, Gennarelli G, Enria R, Bongioanni F, Lipari G, Massobrio M. Diagnosis of pelvic endometriosis with use of macroscopic versus histologic findings. Fertility and Sterility 2005;84(1):12-5.

\section{Martin 2006}

Martin DC. Applying STARD criteria to the laparoscopic identification of endometriosis (abstract). Fertility and Sterility. 2006; Vol. 86 Suppl 2:270.

\section{Matsuzaki 2006}

Matsuzaki S, Canis M, Pouly JL, Rabischong B, Botchorishvili R, Mage $G$. Relationship between delay of surgical diagnosis and severity of disease in patients with symptomatic deep infiltrating endometriosis. Fertility and Sterility 2006;86(5):1314-6.

\section{May 2010}

May KE, Conduit-Hulbert SA, Villar J, Kirtley S, Kennedy SH, Becker CM. Peripheral biomarkers of endometriosis: A systematic review. Human Reproduction Update 2010;16(6):651-74

\section{McGraw-Hill Dictionary of Medicine 2006}

Segen JC (author). McGraw-Hill Concise Dictionary of Modern Medicine. The McGraw-Hill Companies, Inc, 2006.

\section{Medeiros 2009}

Medeiros LR, Rosa DD, Bozzetti MC, Fachel JM, Furness S, Garry R, et al. Laparoscopy versus laparotomy for benign ovarian tumour. Cochrane Database of Systematic Reviews 2009, Issue 2. [DOI: 10.1002/14651858.CD004751.pub3; ISSN 1469-493X (electronic)]

\section{Nyholt 2012}

Nyholt DR, Low SK, Anderson CA, Painter JN, Uno S, Morris AP, et al. Genome-wide association meta-analysis identifies new endometriosis risk loci. Nature Genetics 2012;44(12):1355-9.

\section{Rahimoglu 2014}

Rahmioglu N, Fassbender A, Vitonis AF, Tworoger SS, Hummelshoj L, D'Hooghe TM, et al. World Endometriosis Research Foundation Endometriosis Phenome and biobanking harmonization project: III. Fluid biospecimen collection, processing, and storage in endometriosis research. Fertility and Sterility 2014;102(5):1233-43. 


\section{Redwine 2003}

Redwine DB. 'Invisible' microscopic endometriosis: a review. Gynecologic and Obstetric Investigation 2003;55(2):63-7.

\section{Rogers 2009}

Rogers PA, D'Hooghe TM, Fazleabas A, Gargett CE, Giudice LC, Montgomery GW, et al. Priorities for Endometriosis Research: recommendations from an international consensus workshop. Reproductive Sciences 2009;16(4):335-46.

\section{Rutjes 2005}

Rutjes AWS, Reitsma JB, Vandenbroucke JP, Glas AS, Bossuyt PMM. Case-control and two-gate designs in diagnostic accuracy studies. Clinical Chemistry 2005;51(8):1335-41.

\section{Sampson 1927}

Sampson JA. Peritoneal endometriosis due to menstrual dissemination of endometrial tissue into the peritoneal cavity. American Journal of Obstetrics \& Gynecology 1927;14(4):422-69.

\section{Simoens 2012}

Simoens S, Dunselman G, Dirksen C, Hummelshoj L, Bokor A, Brandes I, et al. The burden of endometriosis: costs and quality of life of women with endometriosis and treated in referral centres. Human Reproduction 2012;27(5):1292-9.

\section{Sinaii 2002}

Sinaii N, Cleary SD, Ballweg M, Nieman LK, Stratton P. High rates of autoimmune and endocrine disorders, fibromyalgia, chronic fatigue syndrome and atopic diseases among women with endometriosis: a survey analysis. Human Reproduction 2002;17(10):2715-24.

\section{SOGC 2010}

Society of Obstetricians Gynaecologists of Canada. Endometriosis: diagnosis and management. SOGC clinical practice guideline no. 244. Journal of Obstetrics and Gynaecology Canada 2010;32:S1-S28.

\section{Somigliana 2006}

Somigliana E, Vigano P, Parazzini F, Stoppelli S, Giambattista E, Vercellini P. Association between endometriosis and cancer: a comprehensive review and a critical analysis of clinical and epidemiological evidence. Gynecologic Oncology 2006;101(2):331-41.

\section{Spaczynski 2003}

Spaczynski RZ, Duleba AJ. Diagnosis of endometriosis. Seminars in Reproductive Medicine 2003;21(2):193-208.

\section{Stegmann 2008}

Stegmann BJ, Sinaii N, Liu S, Segars J, Merino M, Nieman LK, et al. Using location, color, size, and depth to characterize

\section{CHARACTERISTICS OF STUDIES}

Characteristics of included studies [ordered by study ID] and identify endometriosis lesions in a cohort of 133 women. Fertility and Sterility 2008;89(6):1632-6.

\section{The Gale Encyclopedia of Medicine 2008}

Donna Olendorf (Editor), Christine Jeryan (Editor), Karen Boyden (Editor), Gale Group (Corporate Author). The Gale Encyclopedia of Medicine (5 volume set). The Gale Group, Inc, 2008.

\section{Vercellini 1996}

Vercellini P, Trespidi L, De Giorgi O, Cortesi I, Parazzini F, Crosignani GP. Endometriosis and pelvic pain: relation to disease stage and localization. Fertility and Sterility 1996;65(2):299-304.

\section{Vigano 2004}

Vigano P, Parazzini F, Somigliana E, Vercellini P. Endometriosis: epidemiology and aetiological factors. Best Practice \& Research: Clinical Obstetrics \& Gynaecology 2004;18(2):177-200.

\section{Vitonis 2014}

Vitonis AF, Vincent K, Rahmioglu N, Fassbender A, Buck Louis G, Hummelshoj L, et al. World Endometriosis Research Foundation Endometriosis Phenome and biobanking harmonization project: II. Clinical and covariate phenotype data collection in endometriosis research. Fertility and Sterility 2014;102(5):1223-32.

\section{Whiting 2005}

Whiting PF, Harbord R, Kleijnen J. No role for quality scores in systematic reviews of diagnostic accuracy studies. BMC Medical Research Methodology 2005;5:19.

\section{Whiting 2011}

Whiting PF, Rutjes AW, Westwood ME, Mallett S, Deeks JJ, Reitsma JB, et al. QUADAS-2: A Revised Tool for the Quality Assessment of Diagnostic Accuracy Studies. Annals of Internal Medicine 2011;155(8):529-36.

\section{Wykes 2004}

Wykes CB, Clark TJ, Khan KS. Accuracy of laparoscopy in the diagnosis of endometriosis: a systematic quantitative review. BJOG 2004;111(11):1204-12.

\section{Yeung 2009}

Yeung PP Jr, Shwayder J, Pasic RP. Laparoscopic management of endometriosis: comprehensive review of best evidence. Journal of Minimally Invasive Gynecology 2009;16(3):269-81.

* Indicates the major publication for the study 
Cho 2007

\section{Study characteristics}

Patient sampling Primary objective: To evaluate serum and urinary levels of VEGF, TNF-a and sFlt-1 in patients with endometriosis

Study population: Women who underwent laparoscopy or laparotomy for different indications including pelvic masses, pelvic pain, suspicious endometriosis, infertility and diagnostic evaluation

Selection criteria: Inclusion criteria: pre-menopausal age

Study design: Cross-sectional, two-gate design, prospective collection of samples

Patient characteristics and setting
Clinical presentation: Pelvic pain, infertility, pelvic mass, other not specified

Age: Mean age $32.65 \pm 6.82$ years (endometriosis group), $30.96 \pm 6.36$ years (controls)

Number of participants enrolled: 70 women

Number of participants available for analysis: 70 women (in follicular or luteal cycle phase, numbers not specified)

Setting: Department of O\&G, Yongdong Severance Hospital, Yonsei University College of Medicine

Place of study: Seoul, Korea

Period of study: Not stated

Language: English

Index tests

Index test: Urinary vascular endothelial growth factor (VEGF), tumour necrosis factor-alpha (TNF-a) and soluble fms like tyrosine kinase (sFlt-1)

Details of the index test procedure as stated: Urine concentrations of VEGF, sFlt-1, and TNF-a, were measured using specific commercial sandwich ELISA kit according to manufacturer protocols (Quantikine; R\&D systems Inc, MN, USA); sample processing described

Threshold for positive result: Not provided

Examiners: No information provided; unclear if blinded to the result of reference standard

Interobserver variability: Not reported

Target condition and reference standard(s)

\section{Target condition: Endometriosis}

Prevalence of target condition in the sample: $n=46 / 70(66 \%)$ : stage I-II 15, stage III-IV 31; controls $n$ $=24$

Reference standard: Laparoscopy/Laparotomy and histology

Description of positive case definition by reference standard as reported: Visual inspection, confirmed by histopathology; staging according to the rASRM classification

Examiners: No information provided
Time interval between index test and reference standard: Urine sample was collected after induction of anaesthesia

Withdrawals: None

\section{Comparative}




\section{Cho 2007 (Continued)}

Key conclusions by the authors

The pathogenesis of minimal-to-mild endometriosis and moderate-to severe endometriosis seems to be different. Increased sFlt-1 levels in serum and urine of minimal-to-mild disease indicate that sFlt-1 may have an important role in inhibiting angiogenic process of the disease

\section{Methodological quality}

\begin{tabular}{llll}
\hline Item & Authors' judgement & Risk of bias & Applicability concerns \\
\hline
\end{tabular}

DOMAIN 1: Patient Selection

Was a consecutive or ran- $\quad$ Unclear
dom sample of patients en-
rolled?

Did the study avoid inap- Yes
propriate exclusions?

Was a 'two-gate' design No
avoided?

For VEGF and TNF-a there was no statistically significant difference between the groups - no data available for meta-analysis

Urinary levels for all biomarkers were corrected for creatinine

Urinary VEGF and TNF-a levels were unaffected by severity of endometriosis or menstrual cycle phase

For sFlt-1 there was statistically significant difference between the groups, but there was insufficient data to construct $2 \times 2$ tables - not included in this review

\begin{tabular}{lll}
\hline & High & High \\
\hline
\end{tabular}

\section{DOMAIN 2: Index Test All tests}

\section{Were the index test results Unclear}

interpreted without knowl-

edge of the results of the

reference standard?

\footnotetext{
If a threshold was used, was No

it pre-specified?
}

Was a cycle phase consid- Yes

ered in interpretation of the

result of index test

High Low

\section{DOMAIN 3: Reference Standard}

Is the reference standards Yes

likely to correctly classify

the target condition?

Were the reference stan- Yes

dard results interpreted 
Cho 2007 (Continued) without knowledge of the results of the index tests?

\begin{tabular}{lll}
\hline & Low \\
\hline DOMAIN 4: Flow and Timing & \\
\hline $\begin{array}{l}\text { Was there an appropriate } \\
\text { interval between index test } \\
\text { and reference standard? }\end{array}$ \\
\hline $\begin{array}{l}\text { Did all patients receive the } \\
\text { same reference standard? }\end{array}$ \\
\hline $\begin{array}{l}\text { Were all patients included } \quad \text { Yes } \\
\text { in the analysis? }\end{array}$ \\
\hline
\end{tabular}

\section{Cho 2012}

\section{Study characteristics}

Patient sampling

Primary objective: To investigate proteins secreted in urine of patients with endometriosis using proteomic techniques in order to identify potential markers for the clinical diagnosis of endometriosis; to evaluate urinary VDBP in patients with endometriosis

Study population: Women who underwent laparoscopy for various indications including pelvic masses, pelvic pain, suspicious endometriosis, infertility and diagnostic evaluation

Selection criteria: Inclusion criteria: pre-menopausal age; exclusion criteria: previous hormone or GnRH agonist use, adenomyosis, endometrial cancer, hyperplasia or endometrial polyps, infectious diseases, chronic or acute inflammatory diseases, malignancy, autoimmune disease and cardiovascular disease

Study design: Cross-sectional, single-gate design, prospective collection of samples

Patient characteristics Clinical presentation: Pelvic masses, pelvic pain, suspicious endometriosis, infertility and setting

Age: Mean age $34.22 \pm 6.88$ years (endometriosis group), $32.76 \pm 10.26$ years (control group)

Number of participants enrolled: 95 women

Number of participants available for analysis: 95 women (in follicular or luteal cycle phase, numbers not specified)

Setting: Gangnam Severance Hospital

Place of study: Seoul, Korea

Period of study: January 2008 to October 2010

Language: English

Index tests

Index test: Urinary vitamin D binding protein (VDBP-Cr)

Details of the index test procedure as stated: The urinary concentration of VDBP was measured using specific commercial sandwich ELISA assays according to manufacturer's protocols (ALPCO Diagnostics, Salem, $\mathrm{NH}$, USA); urine VDBP values were normalized to urine $\mathrm{Cr}$ concentrations

Threshold for positive result: Cut-off value $>87.83 \mathrm{ng} / \mathrm{mgCr}$ using AUC of 0.678 , not pre-specified 
Cho 2012 (Continued)

Examiners: No information provided; unclear if blinded to the result of reference standard

Interobserver variability: Not reported

Target condition and reference standard(s)

\section{Target condition: Endometriosis}

Prevalence of target condition in the sample: $n=57 / 95$ (60\%): stage I-II 5, stage III-IV 52; controls $n=38$

Reference standard: Laparoscopy and histology

Description of positive case definition by reference standard as reported: Visual inspection, confirmed by histopathology; staging according to the rASRM classification

Examiners: No information provided

Flow and timing

Time interval between index test and reference standard: Urine sample was collected when the bladder was catheterized after induction of anaesthesia

Withdrawals: None reported

\begin{tabular}{ll}
$\begin{array}{l}\text { Key conclusions by the } \\
\text { authors }\end{array}$ & $\begin{array}{l}\text { Urinary VDBP levels are elevated in patients with endometriosis, but they have limited value as a potential } \\
\text { diagnostic biomarker for endometriosis (sensitivity 58\%, specificity 76\%) }\end{array}$ \\
\hline Conflict of interest & $\begin{array}{l}\text { The authors reported no conflict of interests; supported by the Basic Science Research Program of NRF of } \\
\text { Korea by the Ministry of Education, Science and Technology (2010-0023323) }\end{array}$
\end{tabular}

Notes

The reported diagnostic estimates for urinary VDBP levels were corrected for creatinine (VDBP-Cr)

VDBP-Cr was significantly higher in patients with endometriosis during the secretory phase, but no different in proliferative phase

VDBP-Cr did not correlate with severity of endometriosis

Combined estimates for VDBP-Cr + serum Ca-125 were also reported but not presented in this review

Using the urinary proteomic analysis, enolase-I, a-1 antitrypsin, prealbumin and Bb' domain of disulfide isomerase were also found to be increased in urine of patients with endometriosis, but were not evaluated in this study.

\section{Methodological quality}

\begin{tabular}{llll}
\hline Item & Authors' judgement & Risk of bias & Applicability concerns
\end{tabular}

\section{DOMAIN 1: Patient Selection}

Was a consecutive or Unclear
random sample of pa-

tients enrolled?

\section{Did the study avoid in- Yes}

appropriate exclusions?

Was a 'two-gate' design Yes avoided?

\section{DOMAIN 2: Index Test All tests}


Cho 2012 (Continued)

Were the index test results interpreted without knowledge of the results of the reference standard?

If a threshold was used, No was it pre-specified?

Was a cycle phase conYes sidered in interpretation of the result of index test

\section{DOMAIN 3: Reference Standard}

Is the reference stan- Yes

dards likely to correctly

classify the target con-

dition?

\section{Were the reference} Yes standard results interpreted without knowledge of the results of the index tests?

\begin{tabular}{|c|c|c|}
\hline & Low & Low \\
\hline \multicolumn{3}{|c|}{ DOMAIN 4: Flow and Timing } \\
\hline $\begin{array}{l}\text { Was there an appropri- } \\
\text { ate interval between in- } \\
\text { dex test and reference } \\
\text { standard? }\end{array}$ & Yes & \\
\hline $\begin{array}{l}\text { Did all patients receive } \\
\text { the same reference } \\
\text { standard? }\end{array}$ & Yes & \\
\hline \multirow[t]{2}{*}{$\begin{array}{l}\text { Were all patients in- } \\
\text { cluded in the analysis? }\end{array}$} & Yes & \\
\hline & Low & \\
\hline
\end{tabular}

\section{El-Kasti 2011}

\section{Study characteristics}

Patient sampling

Primary objective: To identify a potential diagnostic endometriosis marker using matrix-enhanced laser desorption/ionization time-of-flight mass spectrometry (MALDI-TOF-MS)-based urinary proteomics

Study population: Women undergoing laparoscopy for investigation of abdominal/pelvic pain or subfertility 
El-Kasti 2011 (Continued)

Selection criteria: Not specified

Study design: Cross-sectional, single-gate design, prospective collection of samples

Patient characteristics Clinical presentation: Presenting with abdominal/pelvic pain or subfertility

and setting

Age: Mean age 35 years, range 20 to 50 years

Number of participants enrolled: 53

Number of participants available for analysis: 39 (in follicular, peri-ovulatory or/and luteal phase); only moderate/severe endometriosis included in analysis)

Setting: University hospital, tertiary referral centre for endometriosis, Department of O\&G, University of Oxford

Place of study: Oxford, United Kingdom

Period of study: Not stated

Language: English

Index tests

Index test: Urinary proteome (peptide m/z 1767.1 Da and peptide m/z 1824.3 Da)

Description of positive case definition by index test as reported: Spectra were acquired on a MALDITOF UltraFlex mass spectrometer in linear mode at $80 \%$ laser power over a range of 720 to 10,000 Daltons (Da) for a total of 1200 laser shots; spectra were analysed using FlexAnalysis 2.4 (Bruker Daltonics); groupspecific peaks were identified by support vector machine (SVM) algorithm method; computational models were generated that function as classifiers

Threshold for positive result: For peptide m/z 1767.1 Da $\geq 35.22$ au; for peptide m/z 1824.3 Da $\geq 29.34$ au, not pre-specified

Examiners: No information provided; the examiners were blinded to the clinical information

Interobserver variability: Not stated

Target condition and reference standard(s)
Target condition: Endometriosis

Prevalence of target condition in the sample: $n=23 / 53(43 \%)$ : stage I-IV: stage I-II 23 samples, stage IIIIV 37 samples; controls $n=30$ (44 samples); participants donated several samples across the cycle

Reference standard: Laparoscopy and histology

Description of positive case definition by reference test as reported: Visual inspection confirmed by histology; staging by rASRM scoring system

Examiners: Experienced surgeons blinded to index test result

Flow and timing

Time interval between index test and reference standard: Time frame between index test and reference unclear, but from the context it looks like it was close to surgery time

Withdrawals: 14 control patients were excluded for analysis as had other pelvic pathology

Comparative

Key conclusions by the Urinary proteomic analysis may provide a novel method of diagnosing and staging endometriosis authors
Conflict of interest
The authors declared no conflict of interests; supported by MRC New Investigator Award (G0601458) and funding from the Department of Health's NIHR Biomedical Research Centres Scheme 
El-Kasti 2011 (Continued)

Notes
The reported diagnostic estimates for both urinary peptides were only for moderate-severe endometriosis and for specific cycle phases

The diagnostic estimates for differentiation of minimal-mild from moderate-severe disease are not presented in this review

During the follicular phase or when comparing controls with minimal/mild endometriosis patients, no statistically significant difference was detected regarding the peptide profiles

The authors identified 4 additional peptides that significantly differed in moderate-severe endometriosis (m/z 1519.3,

2660.8, and 9767.6 Da in peri-ovulatory phase and 3265.4 Da in luteal phase), but did not assess the diagnostic performance of these markers

\title{
Methodological quality
}

\begin{tabular}{llll}
\hline Item & Authors' judgement & Risk of bias & Applicability concerns \\
\hline
\end{tabular}

DOMAIN 1: Patient Selection

Was a consecutive or Unclear
random sample of pa-

tients enrolled?

Did the study avoid in- $\quad$ Unclear
appropriate exclusions?

Was a 'two-gate' design Yes avoided?

\section{DOMAIN 2: Index Test All tests}

\section{Were the index test re- Yes}

sults interpreted with-

out knowledge of the

results of the reference

standard?

\section{If a threshold was used, No}

was it pre-specified?

Was a cycle phase con- Yes

sidered in interpreta-

tion of the result of in-

dex test

\begin{tabular}{lll}
\hline & High & Low \\
\hline
\end{tabular}

\section{DOMAIN 3: Reference Standard}

\author{
Is the reference stan- \\ dards likely to correctly \\ classify the target con- \\ dition?
}

\section{Yes}

Were the reference Yes

standard results inter- 
El-Kasti 2011 (Continued) preted without knowledge of the results of the index tests?

Low

Low

\section{DOMAIN 4: Flow and Timing}

Was there an appropri- Unclear ate interval between index test and reference standard?

\section{Did all patients receive Yes}

the same reference

standard?

Were all patients inNo

cluded in the analysis?

\section{High}

\section{Kuessel 2014}

\section{Study characteristics}

Patient sampling

Primary objective: To evaluate the usefulness of urinary CK19 as biomarker for the diagnosis of endometriosis

Study population: Women who underwent laparoscopy for suspected endometriosis, pelvic pain, benign adnexal masses or leiomyoma uteri

Selection criteria: Inclusion criteria: age 18 to 50 years; exclusion criteria: known infectious or chronic autoimmune disease

Study design: Cross-sectional, two-gate design, prospective collection of samples

Patient characteristics and setting
Clinical presentation: Suspected endometriosis, pelvic pain, benign adnexal masses or leiomyoma uteri; 8 participants from study and 4 from control group received hormonal therapy within 3 months before surgery

Age: Mean age $33.9 \pm 7.8$ years (endometriosis group), $36.8 \pm 7.4$ years (controls)

Number of participants enrolled: 76 women

Number of participants available for analysis: 76 women (49 in follicular and 27 in luteal cycle phase)

Setting: Department of O\&G, Medical University of Vienna

Place of study: Vienna, Austria

Period of study: Not stated

Language: English

Index tests 
Details of the index test procedure as stated: Urine concentrations of CK 19 were measured using sandwich ELISA TM-Cyfra21-1 (DRG Instruments GmbH, Marburg, Germany); sample processing described

Threshold for positive result: Not provided

Examiners: No information provided; unclear if blinded to the result of reference standard

Interobserver variability: Not reported

Target condition and reference standard(s)
Target condition: Endometriosis

Prevalence of target condition in the sample: $n=44 / 76(58 \%)$ : severity not reported; controls $n=$ 32

Reference standard: Laparoscopy and histology

Description of positive case definition by reference standard as reported: Visual inspection, confirmed by histopathology

Examiners: No information provided
Time interval between index test and reference standard: Urine sample was collected during surgery

Withdrawals: None

\begin{tabular}{ll}
\hline Comparative & $\begin{array}{l}\text { There was no statistically significant correlation between the concentration of CK 19 in urine and } \\
\text { the diagnosis of endometriosis. Assigning the samples to either proliferative or secretory phase } \\
\text { of the cycle did not change the finding. Further studies are warranted to explore the usefulness of } \\
\text { CK19 in the diagnosis of endometriosis. }\end{array}$ \\
\hline Conflict of interest & $\begin{array}{l}\text { 3 of the authors are employees of pharmaceutical company, the authors declared no conflict of in- } \\
\text { terests; supported by Bayer Pharma AG }\end{array}$ \\
\hline Notes & $\begin{array}{l}\text { For CK } 19 \text { there was no statistically significant difference between the groups - no data available for } \\
\text { meta-analysis }\end{array}$ \\
Urinary CK 19 levels were not corrected for creatinine \\
Urinary CK 19 levels were unaffected by menstrual cycle phase \\
Severity of the disease was not reported
\end{tabular}

\section{Methodological quality}

\begin{tabular}{|c|c|c|c|}
\hline Item & Authors' judgement & Risk of bias & Applicability concerns \\
\hline \multicolumn{4}{|l|}{ DOMAIN 1: Patient Selection } \\
\hline $\begin{array}{l}\text { Was a consecutive or random } \\
\text { sample of patients enrolled? }\end{array}$ & Unclear & & \\
\hline $\begin{array}{l}\text { Did the study avoid inappro- } \\
\text { priate exclusions? }\end{array}$ & Yes & & \\
\hline
\end{tabular}

Was a 'two-gate' design avoid- No ed? 
Kuessel 2014 (Continued)

High

High

\section{DOMAIN 2: Index Test All tests}

Were the index test results in- Unclear terpreted without knowledge of the results of the reference standard?

If a threshold was used, was it No pre-specified?

Was a cycle phase considered Yes in interpretation of the result of index test

\begin{tabular}{lll}
\hline & High & Low \\
\hline
\end{tabular}

\section{DOMAIN 3: Reference Standard}

Is the reference standards like- Yes ly to correctly classify the target condition?

Were the reference standard Yes results interpreted without knowledge of the results of the index tests?

\section{Low}

\section{DOMAIN 4: Flow and Timing}

Was there an appropriate in- Yes
terval between index test and reference standard?

Did all patients receive the $\quad$ Yes
same reference standard?

same reference standard?

Were all patients included in Yes

the analysis?

\section{Low}

\section{Lessey 2014}

\section{Study characteristics}

Patient sampling

Primary objective: To prospectively determine the accuracy and the performance of a urinary cytokeratin 19 (UCYFRA 21-1) test for diagnosing endometriosis

Study population: Women undergoing laparoscopy for tubal ligation, chronic pelvic pain or infertility

Selection Criteria: Inclusion criteria: age 18 to 50; exclusion criteria: refusal to participate 
Lessey 2014 (Continued)

Study Design: Cross-sectional, two-gate design, prospective collection of samples, consecutive enrollment

Patient characteristics and Clinical presentation: Abdominal/pelvic pain or subfertility, request for tubal sterilisation setting

Age: Median age 36 years, range 21 to 45 years (controls); 32 years, range 18 to 44 years (endometriosis)

Number of participants enrolled: 98 women

Number of participants available for analysis: 98 women (cycle phase not reported)

Setting: University hospital, tertiary referral centre for endometriosis, Greenville Health System

Place of study: Greenville, South Carolina, USA

Period of study: January 2011 to April 2011

Language: English

Index tests

Index test: Urinary cytokeratin 19 (Cyfra 21-1)

Description of positive case definition by index test as reported: Two-step CYFRA 21-1 chemiluminescent immunoassay developed for a urine application (uCYFRA 21-1) on Abbott's ARCHITECT i2000SR analyser; detection range was 0.5 to $100 \mathrm{ng} / \mathrm{mL}$ based on a functional sensitivity of $0.5 \mathrm{ng} / \mathrm{ml}$

Threshold for positive result: For uCYFRA $21-1>5.3 \mathrm{ng} / \mathrm{mL}$ was chosen from the ROC curve (AUC = 0.53)

Examiners: Trained technician blinded to the clinical information and result of reference standard Interobserver variability: The inter- and intra-assay variability of UCYFRA $21-1$ assay was $<5 \%$

\section{Target condition and ref-} erence standard(s)
Target condition: Endometriosis

Prevalence of target condition in the sample: $n=63 / 98$ (64\%): stage I-II 50, stage III-IV 13; controls $n=$ 35

Reference standard: Laparoscopy and histology

Description of positive case definition by reference test as reported: Visual inspection confirmed by pathology; staging by rASRM classification

Examiners: Surgery performed in tertiary centre for endometriosis

Flow and timing

Time interval between index test and reference standard: The urinary samples were collected within 1 hour before laparoscopy

Withdrawals: None reported

Comparative

Key conclusions by the au- Despite the high specificity, the UCYFRA 21-1 has limited clinical value, either as a positive or as a negthors ative test. The non-significant differences between the distributions between endometriosis and controls and the non-informative AUC reveal the low practicability of the test in the clinical setting.
The authors declared no conflict of interests; supported by Abbott Laboratories and by Conselho Nacional de Desenvolvimento Científico e Tecnológico (CNPq) 240239/2012-1

\title{
Notes
}

\author{
For UCYFRA 21-1 there was no statistically significant difference between the groups, but diagnostic es- \\ timates were calculated and presented \\ Normalising the UCYFRA 21-1 results using urine creatinine or urine protein revealed similar AUC \\ uCYFRA 21-1 levels were not affected by severity of the disease
}


Lessey 2014 (Continued)

Cycle phase of testing was not reported

\section{Methodological quality}

\begin{tabular}{llll}
\hline Item & Authors' judgement & Risk of bias & Applicability concerns \\
\hline
\end{tabular}

\section{DOMAIN 1: Patient Selection}

Was a consecutive or ran- Yes

dom sample of patients

enrolled?

Did the study avoid inap- Yes

propriate exclusions?

Was a 'two-gate' design No

avoided?

\section{DOMAIN 2: Index Test All tests}

Were the index test re- Yes

sults interpreted without

knowledge of the results

of the reference standard?

\section{If a threshold was used, No}

was it pre-specified?

Was a cycle phase consid-

ered in interpretation of

No

the result of index test

\section{DOMAIN 3: Reference Standard}

Is the reference standards Yes

likely to correctly classify

the target condition?

\section{Were the reference stan-}

dard results interpreted

without knowledge of the

results of the index tests?

\begin{tabular}{lll}
\hline & Low & Low \\
\hline DOMAIN 4: Flow and Timing & &
\end{tabular}

Was there an appropriate Yes

interval between index

test and reference stan-

dard?

Did all patients receive the

same reference standard? 
Lessey 2014 (Continued)

Were all patients included Yes in the analysis?

Low

\section{Potlog-Naharia 2004}

\section{Study characteristics}

Patient sampling

Primary objective: To determine whether urine VEGF is elevated in women with endometriosis

Study population: Women who underwent laparoscopy for either pelvic pain or infertility

Selection criteria: Inclusion criteria: pre-menopausal age

Study design: Cross-sectional, single-gate design, prospective collection of samples

Patient characteristics and setting

Clinical presentation: Pelvic pain or infertility; 28 participants had past history of endometriosis

Age: Mean age $32.5 \pm 6.8$ years

Number of participants enrolled: 62 women

Number of participants available for analysis: 62 women ( 29 in follicular, 8 in peri-ovulatory and 22 in luteal cycle phase; for 3 women cycle phase was not reported)

Setting: Tertiary care public and private hospitals: Georgetown University Medical Center and the Clinical Centre of the National Institutes of Health

Place of study: Washington DC and Bethesda Maryland, USA

Period of study: 1998 to 2001

Language: English

Index tests

Index test: Urinary vascular endothelial growth factor (VEGF-A)

Details of the index test procedure as stated: Urinary VEGF-A was measured by ELISA assay (R\&D Systems Inc., Minneapolis, MN), detecting isoforms 121 and 165; VEGF concentrations were evaluated using both absolute values and those corrected for urinary creatinine values; sample handling described

Threshold for positive result: Not provided

Examiners: No information provided; unclear if blinded to the result of reference standard

Interobserver variability: Not reported

Target condition and reference standard(s)

\section{Target condition: Endometriosis}

Prevalence of target condition in the sample: $n=40 / 62(65 \%)$ : stage I-II 28, stage III-IV 12; controls $n=22$

Reference standard: Laparoscopy and histology

Description of positive case definition by reference standard as reported: Visual inspection, confirmed by histopathology; staging according to the rASRM classification

Examiners: Surgery performed by 2 operators ( 1 in each centre) 
Potlog-Naharia 2004 (Continued)

Flow and timing

Time interval between index test and reference standard: Urine sample was collected after induction of anaesthesia

Withdrawals: None

\section{Comparative}

Key conclusions by the authors

Conflict of interest None reported; supported by the Intramural Program, National Institute of Child Health and Human Development, Bethesda, Maryland

Notes

For VEGF there was no statistically significant difference between the groups - no data available for meta-analysis

Urinary VEGF levels were corrected for creatinine

Urinary VEGF levels were unaffected by severity of endometriosis or menstrual cycle phase

\section{Methodological quality}

\begin{tabular}{llll}
\hline Item & Authors' judgement & Risk of bias & Applicability concerns \\
\hline
\end{tabular}

DOMAIN 1: Patient Selection

\section{Was a consecutive or random Unclear}

sample of patients enrolled?

Did the study avoid inappro- Yes

priate exclusions?

Was a 'two-gate' design avoid- Yes

ed?

Unclear

Low

\section{DOMAIN 2: Index Test All tests}

\section{Were the index test results in- Unclear}

terpreted without knowledge

of the results of the reference

standard?

\section{If a threshold was used, was it No pre-specified?}

\section{Was a cycle phase considered Yes}

in interpretation of the result

of index test

\begin{tabular}{l}
\hline High \\
\hline DOMAIN 3: Reference Standard \\
\hline $\begin{array}{l}\text { Is the reference standards like- Yes } \\
\text { ly to correctly classify the tar- } \\
\text { get condition? }\end{array}$ \\
\hline
\end{tabular}


Potlog-Naharia 2004 (Continued)

Were the reference standard Yes results interpreted without knowledge of the results of the index tests?

\begin{tabular}{l}
\hline Low \\
\hline DOMAIN 4: Flow and Timing \\
\hline $\begin{array}{l}\text { Was there an appropriate in- } \\
\text { terval between index test and } \\
\text { reference standard? }\end{array}$ \\
$\begin{array}{l}\text { Did all patients receive the } \\
\text { same reference standard? }\end{array}$ \\
\hline $\begin{array}{l}\text { Were all patients included in } \\
\text { the analysis? }\end{array}$ \\
\hline
\end{tabular}

\section{Wang 2014}

\section{Study characteristics}

Patient sampling Primary objective: To detect endometriosis by urine peptide biomarkers using MALDI-TOF-MS and to identify interesting peptides using LC-MS/MS

Study population: Women who underwent laparoscopy for dysmenorrhoea, dyspareunia, chronic pelvic pain, ovarian cyst or infertility

Selection criteria: Exclusion criteria: oestrogen-dependent diseases or any prior hormonal treatment

Study design: Cross-sectional, single-gate design, prospective collection of samples

Patient characteristics and setting
Clinical presentation: Dysmenorrhoea, dyspareunia, chronic pelvic pain, ovarian cyst or infertility; all women had a regular cycle of 28 days

Age: Mean age $30.5 \pm 3.4$ years (endometriosis group), $31.5 \pm 4.2$ years (controls)

Number of participants enrolled: 147 women: 122 study cohort +25 validation cohort

Number of participants available for analysis: 147 women (105 in follicular and 42 luteal cycle phase)

Setting: Peking Union Medical College Hospital

Place of study: Beijing, China

Period of study: Not stated

Language: English

Index tests
Index test: Urinary proteome by MALDI-TOF-MS:

- 4 individual tests of single peptide: m/z 2052.3 Da, m/z 3393.9 Da, m/z 1579.2 Da [collagen alpha 6(IV) chain precursor] and $\mathrm{m} / \mathrm{z}$ 891.6 $\mathrm{Da}$ [collagen alpha 1 chain precursor]

- combined panel of five peptides m/z 1433.9 + 1599.4 + 2085.6 +6798.0 + 3217.2 Da 
Details of the index test procedure as stated: Mass spectra were acquired using a Reflex IV MALDI-TOFMS (Burker Daltonics) in positive linear mode; data analysis was performed using ClinProTools 2.2 software package13 (Bruker Daltonics, Bremen, Germany); 3 algorithms were used to set up a diagnostic model: generic (GA), decision tree (DTA) and quick classifier (QC) algorithms; sample processing described

Threshold for positive result: Not reported

Examiners: No information provided; unclear if blinded to the result of reference standard

Interobserver variability: Not reported

Target condition and reference standard(s)

Target condition: Endometriosis

Prevalence of target condition in the sample: $n=60 / 122(49 \%)$ : stage I-II 26, stage III-IV 34; controls $=62$ in the initial analysis; validation cohort: $n=11 / 25(44 \%)$ : stage I-II 5, stage III-IV 6; controls $n=14$

Reference standard: Laparoscopy

Description of positive case definition by reference standard as reported: Visual inspection using rAFS classification, not confirmed by histological examination

Examiners: No information provided

Flow and timing

Time interval between index test and reference standard: Urine sample was collected after induction of anaesthesia

Withdrawals: None

Comparative

Key conclusions by the authors
Women with endometriosis have a unique cluster of peptides in urine. Peptide proteomic profiling provides a novel method for non-invasive diagnosis of endometriosis.

\section{Conflict of interest None reported}

Notes

The authors identified a total of 36 peptides were significantly different between the endometriosis and control group, but diagnostic performance was assessed only for 4 individual peptides (3 down-regulated and 1 up-regulated in endometriosis) and for the combined diagnostic model

The estimates for the individual peptides are reported for the study population $(n=122)$; the estimates for the diagnostic model using panel of peptides were reported only for GA algorithm in a blind validation cohort $(n=25)$

Only 2 peptide peaks were identified: m/z 1579.2 Da (collagen alpha-6(IV) chain precursor) and m/z 891.6 Da (collagen alpha1 chain precursor)

No marked differences in the peptide pattern were detected between the follicular and the luteal phase.

\section{Methodological quality}

\begin{tabular}{llll}
\hline Item & Authors' judgement & Risk of bias & Applicability concerns
\end{tabular}

\section{DOMAIN 1: Patient Selection}

Was a consecutive or Unclear random sample of patients enrolled?

Did the study avoid in- Yes

appropriate exclusions? 
Wang 2014 (Continued)

Was a 'two-gate' design

Yes avoided?

Unclear

Low

\section{DOMAIN 2: Index Test All tests}

Were the index test re- Unclear

sults interpreted with-

out knowledge of the

results of the reference

standard?

\section{If a threshold was used, No}

was it pre-specified?

Was a cycle phase con- Yes

sidered in interpreta-

tion of the result of in-

dex test

\section{DOMAIN 3: Reference Standard}

Is the reference stan- Unclear
dards likely to correctly
classify the target con-
dition?

\section{Were the reference Yes}

standard results inter-

preted without knowl-

edge of the results of

the index tests?

\begin{tabular}{l}
\hline Unclear \\
\hline DOMAIN 4: Flow and Timing \\
\hline $\begin{array}{l}\text { Was there an appropri- Yes } \\
\text { ate interval between in- } \\
\text { dex test and reference } \\
\text { standard? }\end{array}$ \\
$\begin{array}{l}\text { Did all patients receive Yes } \\
\text { the same reference } \\
\text { standard? }\end{array}$ \\
$\begin{array}{l}\text { Were all patients in- } \\
\text { cluded in the analysis? }\end{array}$ \\
\hline
\end{tabular}


Yun 2014

\section{Study characteristics}

Patient sampling Primary objective: To validate and investigate the clinical value of urinary enolase I (NNE) in patients with endometriosis

Study population: Patients who underwent laparoscopy for diagnostic evaluation of pelvic masses, pelvic pain, suspicious endometriosis and infertility

Selection criteria: Exclusion criteria: postmenopausal status, previous use of hormone or gonadotropin-releasing hormone agonist, adenomyosis, endometrial cancer or hyperplasia, endometrial polyps, infectious diseases, chronic or acute inflammatory diseases, malignancy, autoimmune disease and cardiovascular disease

Study design: Cross-sectional, single-gate design, prospective collection of samples

Patient characteristics and setting
Clinical presentation: Pelvic masses, pelvic pain, suspicious endometriosis and infertility

Age: Mean age $31.48 \pm 6.30$ for endometriosis group, $29.35 \pm 6.87$ for control group

Number of participants enrolled: 59 women

Number of participants available for analysis: 59 women (in follicular or luteal cycle phase, numbers not specified; only moderate/severe endometriosis)

Setting: Gongnam Severance Hospital, Yonsei University College of Medicine

Place of study: Seoul, Republic of Korea

Period of study: January 2009 to December 2011

Language: English
Index test: Urinary enolase I (NNE-Cr)

Description of positive case definition by index test as reported: The concentration of urinary enolase I (NNE) was measured with commercial ELISAs according to the manufacturer's protocols (USCN Life Science \& Technology Company, TX) with minimal detectable concentration of $0.312 \mathrm{ng} / \mathrm{ml}$; urinary NNE values were normalised to urinary Cr concentrations; sample handling described

Threshold for positive result: NNE-Cr cutoff value was $>0.96 \mathrm{ng} / \mathrm{mgCr}$ using AUC 0.621 , not pre-specified

Examiners: No information provided, unclear if were blinded to the results of reference standard Interobserver variability: Not provided

\section{Target condition and ref- Target condition: Endometriosis} erence standard(s)

Reference standard: Laparoscopy and histology

Description of positive case definition by reference test as reported: Visual inspection confirmed on histopathology; staging according to the rASRM classification

Examiners: No information provided

Flow and timing

Time interval between index test and reference standard: Urine sample were collected after induction of anaesthesia

Withdrawals: No withdrawals reported 
Yun 2014 (Continued)

Key conclusions by the authors

Conflict of interest

The authors declared no conflict of interest; supported by the Basic Science Research Program through the National Research Foundation of Korea (NRF) funded by the Ministry of Education, Science and Technology (2010-0023323)

Notes

The absolute levels of urinary NNE did not significantly differ between women with and without endometriosis, but the difference was significant when NNE levels were corrected for Cr. The reported diagnostic estimates were calculated for NNE corrected for creatinine.

Urinary NNE levels, both absolute and normalised to $\mathrm{Cr}$, were unaffected by menstrual cycle phase.

Only information for severe disease available

\section{Methodological quality}

\begin{tabular}{llll}
\hline Item & Authors' judgement & Risk of bias & Applicability concerns
\end{tabular}

\section{DOMAIN 1: Patient Selection}

Was a consecutive or ran- Unclear

dom sample of patients

enrolled?

Did the study avoid inap- Yes

propriate exclusions?

Was a 'two-gate' design Yes

avoided?

\begin{tabular}{lll}
\hline & Unclear & High \\
\hline
\end{tabular}

\section{DOMAIN 2: Index Test All tests}

Were the index test re- Yes

sults interpreted without

knowledge of the results

of the reference stan-

dard?

If a threshold was used, No

was it pre-specified?

Was a cycle phase con-

sidered in interpretation

of the result of index test

\begin{tabular}{l} 
DOMAIN 3: Reference Standard \\
\hline $\begin{array}{l}\text { Is the reference stan- } \\
\text { dards likely to correctly } \\
\text { classify the target condi- } \\
\text { tion? }\end{array}$
\end{tabular}


Yun 2014 (Continued)

Were the reference standard results interpreted without knowledge of the results of the index tests?

\begin{tabular}{|c|c|c|}
\hline & Low & Low \\
\hline \multicolumn{3}{|c|}{ DOMAIN 4: Flow and Timing } \\
\hline $\begin{array}{l}\text { Was there an appropriate } \\
\text { interval between index } \\
\text { test and reference stan- } \\
\text { dard? }\end{array}$ & Yes & \\
\hline $\begin{array}{l}\text { Did all patients receive } \\
\text { the same reference stan- } \\
\text { dard? }\end{array}$ & Yes & \\
\hline \multirow[t]{2}{*}{$\begin{array}{l}\text { Were all patients includ- } \\
\text { ed in the analysis? }\end{array}$} & Yes & \\
\hline & Low & \\
\hline
\end{tabular}

Characteristics of excluded studies [ordered by study ID]

\begin{tabular}{ll}
\hline Study & Reason for exclusion \\
\hline Gjavotchanoff 2015 & $\begin{array}{l}\text { Study design outside inclusion criteria (retrospective collection of samples); insufficient diagnostic } \\
\text { test accuracy information (unable to construct } 2 \times 2 \text { tables) }\end{array}$ \\
\hline Itoh 2007 & $\begin{array}{l}\text { Study question outside inclusion criteria (focus on environmental exposure and risk of en- } \\
\text { dometriosis) }\end{array}$ \\
\hline Itoh 2008 & $\begin{array}{l}\text { Study question outside inclusion criteria (focus on environmental exposure and risk of en- } \\
\text { dometriosis) }\end{array}$ \\
\hline Itoh 2009 & $\begin{array}{l}\text { Study question outside inclusion criteria (focus on environmental exposure and risk of en- } \\
\text { dometriosis) }\end{array}$ \\
\hline Sharma 2010 & $\begin{array}{l}\text { Insufficient diagnostic test accuracy information (unable to construct } 2 \times 2 \text { tables) } \\
\text { Index test outside inclusion criteria (screening phase with qualitative biomarker evaluation, no } \\
\text { specific test defined and validated) }\end{array}$ \\
\hline
\end{tabular}

\section{Characteristics of studies awaiting classification [ordered by study ID]}

\section{Becker 2010}

\section{Study characteristics}

Patient sampling

Primary objective: To evaluate the clinical usefulness of urinary MMP in facilitating clinical decision-making or in monitoring therapeutic efficacy in patients with endometriosis. 
Study population: Patients who underwent laparoscopy for either infertility or pelvic pain.

Selection criteria: Exclusion criteria: history of malignancies, irritable bowel syndrome, autoimmune or infectious disease, or women using medical treatment for causes other than endometriosis

Study design: Cross-sectional, single-gate design, prospective collection of samples before surgery of during outpatient visits over the course of 3 years

Patient characteristics and setting
Clinical presentation: Pelvic pain and infertility

Age: Mean age 33 years (range 29 to 38 )

Number of participants enrolled: 1541 urinary samples collected

Number of participants available for analysis: 266 samples from 107 women obtained at different time points were randomly chosen for analysis; participants were classified into 4 groups depending on whether laparoscopic surgery was performed and endometriosis could be confirmed

Setting: Not reported

Place of study: Berlin, Germany

Period of study: Not recorded

Language: English

Index tests

Description of positive case definition by index test as reported: Zymograms were conducted using an established procedure. Different MMPs were distinguished from each other according to their molecular weight and confirmed by immunoblot analysis. Correlating MMP expression to creatinine clearance did not have an impact on the results.

Threshold for positive result: Not reported

Examiners: Two examiners were blinded to the clinical status of the patients

Interobserver variability: Not provided

Target condition dard(s) and reference stan-

\section{Target condition: Endometriosis}

Prevalence of target condition in the sample: Not reported

Reference standard: Laparoscopy and histology (not all patients received laparoscopy; numbers not reported)

Description of positive case definition by reference test as reported: Visual inspection confirmed on histopathology; staging according to the rASRM classification

Examiners: Surgeries performed by experienced surgeons who were blinded to the MMP data

Flow and timing Time interval between index test and reference standard: Urine samples were collected before surgery (catheter) if surgery was performed and if available during outpatient visits (midstream) over the course of 3 years.

Withdrawals: Not reported

\section{Comparative}

Notes

Data to construct $2 \times 2$ not presented. A subgroup of the data set may be relevant to the review question, but need further clarification with the authors. 
Tokushige 2011

\section{Study characteristics}

Patient sampling

Primary objective: To investigate whether proteins secreted in urine differ between women with and without endometriosis.

Study population: Women with and without endometriosis undergoing laparoscopy

Selection criteria: Exclusion criteria: medical therapy for at least 3 months before surgery

Study design: Cross-sectional, appeared to be retrospective collection of urinary samples after diagnosis of endometriosis was made

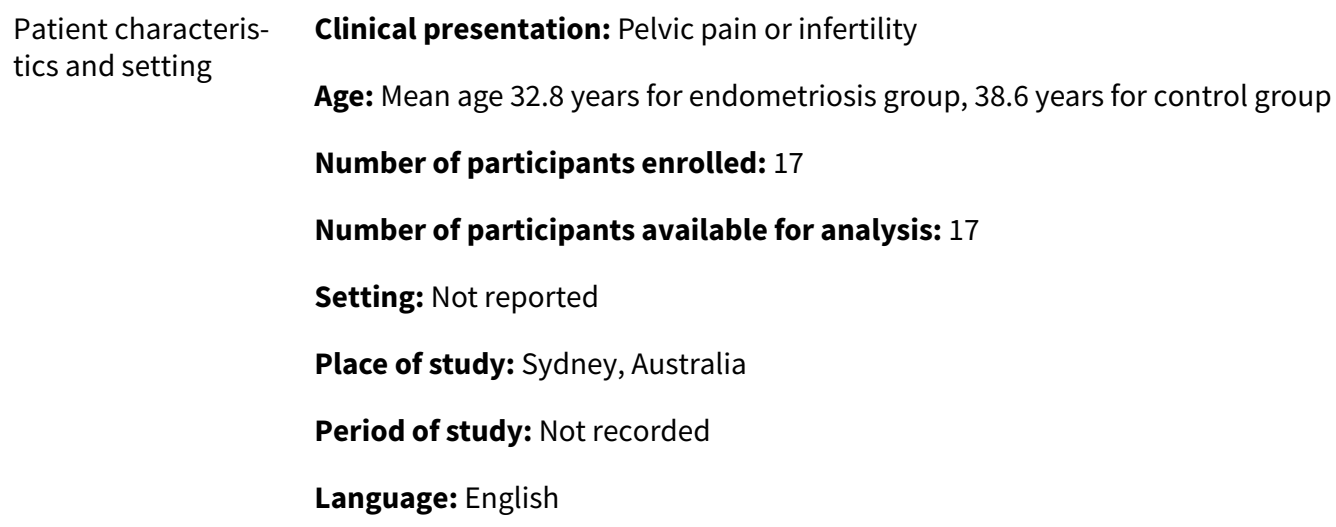

Description of positive case definition by index test as reported: Mass spectra were acquired across a $\mathrm{m} /$ z range of 840 to 3500 using a Voyager DE STR (Applied Biosystems, Fostr City, CA). The samples were blotted with the CK 19 antibody (Millipore).

Threshold for positive result: Not reported

Examiners: Not reported

Interobserver variability: Not provided

Target condition and reference stan$\operatorname{dard}(\mathrm{s})$
Target condition: Endometriosis

Prevalence of target condition in the sample: $6 / 11$

Reference standard: Laparoscopy and histology

Description of positive case definition by reference test as reported: Visual inspection confirmed on histopathology; staging according to the rASRM classification

Examiners: Not reported

Time interval between index test and reference standard: Urine samples appeared to be collected after the laparoscopy

Withdrawals: Not reported

$$
\text { Comparative }
$$

Notes Appears to be retrospective collection of urine samples. Clarification from authors required.


Characteristics of ongoing studies [ordered by study ID]

\section{NCT02337816}

Trial name or title

Role of Metabolomics in the Diagnosis of Endometriosis

ClinicalTrials.gov Identifier: NCT02337816

Other study name: ENDOMETAB01

Target condition and reference standard(s) dometriosis
Objective: To identify an alteration in the expression of the metabolites in women with en-

Primary outcome measures: Plasma and urine concentration of metabolites [Time Frame: At least one month after discontinuation of hormonal therapies and before laparoscopic surgery]

Study design: Non-randomised, parallel assignment, open label

Target condition: Endometriosis

Reference standard: Laparoscopy + histopathology

\begin{tabular}{ll}
\hline Index and comparator tests & Urine and blood \\
\hline Starting date & December 2014 \\
\hline Contact information & Responsible party: Stefano Angioni, University of Cagliari \\
\hline Notes & Current status - ongoing, but not recruiting participants \\
\hline
\end{tabular}

\section{A T A}

Presented below are all the data for all of the tests entered into the review.

Table Tests. Data tables by test

\begin{tabular}{|c|c|c|}
\hline Test & No. of studies & No. of participants \\
\hline $1 \mathrm{NNE}-\mathrm{Cr}(>0.96 \mathrm{ng} / \mathrm{mgCr})$ & 1 & 59 \\
\hline 2 VDBP-Cr (> $87.83 \mathrm{ng} / \mathrm{mgCr})$ & 1 & 95 \\
\hline 3 Proteome by MALDI-TOF-MS (peptide m/z 1824.3 Da; $\geq 29.34 \mathrm{au}$ ) & 1 & 28 \\
\hline 4 Proteome by MALDI-TOF-MS (peptide m/z 1767.1 Da; $\geq 35.22 \mathrm{au}$ ) & 1 & 27 \\
\hline 5 Proteome by MALDI-TOF-MS (peptide m/z 2052.3 Da; cut-off not reported) & 1 & 122 \\
\hline 6 Proteome by MALDI-TOF-MS (peptide m/z 3393.9 Da; cut-off not reported) & 1 & 122 \\
\hline $\begin{array}{l}7 \text { Proteome by MALDI-TOF-MS (peptide m/z 1579.2 Da [collagen alpha 6(IV) } \\
\text { chain precursor]; cut-off not reported) }\end{array}$ & 1 & 122 \\
\hline $\begin{array}{l}8 \text { Proteome by MALDI-TOF-MS (peptide m/z } 891.6 \text { Da [collagen alpha1 chain } \\
\text { precursor];; cut-off not reported) }\end{array}$ & 1 & 122 \\
\hline
\end{tabular}


9 Proteome by MALDI-TOF-MS ( 5 peptides m/z 1433.9 +1599.4 + 2085.6+6798.0 1

Test 1. NNE-Cr $(>0.96 \mathrm{ng} / \mathrm{mgCr})$.

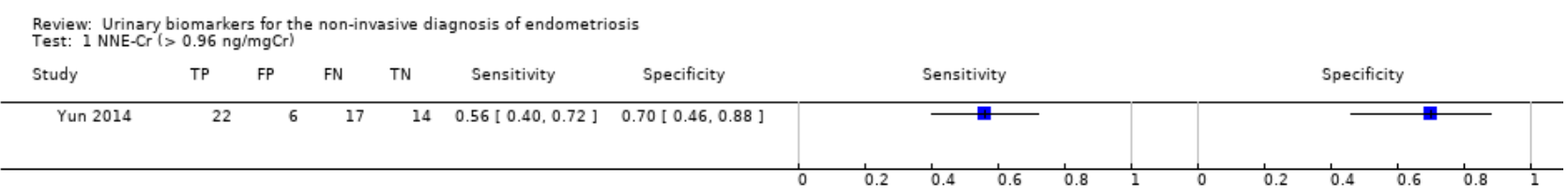

Test 2. VDBP-Cr (> 87.83 ng/mgCr).

Review: Urinary biomarkers for the non-invasive diagnosis of endometriosis

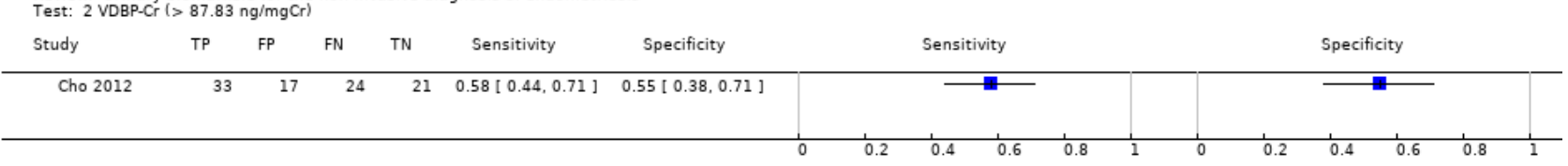

Test 3. Proteome by MALDI-TOF-MS (peptide m/z 1824.3 Da; $\geq 29.34 \mathrm{au}$ ).

Review: Urinary biomarkers for the non-invasive diagnosis of endometriosis
Test: 3 Proteome by MALDI-TOF-MS (peptide $\mathrm{m} / 2$ 1824.3 Da: $\geq 29.34$ au)

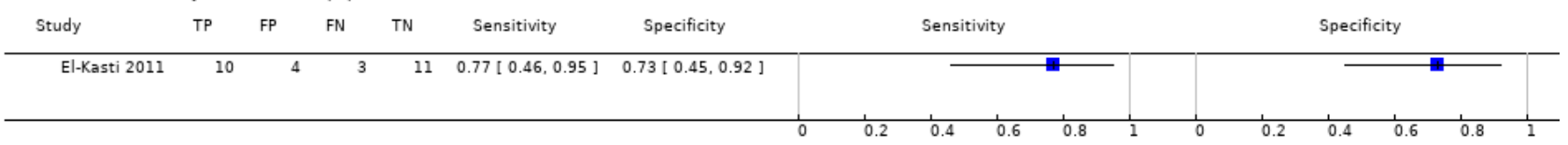

Test 4. Proteome by MALDI-TOF-MS (peptide m/z 1767.1 Da; $\geq 35.22 \mathrm{au}$ ).

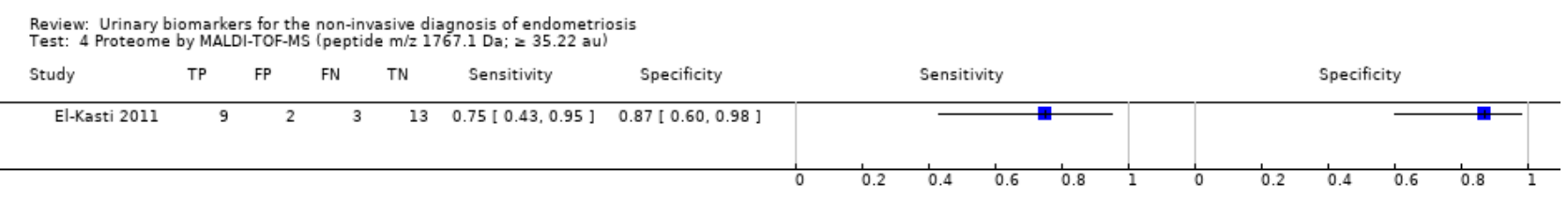

Test 5. Proteome by MALDI-TOF-MS (peptide m/z 2052.3 Da; cut-off not reported).

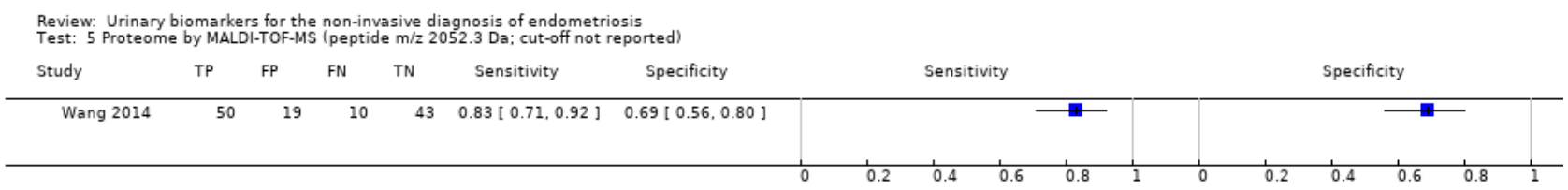


Test 6. Proteome by MALDI-TOF-MS (peptide m/z 3393.9 Da; cut-off not reported).

Review: Urinary biomarkers for the non-invasive diagnosis of endometriosis
Test: 6 Proteome by MALDI-TOF-MS (peptide $\mathrm{m} / 2$ 3393.9 Da: cut-off not reported

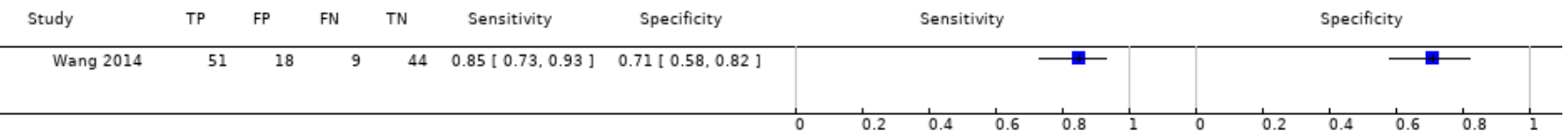

Test 7. Proteome by MALDI-TOF-MS (peptide $\mathrm{m} / \mathrm{z}$ 1579.2 Da

[collagen alpha 6(IV) chain precursor]; cut-off not reported).

Review: Urinary biomarkers for the non-invasive diagnosis of endometriosis
Test: 7 Proteome by MALDI-TOF-MS (peptide $\mathrm{m} / 2$ 1579.2 Da [collagen alpha 6 (IV) chain precursor]; cut-off not reported)

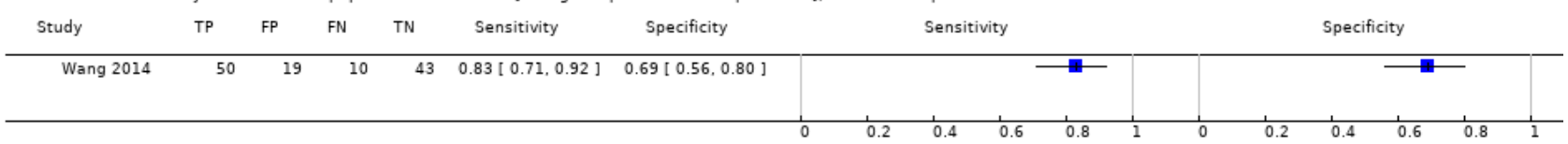

Test 8. Proteome by MALDI-TOF-MS (peptide m/z 891.6 Da [collagen alpha1 chain precursor];; cut-off not reported).

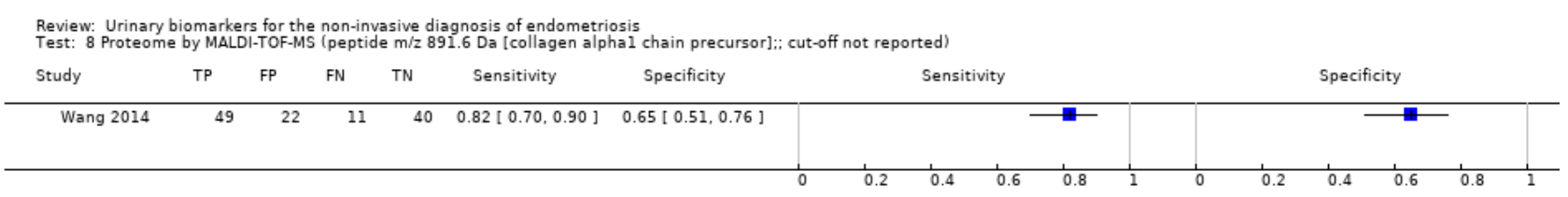

Test 9. Proteome by MALDI-TOF-MS (5 peptides $\mathrm{m} / \mathrm{z} 1433.9$

+1599.4 + 2085.6 + 6798.0 + 3217.2 Da; cut-off not reported).

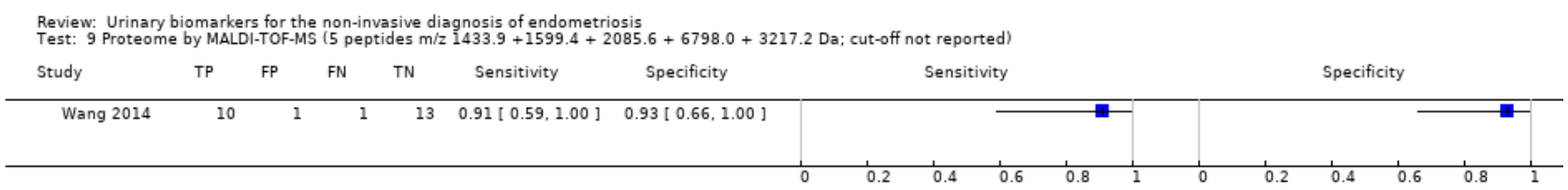

Test 10. CK 19 [CYFRA 21-1] (>5.3 ng/ml).

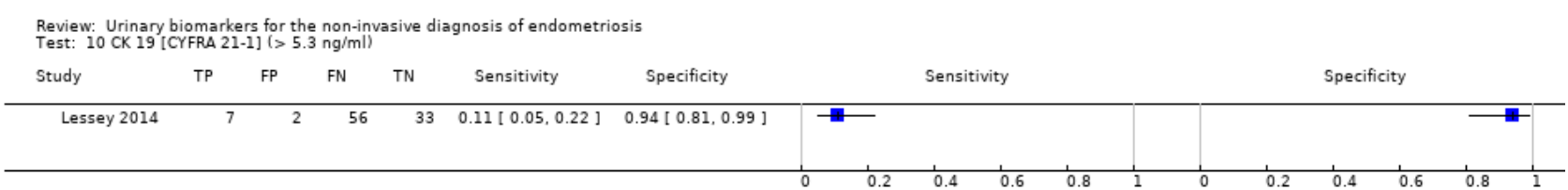

\section{ADDITIONAL TABLES}


Table 1. Staging of endometriosis, rASRM classification

\begin{tabular}{|c|c|c|c|c|}
\hline \multirow[t]{3}{*}{ Peritoneum } & Endometriosis & $<1 \mathrm{~cm}$ & 1 to $3 \mathrm{~cm}$ & $>3 \mathrm{~cm}$ \\
\hline & Superficial & 1 & 2 & 4 \\
\hline & Deep & 2 & 4 & 6 \\
\hline \multirow[t]{6}{*}{ Ovary } & R Superficial & 1 & 2 & 4 \\
\hline & Deep & 4 & 16 & 20 \\
\hline & L Superficial & 1 & 2 & 4 \\
\hline & Deep & 4 & 16 & 20 \\
\hline & Posterior Cul-de-sac Obliteration & Partial & Complete & \\
\hline & & 4 & 40 & \\
\hline \multirow[t]{5}{*}{ Ovary } & Adhesions & $<1 / 3$ Enclosure & $\begin{array}{l}\text { 1/3-2/3 Enclo- } \\
\text { sure }\end{array}$ & $>2 / 3$ Enclosure \\
\hline & R Filmy & 1 & 2 & 4 \\
\hline & Dense & 4 & 8 & 16 \\
\hline & L Filmy & 1 & 2 & 4 \\
\hline & Dense & 4 & 8 & 16 \\
\hline \multirow[t]{4}{*}{ Tube } & R Filmy & 1 & 2 & 4 \\
\hline & Dense & $4^{*}$ & $8^{*}$ & 16 \\
\hline & L Filmy & 1 & 2 & 4 \\
\hline & Dense & $4^{\star}$ & $8^{\star}$ & 16 \\
\hline
\end{tabular}

* If the fimbriated end of the fallopian tube is completely enclosed, change the point assignment to 16

American Society for Reproductive Medicine 1997

Table 2. Urinary biomarkers for endometriosis

\section{Angiogenesis/Growth factors and their receptors}

VEGF-A (vascular endothelial growth factor - A $)^{1}$

sFlt-1 [sVEGFR-1] (soluble fms-like tyrosine kinase or variant of VEGF receptor 1$)^{2}$

\section{Cell adhesion molecules and other matrix-related proteins}

MMP-2 (matrix metalloproteinase-2)2

MMP-9 (matrix metalloproteinase-9)2 
Table 2. Urinary biomarkers for endometriosis (Continued)

MMP-9/ NGAL (matrix metalloproteinase-9/neutrophil gelatinase-associated lipocalin)2

\section{Cytokines}

TNF-alpha (tumour necrosis factor alfa) ${ }^{1}$

\section{Cytoskeleton molecules}

CK-19 or CYFRA 21-1 (Cytokeratin-19) ${ }^{1}$

\section{High throughput markers}

Proteome

\section{Oxidative stress markers}

8-iso-PGF2a (8-iso-prostaglandin F2a) ${ }^{2}$

\section{Other Peptides/proteins}

VDBP (vitamin D binding protein)

NNE (enolase I)

Collagen precursors

\section{Prealbumin 2}

Alpha 1 antitrypsin 2

Chain A solution structure of $\mathrm{Bb}^{\prime}$ domains of human protein disulfide isomerase ${ }^{2}$

1 Urinary biomarkers that did not exhibit differential expression in endometriosis

2 Urinary biomarkers that exhibited differential expression in endometriosis, but for which the diagnostic estimates were not available

Table 3. Application of the QUADAS-2 tool for assessment of methodological quality of the included studies

\begin{tabular}{ll}
\hline Domain 1 - Patient selection & \\
\hline Description & Describe methods of patient selection and included patients \\
\hline Type of bias assessed & Selection bias, spectrum bias \\
\hline Review Question & $\begin{array}{l}\text { Women of reproductive age with clinically suspected endometriosis (symptoms, clinical examina- } \\
\text { tion } \pm \text { presence of pelvic mass), scheduled for surgical exploration of pelvic/abdominal cavity for } \\
\text { confirmation of the diagnosis } \pm \text { treatment }\end{array}$ \\
\hline Informaton collected & $\begin{array}{l}\text { Study objectives, study population, selection (inclusion and exclusion criteria), study design, clin- } \\
\text { ical presentation, age, number of participants enrolled and number of participants available for } \\
\text { analysis, setting, place and period of the study }\end{array}$ \\
\hline
\end{tabular}

\section{Signalling question 1}

Was a consecutive or random sample of patients enrolled? 
Table 3. Application of the QUADAS-2 tool for assessment of methodological quality of the included studies (Continued)

Yes

If a consecutive sample or a random sample of the eligible patients was included in the study

No

Unclear

If non-consecutive sample or non-random sample of the eligible patients was included in the study

\author{
If this information was unclear
}

\section{Signalling question 2}

Yes included, with an exception for those who a) had a history of medical conditions or were on medical therapy that would have potentially interfered with interpretation of index test (e.g. malignancy, pregnancy, autoimmune disorders, infectious diseases, treatment with hormonal or immunomodulator substances); b) refused to participate in the study; or c) were unfit for surgery

No

If the study excluded the patients based on education level, psychosocial factors, genetic testing or phenotype or excluded patients with any co-morbidities commonly present in general population, including a population that could have undergone a testing for endometriosis in clinical setting (hypertension, asthma, obesity, benign gastro-intestinal or renal disease, etc)

Unclear If the study did not provide clear definition of the selection (inclusion or exclusion) criteria and 'no' judgement was not applicable

\begin{tabular}{ll} 
Signalling question 3 & Was a 'two-gate' design avoided? \\
\hline Yes & $\begin{array}{l}\text { If the study had a single set of inclusion criteria, defined by the clinical presentation (i.e. only par- } \\
\text { ticipants in whom the target condition is suspected) - a 'single-gate' study design }\end{array}$ \\
\hline No & $\begin{array}{l}\text { If the study had more than one set of inclusion criteria in respect to clinical presentation (i.e. partic- } \\
\text { ipants suspected of target condition and participants with alternative diagnosis in whom the target } \\
\text { condition would not be suspected in clinical practice) - a 'two-gate' study design }\end{array}$
\end{tabular}

Unclear If it was unclear whether a 'two-gate deign' was avoided or not

Risk of bias Could the selection of patients have introduced bias?

\begin{tabular}{|c|c|}
\hline Low & If 'yes' classification for all the above 3 questions \\
\hline High & If 'no' classification for any of the above 3 questions \\
\hline Unclear & If 'unclear' classification for 3 of the above questions and 'high risk' judgement was not applicable \\
\hline Concerns about applicability & Are there concerns that the included patients do not match the review question? \\
\hline Low & $\begin{array}{l}\text { If the study includes only clinically relevant population that would have undergone index test in re- } \\
\text { al practice and includes representative form of target condition }\end{array}$ \\
\hline High & $\begin{array}{l}\text { If the study population differed from the population defined in the review question in terms of de- } \\
\text { mographic features and co-morbidity (e.g. studies with multiple sets of inclusion criteria with re- } \\
\text { spect to clinical presentation including either healthy controls or alternative diagnosis controls } \\
\text { that would not have undergone index test in real practice). Further, if target condition diagnosed } \\
\text { in the study population was not representative of the entire spectrum of disease, such as limited } \\
\text { spectrum of severity (e.g. only mild forms) or limited type of endometriosis (e.g. only DIE) }\end{array}$ \\
\hline Unclear & If this information was unclear (e.g. severity of endometriosis was not reported) \\
\hline
\end{tabular}

\section{Domain 2 - Index test}


Table 3. Application of the QUADAS-2 tool for assessment of methodological quality of the included studies (Continued)

Description

Type of bias assessed

Review Question

Informaton collected

Signalling question 1

Yes

Yes

No

No

Unclear

\begin{tabular}{l} 
Signalling question 2 \\
\hline Yes \\
\hline No \\
\hline Unclear
\end{tabular}

Signalling question 3

$\begin{array}{ll}\text { Yes } & \begin{array}{l}\text { If all the included participants were in the same phase of menstrual cycle or if the study reported } \\ \text { subgroup analyses per cycle phase or if study reported the pooled estimates after impact of the cy- } \\ \text { cle phase on biomarker expression was not detected }\end{array}\end{array}$

No

If study included participants in different phases of menstrual cycle, but effect of cycle phase on index test was not assessed

\begin{tabular}{ll}
\hline Unclear & If the cycle phase was not reported \\
\hline Risk of bias & Could the conduct or interpretation of the index test have introduced bias? \\
\hline Low & If 'yes' classification for all the above 3 questions \\
\hline High & If 'no' classification for any of the above 3 questions \\
\hline Unclear & $\begin{array}{l}\text { If 'unclear' classification for any of the above } 3 \text { questions and 'high risk' judgement was not applic- } \\
\text { able }\end{array}$ \\
\hline Concerns about applicability & $\begin{array}{l}\text { Are there concerns that the index test, its conduct, or interpretation differ from the review } \\
\text { question? }\end{array}$ \\
\hline Low & $\begin{array}{l}\text { We considered all types of urinary biomarkers as eligible; therefore all the included studies were } \\
\text { classified as 'low concern', unless 'unclear' judgement was applicable }\end{array}$ \\
\hline
\end{tabular}


Table 3. Application of the QUADAS-2 tool for assessment of methodological quality of the included studies (Continued)

High
We did not consider the studies where index tests other than urinary biomarkers were included (or excluded information on other index tests reported in addition to urine tests) or where index test looked at other target conditions not specified in the review (e.g. studies aimed at classifying pelvic masses as benign and malignant); therefore none of the included studies was classified as 'high concern'
If study did not present sufficient information on at least one of the following: laboratory method, sample handling, reagents used, experience of the test operators

\section{Domain 3 - Reference standard}

\begin{tabular}{ll}
\hline Description & Describe the reference standard, how it was conducted and interpreted \\
\hline Type of bias assessed & Verification bias, bias in estimation of diagnostic accuracy due to inadequate reference standard \\
\hline Review Question & $\begin{array}{l}\text { Target condition - pelvic endometriosis, ovarian endometriosis, DIE. Reference standard - visuali- } \\
\text { sation of endometriosis at surgery (laparoscopy or laparotomy) with or without histological confir- } \\
\text { mation }\end{array}$ \\
\hline Informaton collected & $\begin{array}{l}\text { Target condition, prevalence of target condition in the sample, reference standard, description of } \\
\text { positive case definition by reference test as reported, examiners (number, level of expertise, blind- } \\
\text { ing) }\end{array}$
\end{tabular}

\section{Signalling question 1}

Yes

\section{Were the reference standards likely to correctly classify the target condition?}

If the study reported at least one of the following: surgical procedure was described in sufficient details; or criteria for positive reference standard were stated; or diagnosis was confirmed by histopathology; or the procedure was performed by the team with high level of expertise in diagnosis/surgical treatment of target condition, including tertiary referral centres for endometriosis

\begin{tabular}{|c|c|}
\hline No & $\begin{array}{l}\text { If reference standard did not classify target condition correctly; considering the inclusion criteria } \\
\text { and a nature of the reference standard, none of the studies were classified as 'no' for this item }\end{array}$ \\
\hline Unclear & If information on execution of the reference standard, its interpretation or operators was unclear \\
\hline Signalling question 2 & $\begin{array}{l}\text { Were the reference standard results interpreted without knowledge of the results of the index } \\
\text { tests? }\end{array}$ \\
\hline Yes & If operators performing the reference test were unaware of the results of index test \\
\hline No & If operators performing the reference test were aware of the results of index test \\
\hline Unclear & If this information was unclear \\
\hline Risk of bias & Could the reference standard, its conduct, or its interpretation have introduced bias? \\
\hline Low & If 'yes' classification for all the above 2 questions \\
\hline High & If 'no' classification for any of the above 2 questions \\
\hline Unclear & $\begin{array}{l}\text { If 'unclear' classification for any of the above } 2 \text { questions and 'high risk' judgement was not applic- } \\
\text { able }\end{array}$ \\
\hline Concerns about applicability & $\begin{array}{l}\text { Are there concerns that the target condition as defined by the reference standard does not } \\
\text { match the question? }\end{array}$ \\
\hline
\end{tabular}


Table 3. Application of the QUADAS-2 tool for assessment of methodological quality of the included studies (Continued)

Low

Considering the inclusion criteria, all the studies were classified as 'low concern', therefore all the included studies were classified as 'low concern'

\begin{tabular}{ll} 
High & $\begin{array}{l}\text { We excluded the studies where participants did not undergo surgery for diagnosis of endometrio- } \\
\text { sis, therefore none of the included studies were classified as 'high concern' }\end{array}$ \\
\hline Unclear & $\begin{array}{l}\text { Only studies were laparoscopy/laparotomy served as a reference test were included; therefore } \\
\text { none of the included studies was classified as 'unclear concern' }\end{array}$ \\
\hline Domain 4 - Flow and timing & $\begin{array}{l}\text { Describe any patients who did not receive the index tests or reference standard or who were ex- } \\
\text { cluded from the } 2 \times 2 \text { table, describe the interval and any interventions between index tests (sam- } \\
\text { ple collection) and the reference standard }\end{array}$ \\
\hline Description &
\end{tabular}

\begin{tabular}{ll} 
Type of bias assessed & Disease progression bias, bias of diagnostic performance due to missing data \\
\hline Review Question & $\begin{array}{l}\text { Less than } 12 \text { months interval between index test (sample collection) and reference standard - en- } \\
\text { dometriosis may progress over time, so we had chosen an arbitrary time interval of } 12 \text { months as } \\
\text { an acceptable time interval between the sample collection and surgical confirmation of diagnosis }\end{array}$ \\
\hline Informaton collected & $\begin{array}{l}\text { Time interval between index test (sample collection) and reference standard, withdrawals (overall } \\
\text { number reported and if were explained) }\end{array}$
\end{tabular}

\begin{tabular}{ll}
\hline Signalling question 1 & $\begin{array}{l}\text { Was there an appropriate interval between index test (sample collection) and reference stan- } \\
\text { dard? }\end{array}$ \\
\hline Yes & If time interval was reported and was less than 12 months \\
\hline No & $\begin{array}{l}\text { We excluded all the studies where time interval was longer than } 12 \text { months, therefore none of the } \\
\text { included studies were classified as 'no' for this item }\end{array}$ \\
\hline Unclear & $\begin{array}{l}\text { If time interval was not stated clearly, but authors' description allowed to assume that the interval } \\
\text { was reasonably short }\end{array}$
\end{tabular}

\begin{tabular}{ll}
\hline Signalling question 2 & Did all women receive the same reference standard? \\
\hline Yes & $\begin{array}{l}\text { If all participants underwent laparoscopy or laparotomy as a reference standard; considering the } \\
\text { inclusion criteria, all the studies were classified as 'yes' for this item, as anticipated }\end{array}$ \\
\hline No & $\begin{array}{l}\text { If all participants did not undergo surgery or had alternative reference standard or if only a subset } \\
\text { of participants had surgery as reference standard, but the information on this population was not } \\
\text { available in isolation; considering the inclusion criteria, none of the included studies were classi- } \\
\text { fied as 'no' for this item }\end{array}$
\end{tabular}

\begin{tabular}{ll}
\hline Unclear & $\begin{array}{l}\text { If this information was unclear; considering the inclusion criteria, none of the included studies } \\
\text { were classified as 'unclear' for this item }\end{array}$ \\
\hline Signalling question 3 & Were all women included in the analysis? \\
\hline Yes & $\begin{array}{l}\text { If all the women were included in the analysis or if women were excluded because they did not } \\
\text { meet inclusion criteria prior to execution of index test or if the withdrawals were less than } 5 \% \text { of } \\
\text { the enrolled population (arbitrary selected cut-off) }\end{array}$ \\
\hline No & $\begin{array}{l}\text { If any patients were excluded from the analysis because of un interpretable results, inability to un- } \\
\text { dergo either index test or reference standard or for unclear reasons }\end{array}$ \\
\hline
\end{tabular}


Table 3. Application of the QUADAS-2 tool for assessment of methodological quality of the included studies (Continued) Unclear If this information was unclear

\begin{tabular}{ll}
\hline Risk of bias & Could the patient flow have introduced bias? \\
\hline Low & If 'yes' classification for all the above 3 questions \\
\hline High & If 'no' classification for any of the above 3 questions \\
\hline Unclear & $\begin{array}{l}\text { If 'unclear' classification for any of the above } 3 \text { questions and 'high risk' judgement was not applic- } \\
\text { able }\end{array}$ \\
\hline
\end{tabular}

\section{AP PEN DICES}

\section{Appendix 1. Search strategy for MEDLINE (OVID platform)}

Database: MEDLINE (Ovid) <1946 to February, week 22015 (16.2.2015)>

Ovid MEDLINE(R) In-Process \& Other Non-Indexed Citations, Ovid MEDLINE(R) Daily and Ovid MEDLINE(R) Search Strategy:

1 (biomarker\$ or marker\$).tw. (605002)

2 Laboratory Test\$.tw. (29839)

3 growth factor\$.tw. (272049)

4 scatter factor\$.tw. (1287)

5 cytokine\$.tw. (250618)

6 hepatocyte growth factor.tw. (8053)

7 (FGF or fibroblast growth factor\$).tw. (31798)

8 (PDGF or platelet derived growth factor\$).tw. (19864)

9 (EGF or epidermal growth factor\$).tw. (58069)

10 (IGF-I or insulin-like growth factor\$ or IGF1).tw. (43539)

11 (TGF-a or transforming growth factor alfa or TGFa).tw. (281)

12 (TGF-b or transforming growth factor beta or TGFb).tw. (28842)

13 (EGFR or epidermal growth factor receptor\$).tw. (41719)

14 (VEGF or vascular endothelial growth factor\$).tw. (53588)

15 exp Luteinizing Hormone/bl [Blood] (24587)

16 leptin\$.tw. (24994)

17 exp Progesterone/bl [Blood] (18412)

18 Proteolytic enzyme\$.tw. (9768)

19 exp matrix metalloproteinase 1 / or exp matrix metalloproteinase 2/ or exp matrix metalloproteinase 3 / or exp matrix metalloproteinase 9/ (22968) 
20 matrix metalloproteinase\$.tw. (34522)

21 MMP\$.tw. (44439)

22 TIMP\$.tw. (10777)

23 exp "tissue inhibitor of metalloproteinase-1"/ or exp "tissue inhibitor of metalloproteinase-2"/ (6146)

24 exp Glycoproteins/ (637149)

25 (Ca-125 or Ca125 or cancer antigen 125).tw. (6761)

26 (Ca-19-9 or Ca19-9 or cancer antigen 19-9).tw. (4194)

27 (PP 14 or PP14).tw. (229)

28 serum placental protein\$.tw. (33)

29 exp Follistatin/ (1134)

30 Osteopontin\$.tw. (6769)

31 exp intercellular adhesion molecule-1/ or exp selectins/ (25302)

32 soluble intercellular adhesion.tw. (1588)

33 Soluble adhesion molecule\$.tw. (779)

34 sICAM.tw. (2258)

35 sVCAM\$.tw. (1277)

36 (sEcadherin or soluble E-cadherin).tw. (95)

37 (sEselectin or soluble E-selectin).tw. (689)

38 exp t-lymphocytes/ or exp natural killer t-cells/ (272580)

39 Immune cells alteration\$.tw. (1)

40 (T helper\$ or T supressor\$ or T helper\$ T supressor\$ ratio).tw. (21275)

41 Total complement level\$̣.tw. (23)

42 Autoantibodies.tw. (33457)

43 exp Antibodies, Antiphospholipid/ (7522)

44 Anti-endometrial.tw. (23)

45 Antiphospholipid\$.tw. (9974)

46 exp hla antigens/ or exp hla-a1 antigen/ or exp hla-a2 antigen/ (64462)

47 (HLA or human leucocyte antigen\$).tw. (80501)

48 Anti-laminin-1.tw. (33)

49 Anti-thyroid.tw. (1414)

50 Anti-Thomsen Friedenreich antigen\$.tw. (6)

51 Anti-transferrin.tw. (275)

52 Anti-LDL.tw. (181)

53 (Anti-2HSG or Heremans-Schmidt glycoprotein).tw. (3)

54 interleukin\$.tw. (175195) 
55 (MCP-I or monocyte chemoattractant protein-I).tw. (44)

56 (MIF or migration inhibitory factor\$).tw. (4479)

57 (TNF-a or tumour necrosis factor\$ alfa).tw. (1344)

58 Fas ligand\$.tw. (6032)

59 Endometrial marker\$.tw. (11)

60 CAMs.tw. (1756)

61 cell adhesion molecule\$.tw. (20903)

62 exp Integrins/ (44414)

63 Integrin\$.tw. (39960)

64 Selectin\$.tw. (55426)

65 Cadherin\$.tw. (20780)

66 Aromatase P450.tw. (180)

67 estrogen receptor\$.tw. (38819)

68 progesterone receptor\$.tw. (16623)

69 MTMMP\$.tw. (7)

70 cyr61.tw. (559)

71 exp Cysteine-Rich Protein 61/ (386)

72 cysteine-rich heparin-binding protein\$.tw. (9)

73 (ANXA 1 or ANXA1).tw. (313)

74 (Annexin 1 or Annexin1).tw. (339)

75 (PGP 9?5 or PGP9?5 or protein gene product\$).tw. (2096)

76 serum marker\$.tw. (5429)

77 neural marker\$.tw. (925)

78 cell surface marker\$.tw. (4456)

79 inflammatory marker\$.tw. (10916)

80 microarray\$.tw. (75404)

81 microRNA\$..tw. (29731)

82 proteomic\$.tw. (45292)

83 genomic\$.tw. (190985)

84 (endometri\$ adj2 biops\$).tw. (3411)

85 Follistatin\$.tw. (1663)

86 Vascular Endothelial Growth Factor A/ (35738)

87 Vitamin D-Binding Protein/ (1282)

88 exp Cytokines/ (547522)

89 exp interleukins/ or exp interleukin-1/ or exp interleukin-6/ or exp interleukin-8/ or exp interleukin-12/ or exp interleukin-13/ (188479) 
90 exp Epidermal Growth Factor/ (21298)

91 exp Fibroblast Growth Factors/ (25075)

92 Platelet-Derived Growth Factor/ (11030)

93 Keratin-19/ (1090)

$94 \exp$ Clinical Laboratory Techniques/ (2132820)

95 (Luteinizing Hormone\$ or LH).tw. (56679)

96 cytokeratin-19.tw. (1469)

97 (VDBP or vitamin D-binding protein\$).tw. (1158)

98 urinary peptide\$.tw. (137)

99 VDBP-Cr.tw. (1)

100 urinary VDBP corrected for creatinine expression.tw. (1)

101 urinary marker\$.tw. (638)

102 or/1-101 (4086291)

103 Endometriosis/di [Diagnosis] (3354)

104102 or 103 (4088946)

105 exp Endometriosis/ (17244)

106 Endometrio\$.tw. (21492)

107105 or $106(24940)$

108104 and 107 (10490)

109 (animals not (humans and animals)).sh. (3892900)

110108 not $109(10113)$

\section{Additional search February 2015 - May 2015}

Ovid MEDLINE(R) In-Process \& Other Non-Indexed Citations, Ovid MEDLINE(R) Daily and Ovid MEDLINE(R) <1946 to Present (3.9.2015)> Search Strategy:

1 (biomarker\$ or marker\$).tw. (652345)

2 Laboratory Test\$̣.tw. (31389)

3 growth factor\$.tw. (287701)

4 scatter factor\$.tw. (1326)

5 cytokine\$.tw. (267766)

6 hepatocyte growth factor.tw. (8585)

7 (FGF or fibroblast growth factor\$).tw. (33674)

8 (PDGF or platelet derived growth factor\$).tw. (20842)

9 (EGF or epidermal growth factor\$).tw. (61625)

10 (IGF-I or insulin-like growth factor\$ or IGF1).tw. (45386) 
11 (TGF-a or transforming growth factor alfa or TGFa).tw. (306)

12 (TGF-b or transforming growth factor beta or TGFb).tw. (30559)

13 (EGFR or epidermal growth factor receptor\$).tw. (46446)

14 (VEGF or vascular endothelial growth factor\$).tw. (58203)

15 exp Luteinizing Hormone/bl [Blood] (24870)

16 leptin\$.tw. (26783)

17 exp Progesterone/bl [Blood] (18699)

18 Proteolytic enzyme\$.tw. (9992)

19 exp matrix metalloproteinase 1 / or exp matrix metalloproteinase 2 / or exp matrix metalloproteinase 3 / or exp matrix metalloproteinase 9/ (24504)

20 matrix metalloproteinase\$.tw. (37055)

21 MMP\$.tw. (47849)

22 TIMP\$.tw. (11419)

23 exp "tissue inhibitor of metalloproteinase-1"/ or exp "tissue inhibitor of metalloproteinase-2"/ (6447)

$24 \exp$ Glycoproteins/ (662211)

25 (Ca-125 or Ca125 or cancer antigen 125).tw. (7058)

26 (Ca-19-9 or Ca19-9 or cancer antigen 19-9).tw. (4399)

27 (PP 14 or PP14).tw. (232)

28 serum placental protein\$.tw. (34)

29 exp Follistatin/ (1180)

30 Osteopontin\$.tw. (7267)

31 exp intercellular adhesion molecule-1/ or exp selectins/ (26225)

32 soluble intercellular adhesion.tw. (1663)

33 Soluble adhesion molecule\$.tw. (795)

34 sICAM.tw. (2374)

35 sVCAM\$.tw. (1360)

36 (sEcadherin or soluble E-cadherin).tw. (97)

37 (sEselectin or soluble E-selectin).tw. (713)

38 exp t-lymphocytes/ or exp natural killer t-cells/ (284378)

39 Immune cells alteration\$.tw. (1)

40 (T helper\$ or T supressor\$ or T helper\$ T supressor\$ ratio).tw. (22494)

41 Total complement level\$̣.tw. (24)

42 Autoantibodies.tw. (35161)

43 exp Antibodies, Antiphospholipid/ (7759)

44 Anti-endometrial.tw. (22) 
45 Antiphospholipid\$.tw. (10351)

46 exp hla antigens/ or exp hla-a1 antigen/ or exp hla-a2 antigen/ (66724)

47 (HLA or human leucocyte antigen\$).tw. (83856)

48 Anti-laminin-1.tw. (33)

49 Anti-thyroid.tw. (1478)

50 Anti-Thomsen Friedenreich antigen\$.tw. (8)

51 Anti-transferrin.tw. (284)

52 Anti-LDL.tw. (183)

53 (Anti-2HSG or Heremans-Schmidt glycoprotein).tw. (3)

54 interleukin\$.tw. (184697)

55 (MCP-I or monocyte chemoattractant protein-I).tw. (46)

56 (MIF or migration inhibitory factor\$).tw. (4718)

57 (TNF-a or tumour necrosis factor\$ alfa).tw. (1428)

58 Fas ligand\$.tw. (6204)

59 Endometrial marker\$.tw. (11)

60 CAMs.tw. (1823)

61 cell adhesion molecule\$.tw. (22033)

62 exp Integrins/ (46487)

63 Integrin\$.tw. (42447)

64 Selectin\$.tw. (58540)

65 Cadherin\$.tw. (22688)

66 Aromatase P450.tw. (182)

67 estrogen receptor\$.tw. (41210)

68 progesterone receptor\$.tw. (17437)

69 MTMMP\$.tw. (7)

70 cyr61.tw. (620)

71 exp Cysteine-Rich Protein 61/ (425)

72 cysteine-rich heparin-binding protein\$.tw. (9)

73 (ANXA 1 or ANXA1).tw. (355)

74 (Annexin 1 or Annexin1).tw. (358)

75 (PGP 9?5 or PGP9?5 or protein gene product\$).tw. (2190)

76 serum marker\$.tw. (5721)

77 neural marker\$.tw. (1026)

78 cell surface marker\$.tw. (4751)

79 inflammatory marker\$.tw. (12244) 
80 microarray\$.tw. (81764)

81 microRNA\$.tw. (35967)

82 proteomic\$.tw. (49911)

83 genomic $\$ . t w . ~(205064)$

84 (endometri\$ adj2 biops\$).tw. (3518)

85 Follistatin\$.tw. (1762)

86 Vascular Endothelial Growth Factor A/ (38477)

87 Vitamin D-Binding Protein/ (1356)

88 exp Cytokines/ (575020)

89 exp interleukins/ or exp interleukin-1/ or exp interleukin-6/ or exp interleukin-8/ or exp interleukin-12/ or exp interleukin-13/ (197567)

90 exp Epidermal Growth Factor/ (21875)

91 exp Fibroblast Growth Factors/ (26259)

92 Platelet-Derived Growth Factor/ (11355)

93 Keratin-19/ (1179)

94 exp Clinical Laboratory Techniques/ (2203416)

95 (Luteinizing Hormone\$ or LH).tw. (57796)

96 cytokeratin-19.tw. (1538)

97 (VDBP or vitamin D-binding protein\$).tw. (1262)

98 urinary peptide\$.tw. (148)

99 VDBP-Cr.tw. (1)

100 urinary VDBP corrected for creatinine expression.tw. (1)

101 urinary marker\$.tw. (679)

102 or/1-101 (4283825)

103 Endometriosis/di [Diagnosis] (3449)

104102 or 103 (4286552)

105 exp Endometriosis/ (17833)

106 Endometrio\$.tw. (22478)

107105 or 106 (26003)

108104 and 107 (10936)

109 (animals not (humans and animals)).sh. (4004321)

110108 not 109 (10539)

111 (201501\$ or $201502 \$$ or $201503 \$$ or $201504 \$$ ).ed. (322721)

112110 and 111 (215)

Appendix 2. Search strategy for CENTRAL (OVID platform)

Database: EBM Reviews - Cochrane Central Register of Controlled Trials < July 2015 (3.09.2015)> 
Search Strategy:

1 (biomarker\$ or marker\$).tw. (23692)

2 Laboratory Test\$.tw. (2793)

3 growth factor\$.tw. (5448)

4 scatter factor\$.tw. (8)

5 cytokine\$.tw. (6264)

6 hepatocyte growth factor.tw. (111)

7 (FGF or fibroblast growth factor\$).tw. (433)

8 (PDGF or platelet derived growth factor\$).tw. (250)

9 (EGF or epidermal growth factor\$).tw. (1077)

10 (IGF-I or insulin-like growth factor\$ or IGF1).tw. (2132)

11 (TGF-a or transforming growth factor alfa or TGFa).tw. (519)

12 (TGF-b or transforming growth factor beta or TGFb).tw. (236)

13 (EGFR or epidermal growth factor receptor\$).tw. (1905)

14 (VEGF or vascular endothelial growth factor\$).tw. (1532)

15 exp Luteinizing Hormone/bl [Blood] (151)

16 leptin\$.tw. (1399)

17 exp Progesterone/bl [Blood] (58)

18 Proteolytic enzyme\$.tw. (136)

19 exp matrix metalloproteinase 1 / or exp matrix metalloproteinase 2/ or exp matrix metalloproteinase 3/ or exp matrix metalloproteinase 9/ (292)

20 matrix metalloproteinase\$.tw. (676)

21 MMP\$.tw. (905)

22 TIMP\$.tw. (229)

23 exp "tissue inhibitor of metalloproteinase-1"/ or exp "tissue inhibitor of metalloproteinase-2"/ (101)

24 exp Glycoproteins/ (10108)

25 (Ca-125 or Ca125 or cancer antigen 125).tw. (305)

26 (Ca-19-9 or Ca19-9 or cancer antigen 19-9).tw. (71)

27 (PP 14 or PP14).tw. (23)

28 serum placental protein\$.tw. (6)

29 exp Follistatin/ (13)

30 Osteopontin\$.tw. (80)

31 exp intercellular adhesion molecule-1/ or exp selectins/ (929)

32 soluble intercellular adhesion.tw. (256) 
33 Soluble adhesion molecule\$.tw. (89)

34 sICAM.tw. (319)

35 sVCAM\$.tw. (223)

36 (sEcadherin or soluble E-cadherin).tw. (4)

37 (sEselectin or soluble E-selectin).tw. (99)

38 exp t-lymphocytes/ or exp natural killer t-cells/ (2645)

39 Immune cells alteration\$.tw. (1)

40 (T helper\$ or T supressor\$ or T helper\$ T supressor\$ ratio).tw. (445)

41 Total complement level\$̣.tw. (0)

42 Autoantibodies.tw. (428)

43 exp Antibodies, Antiphospholipid/ (85)

44 Anti-endometrial.tw. (0)

45 Antiphospholipid\$.tw. (152)

46 exp hla antigens/ or exp hla-a1 antigen/ or exp hla-a2 antigen/ (563)

47 (HLA or human leucocyte antigen\$).tw. (1724)

48 Anti-laminin-1.tw. (0)

49 Anti-thyroid.tw. (49)

50 Anti-Thomsen Friedenreich antigen\$.tw. (0)

51 Anti-transferrin.tw. (0)

52 Anti-LDL.tw. (3)

53 (Anti-2HSG or Heremans-Schmidt glycoprotein).tw. (0)

54 interleukin\$..tw. (7276)

55 (MCP-I or monocyte chemoattractant protein-I).tw. (0)

56 (MIF or migration inhibitory factor\$).tw. (75)

57 (TNF-a or tumour necrosis factor\$ alfa).tw. (3923)

58 Fas ligand\$.tw. (47)

59 Endometrial marker\$.tw. (2)

60 CAMs.tw. (53)

61 cell adhesion molecule\$.tw. (568)

62 exp Integrins/ (781)

63 Integrin\$.tw. (248)

64 Selectin\$.tw. (2183)

65 Cadherin\$.tw. (71)

66 Aromatase P450.tw. (3)

67 estrogen receptor\$.tw. (1252) 
68 progesterone receptor\$.tw. (531)

69 MTMMP\$.tw. (0)

70 cyr61.tw. (1)

71 exp Cysteine-Rich Protein 61/ (1)

72 cysteine-rich heparin-binding protein\$.tw. (0)

73 (ANXA 1 or ANXA1).tw. (3)

74 (Annexin 1 or Annexin1).tw. (2)

75 (PGP 9?5 or PGP9?5 or protein gene product\$).tw. (18)

76 serum marker\$.tw. (411)

77 neural marker\$.tw. (9)

78 cell surface marker\$.tw. (46)

79 inflammatory marker\$.tw. (1739)

80 microarray\$.tw. (501)

81 microRNA\$.tw. (103)

82 proteomic\$.tw. (176)

83 genomic\$.tw. (526)

84 (endometri\$ adj2 biops\$).tw. (464)

85 Follistatin\$.tw. (26)

86 Vascular Endothelial Growth Factor A/ (560)

87 Vitamin D-Binding Protein/ (18)

88 exp Cytokines/ (13960)

89 exp interleukins/ or exp interleukin-1/ or exp interleukin-6/ or exp interleukin-8/ or exp interleukin-12/ or exp interleukin-13/ (4413)

90 exp Epidermal Growth Factor/ (91)

91 exp Fibroblast Growth Factors/ (197)

92 Platelet-Derived Growth Factor/ (99)

93 Keratin-19/ (19)

$94 \exp$ Clinical Laboratory Techniques/ (35164)

95 (Luteinizing Hormone\$ or LH).tw. (2935)

96 cytokeratin-19.tw. (25)

97 (VDBP or vitamin D-binding protein\$).tw. (44)

98 urinary peptide\$.tw. (8)

99 VDBP-Cr.tw. (0)

100 urinary VDBP corrected for creatinine expression.tw. (0)

101 urinary marker\$.tw. (67)

102 or/1-101 (90390) 
103 Endometriosis/di [Diagnosis] (6)

104102 or 103 (90394)

$105 \exp$ Endometriosis/ (469)

106 Endometrio\$.tw. (1026)

107105 or $106(1067)$

108104 and 107 (226)

109 (animals not (humans and animals)).sh. (1)

110108 not 109 (226)

\section{Appendix 3. Search strategy for EMBASE (OVID platform)}

Database: EMBASE (Ovid) <1980 to 2015 Week 07 (16.02.2015)>

Search strategy:

1 Laboratory Test\$̦.tw. (41662)

2 growth factor\$.tw. (318593)

3 scatter factor\$.tw. (1388)

4 cytokine\$.tw. (322134)

5 hepatocyte growth factor.tw. (9594)

6 (FGF or fibroblast growth factor\$).tw. (37191)

7 (PDGF or platelet derived growth factor\$).tw. (23530)

8 (EGF or epidermal growth factor\$).tw. (69553)

9 (IGF-I or insulin-like growth factor\$ or IGF1).tw. (49806)

10 (TGF-a or transforming growth factor alfa or TGFa).tw. (542)

11 (TGF-b or transforming growth factor beta or TGFb).tw. (30820)

12 (EGFR or epidermal growth factor receptor\$).tw. (64664)

13 (VEGF or vascular endothelial growth factor\$).tw. (73191)

14 exp luteinizing hormone/ec [Endogenous Compound] (21924)

15 leptin\$.tw. (32576)

16 exp progesterone blood level/ or exp progesterone urine level/ (6285)

17 Proteolytic enzyme\$.tw. (9643)

18 exp matrix metalloproteinase/ (19364)

19 matrix metalloproteinase\$.tw. (41445)

20 MMP\$.tw. (58466)

21 TIMP\$.tw. (14174)

22 exp "tissue inhibitor of metalloproteinase 2"/ (4824)

23 exp "tissue inhibitor of metalloproteinase 1"/ (8779) 
24 exp glycoprotein/ec [Endogenous Compound] (246077)

25 (Ca-125 or Ca125 or cancer antigen 125).tw. (9536)

26 (Ca-19-9 or Ca19-9 or cancer antigen 19-9).tw. (6054)

27 (PP 14 or PP14).tw. (244)

28 serum placental protein\$.tw. (43)

29 exp follistatin/ (2148)

30 Osteopontin\$.tw. (8475)

31 exp intercellular adhesion molecule 1/ (32066)

32 exp selectin/ (3082)

33 soluble intercellular adhesion.tw. (1788)

34 Soluble adhesion molecule\$.tw. (919)

35 sICAM.tw. (2888)

36 sVCAM\$.tw. (1793)

37 (sEcadherin or soluble E-cadherin).tw. (120)

38 (sEselectin or soluble E-selectin).tw. (822)

39 exp T lymphocyte/ (374675)

40 exp natural killer T cell/ (5800)

41 Immune cells alteration\$.tw. (6)

42 (T helper\$ or T supressor $\$$ or T helper\$ T supressor\$ ratio).tw. (24786)

43 Total complement level\$.tw. (20)

44 Autoantibodies.tw. (42037)

45 exp phospholipid antibody/ (9920)

46 Anti-endometrial.tw. (23)

47 Antiphospholipid\$.tw. (13777)

48 exp HLA antigen/ (81011)

49 exp HLA A1 antigen/ (597)

50 exp HLA A2 antigen/ (3288)

51 (HLA or human leucocyte antigen\$).tw. (104497)

52 Anti-laminin-1.tw. (43)

53 Anti-thyroid.tw. (1873)

54 Anti-Thomsen Friedenreich antigen\$.tw. (5)

55 Anti-transferrin.tw. (290)

56 Anti-LDL.tw. (186)

57 (Anti-2HSG or Heremans-Schmidt glycoprotein).tw. (4)

58 interleukin\$.tw. (199692) 
59 (MCP-I or monocyte chemoattractant protein-I).tw. (112)

60 (MIF or migration inhibitory factor\$).tw. (5063)

61 (TNF-a or tumour necrosis factor\$ alfa).tw. (5998)

62 Fas ligand\$.tw. (6708)

63 Endometrial marker\$.tw. (18)

64 CAMs.tw. (2100)

65 cell adhesion molecule\$.tw. (24039)

66 exp integrin/ (29036)

67 Integrin\$.tw. (48293)

68 Selectin\$.tw. (67300)

69 Cadherin\$.tw. (27150)

70 Aromatase P450.tw. (202)

71 estrogen receptor\$.tw. (46656)

72 progesterone receptor\$.tw. (19861)

73 MTMMP\$.tw. (15)

74 cyr61.tw. (755)

75 exp cysteine rich protein 61/ (753)

76 cysteine-rich heparin-binding protein\$.tw. (12)

77 (ANXA 1 or ANXA1).tw. (452)

78 (Annexin 1 or Annexin1).tw. (425)

79 (PGP 9?5 or PGP9?5 or protein gene product\$).tw. (2620)

80 serum marker\$.tw. (7720)

81 neural marker\$.tw. (1119)

82 cell surface marker\$.tw. (5851)

83 inflammatory marker\$.tw. (17339)

84 microarray\$.tw. (101846)

85 microRNA\$.tw. (40082)

86 proteomic\$.tw. (55191)

87 genomic\$.tw. (217184)

88 (endometri\$ adj2 biops\$).tw. (4369)

89 Follistatin\$.tw. (1945)

90 exp vasculotropin/ (69810)

91 Vascular Endothelial Growth Factor A.tw. (2275)

92 exp vitamin D binding protein/ (2064)

93 exp cytokine/ (1034772) 
94 exp interleukin derivative/ (2790)

95 exp interleukin 1/ (48499)

96 exp interleukin 6/ (136328)

97 exp interleukin 8/ (48884)

98 exp interleukin 12/ (31842)

99 exp interleukin 13/ (13584)

100 exp epidermal growth factor/ (32130)

101 exp fibroblast growth factor/ (13858)

102 cytokeratin 19/ (3601)

103 platelet derived growth factor/ (18930)

104 cytokeratin-19.tw. (1918)

105 (VDBP or vitamin D-binding protein\$).tw. (1413)

106 urinary peptide\$.tw. (174)

107 VDBP-Cr.tw. (1)

108 urinary VDBP corrected for creatinine expression.tw. (1)

109 urinary marker\$.tw. (830)

110 exp blood analysis/ (118854)

111 exp endometrium biopsy/ (4988)

112 exp urinalysis/ or exp biological marker/ (210153)

113 (biomarker or biomarkers).tw. (159748)

114 or/1-113 (2734501)

115 endometriosis/di [Diagnosis] (4979)

116114 or 115 (2738583)

117 exp endometriosis/ (25923)

118 Endometriosis.tw. (22110)

119117 or 118 (27911)

120116 and 119 (10326)

121 Animal/ not Human/ (1204497)

122120 not 121 (10279)

\section{Additional search February 2015 - May 2015}

Embase <1980 to 2015 Week 35 (3.09.2015)>

Search Strategy:

1 Laboratory Test\$.tw. (44290)

2 growth factor\$.tw. (335543) 
3 scatter factor\$.tw. (1407)

4 cytokine\$.tw. (343623)

5 hepatocyte growth factor.tw. (10104)

6 (FGF or fibroblast growth factor\$).tw. (39159)

7 (PDGF or platelet derived growth factor\$).tw. (24591)

8 (EGF or epidermal growth factor\$).tw. (73599)

9 (IGF-I or insulin-like growth factor\$ or IGF1).tw. (51838)

10 (TGF-a or transforming growth factor alfa or TGFa).tw. (583)

11 (TGF-b or transforming growth factor beta or TGFb).tw. (32580)

12 (EGFR or epidermal growth factor receptor\$).tw. (71526)

13 (VEGF or vascular endothelial growth factor\$).tw. (79087)

14 exp luteinizing hormone/ec [Endogenous Compound] (22767)

15 leptin\$.tw. (34921)

16 exp progesterone blood level/ or exp progesterone urine level/ (6534)

17 Proteolytic enzyme\$.tw. (9903)

18 exp matrix metalloproteinase/ (20462)

19 matrix metalloproteinase\$.tw. (44380)

20 MMP\$.tw. (63208)

21 TIMP\$.tw. (15146)

22 exp "tissue inhibitor of metalloproteinase 2"/ (5136)

23 exp "tissue inhibitor of metalloproteinase 1"/ (9381)

24 exp glycoprotein/ec [Endogenous Compound] (260024)

25 (Ca-125 or Ca125 or cancer antigen 125).tw. (10051)

26 (Ca-19-9 or Ca19-9 or cancer antigen 19-9).tw. (6446)

27 (PP 14 or PP14).tw. (243)

28 serum placental protein\$.tw. (44)

29 exp follistatin/ (2283)

30 Osteopontin\$.tw. (9173)

31 exp intercellular adhesion molecule 1/ (33492)

32 exp selectin/ (3217)

33 soluble intercellular adhesion.tw. (1865)

34 Soluble adhesion molecule\$.tw. (944)

35 sICAM.tw. (3049)

36 sVCAM\$.tw. (1924)

37 (sEcadherin or soluble E-cadherin).tw. (125) 
38 (sEselectin or soluble E-selectin).tw. (861)

39 exp T lymphocyte/ (394405)

40 exp natural killer T cell/ (6310)

41 Immune cells alteration\$.tw. (6)

42 (T helper\$ or T supressor\$ or T helper\$ T supressor\$ ratio).tw. (26082)

43 Total complement level\$.tw. (20)

44 Autoantibodies.tw. (44153)

45 exp phospholipid antibody/ (10362)

46 Anti-endometrial.tw. (25)

47 Antiphospholipid\$.tw. (14399)

48 exp HLA antigen/ (83748)

49 exp HLA A1 antigen/ (622)

50 exp HLA A2 antigen/ (3409)

51 (HLA or human leucocyte antigen\$).tw. (109332)

52 Anti-laminin-1.tw. (43)

53 Anti-thyroid.tw. (2059)

54 Anti-Thomsen Friedenreich antigen\$.tw. (7)

55 Anti-transferrin.tw. (297)

56 Anti-LDL.tw. (191)

57 (Anti-2HSG or Heremans-Schmidt glycoprotein).tw. (4)

58 interleukin\$.tw. (210083)

59 (MCP-I or monocyte chemoattractant protein-I).tw. (114)

60 (MIF or migration inhibitory factor\$).tw. (5342)

61 (TNF-a or tumour necrosis factor\$ alfa).tw. (6488)

62 Fas ligand\$.tw. (6895)

63 Endometrial marker\$.tw. (18)

64 CAMs.tw. (2198)

65 cell adhesion molecule\$.tw. (25207)

66 exp integrin/ (30330)

67 Integrin\$s.tw. (50938)

68 Selectin\$.tw. (71624)

69 Cadherin\$.tw. (29496)

70 Aromatase P450.tw. (207)

71 estrogen receptor\$.tw. (49530)

72 progesterone receptor\$.tw. (21068) 
73 MTMMP\$.tw. (16)

74 cyr61.tw. (822)

75 exp cysteine rich protein 61/ (829)

76 cysteine-rich heparin-binding protein\$.tw. (12)

77 (ANXA 1 or ANXA1).tw. (500)

78 (Annexin 1 or Annexin1).tw. (440)

79 (PGP 9?5 or PGP9?5 or protein gene product\$).tw. (2760)

80 serum marker\$.tw. (8158)

81 neural marker\$.tw. (1234)

82 cell surface marker\$.tw. (6222)

83 inflammatory marker\$.tw. (19492)

84 microarray\$.tw. (110181)

85 microRNA\$..tw. (47554)

86 proteomic\$.tw. (60599)

87 genomic\$.tw. (233444)

88 (endometri\$ adj2 biops\$).tw. (4589)

89 Follistatin\$.tw. (2081)

90 exp vasculotropin/ (74115)

91 Vascular Endothelial Growth Factor A.tw. (2526)

92 exp vitamin D binding protein/ (2196)

93 exp cytokine/ (1094317)

94 exp interleukin derivative/ (3281)

95 exp interleukin 1/ (50850)

96 exp interleukin 6/ (147379)

97 exp interleukin 8/ (52281)

98 exp interleukin 12/ (33479)

99 exp interleukin 13/ (14685)

100 exp epidermal growth factor/ (33057)

101 exp fibroblast growth factor/ (14499)

102 cytokeratin 19/ (3886)

103 platelet derived growth factor/ (19655)

104 cytokeratin-19.tw. (2030)

105 (VDBP or vitamin D-binding protein\$).tw. (1520)

106 urinary peptide\$.tw. (189)

107 VDBP-Cr.tw. (1) 
108 urinary VDBP corrected for creatinine expression.tw. (1)

109 urinary marker\$.tw. (883)

110 exp blood analysis/ (124468)

111 exp endometrium biopsy/ (5197)

112 exp urinalysis/ or exp biological marker/ (232619)

113 (biomarker or biomarkers).tw. (182609)

114 or/1-113 (2911073)

115 endometriosis/di [Diagnosis] (5173)

116114 or 115 (2915302)

117 exp endometriosis/ (27433)

118 Endometriosis.tw. (23449)

119117 or 118 (29532)

120116 and 119 (10922)

121 Animal/ not Human/ (1261620)

122120 not 121 (10862)

123 (201501\$ or $201502 \$$ or $201503 \$$ or $201504 \$$ ).em. (49200)

124122 and 123 (34)

Appendix 4. Search strategy for CINAHL database (EBSCO platform)

Database: CINAHL Plus with Full Text (EBSCOhost) <1980 to 20.04.2015>

Search strategy:

\begin{tabular}{lll}
\hline$\#$ & Query & Results \\
\hline S97 & S3 AND S96 & 1131 \\
\hline S96 & S4 OR S5 OR S6 OR S7 OR S8 OR S9 OR S10 OR S11 OR S12 OR S13 OR S14 OR & 341775 \\
& S15 OR S16 OR S17 OR S18 OR S19 OR S20 OR S21 OR S22 OR S23 OR S24 OR & \\
& S25 OR S26 OR S27 OR S28 OR S29 OR S30 OR S31 OR S32 OR S33 OR S34 OR \\
& S35 OR S36 OR S37 OR S38 OR S39 OR S40 OR S41 OR S42 OR S43 OR S44 OR & \\
& S45 OR S46 OR S47 OR S48 OR S49 OR S50 OR S51 OR S52 OR S53 OR S54 OR & \\
& S55 OR S56 OR S57 OR S58 OR S59 OR S60 OR S61 OR S62 OR S63 OR S64 OR & \\
& S65 OR S66 OR S67 OR S68 OR S69 OR S70 OR S71 OR S72 OR S73 OR S74 OR & 1598 \\
\hline S75 OR S76 OR S77 OR S78 OR S79 OR S80 OR S81 OR S82 OR S83 OR S84 OR & 134 \\
\hline S95 & S95 OR S86 OR S87 OR S88 OR S89 OR S90 OR S91 OR S92 OR S93 OR S94 OR & \\
\hline S94 & TX urinary peptide* & 109 \\
\hline S93 & TX (VDBP or vitamin D-binding protein*) & 18041 \\
\hline S92 & TX cytokeratin-19 & \\
\hline
\end{tabular}


(Continued)

\begin{tabular}{|c|c|c|}
\hline S91 & (MH "Diagnosis, Laboratory+") & 101773 \\
\hline S90 & "Keratin-19" & 2 \\
\hline S89 & (MH "Platelet-Derived Growth Factor") & 394 \\
\hline S88 & (MH "Epidermal Growth Factors") & 1264 \\
\hline S87 & (MH "Interleukins") & 6584 \\
\hline S86 & (MH "Cytokines") & 6860 \\
\hline S85 & TX Vitamin D-Binding Protein & 131 \\
\hline S84 & (MH "Vascular Endothelial Growth Factor A") & 194 \\
\hline S83 & TX (endometri* N2 biops*) & 432 \\
\hline S82 & TX (endometri* adj2 biops) & 0 \\
\hline S81 & TX genomic\$ & 7487 \\
\hline S80 & TX proteomic* & 2434 \\
\hline S79 & TX microRNA & 824 \\
\hline S78 & TX microarray & 3123 \\
\hline S77 & TX (PGP 95 or PGP95 or protein gene product ${ }^{\star}$ ) & 9925 \\
\hline S76 & TX (Annexin 1 or Annexin 1 ) & 472 \\
\hline S75 & TX (ANXA 1 or ANXA1) & 41 \\
\hline S74 & TX cysteine-rich heparin-binding protein* & 12 \\
\hline S73 & (MH "Protein Array Analysis") & 73 \\
\hline S72 & TX cyr61 & 34 \\
\hline S71 & TX MTMMP* & 0 \\
\hline S70 & TX progesterone receptor ${ }^{\star}$ & 1927 \\
\hline S69 & TX estrogen receptor* & 5193 \\
\hline S68 & TX Aromatase P450 & 38 \\
\hline S67 & TX Cadherin* & 900 \\
\hline S66 & TX Selectin* & 28411 \\
\hline S65 & TX Integrin* & 1587 \\
\hline S64 & TX cell adhesion molecule* & 1578 \\
\hline
\end{tabular}


(Continued)

\begin{tabular}{|c|c|c|}
\hline S63 & TX CAMs & 550 \\
\hline S62 & TX Endometrial marker* & 54 \\
\hline S61 & TX Fas ligand & 338 \\
\hline S60 & TX (TNF-a or tumour necrosis factor ${ }^{\star}$ alfa) & 1489 \\
\hline S59 & TX (MIF or migration inhibitory factor ${ }^{\star}$ ) & 399 \\
\hline S58 & TX (MCP-I or monocyte chemoattractant protein-I) & 13 \\
\hline S57 & TX interleukin & 13809 \\
\hline S56 & TX (Anti-2HSG or Heremans-Schmidt glycoprotein) & 7 \\
\hline S55 & TX Anti-LDL & 9 \\
\hline S54 & TX Anti-transferrin & 3 \\
\hline S53 & TX Anti-Thomsen Friedenreich antigen* & 1 \\
\hline S52 & TX Anti-thyroid & 109 \\
\hline S51 & TX Anti-laminin-1 & 15 \\
\hline S50 & TX (HLA or human leucocyte antigen $\left.{ }^{\star}\right)$ & 4202 \\
\hline S49 & (MM "HLA Antigens") & 638 \\
\hline S48 & TX Antiphospholipid* & 1249 \\
\hline S47 & TX Anti-endometrial & 34 \\
\hline S46 & (MH "Antibodies/BL/DU") & 1294 \\
\hline S45 & TX Autoantibodies & 4385 \\
\hline S43 & TX Total complement level & 3 \\
\hline S42 & TX (T helper ${ }^{\star}$ or $T$ supressor ${ }^{\star}$ ) & 2341 \\
\hline S41 & TX Immune cells alteration* & 24 \\
\hline S40 & TX natural killer t-cells & 669 \\
\hline S39 & (MM "T Lymphocytes") & 2404 \\
\hline S38 & TX (sEselectin or soluble E-selectin) & 91 \\
\hline S37 & TX (sEcadherin or soluble E-cadherin) & 8 \\
\hline S36 & TX sVCAM & 100 \\
\hline S35 & TX SICAM & 173 \\
\hline
\end{tabular}




\begin{tabular}{|c|c|c|}
\hline S34 & TX Soluble adhesion molecule & 368 \\
\hline S33 & TX soluble intercellular adhesion & 237 \\
\hline S32 & (MM "Cell Adhesion Molecules") & 52 \\
\hline S31 & TX Osteopontin ${ }^{\star}$ & 416 \\
\hline S30 & TX Follistatin & 74 \\
\hline S29 & TX serum placental protein* & 11 \\
\hline S28 & TX (Ca-19-9 or Ca19-9 or cancer antigen 19-9) & 262 \\
\hline S27 & TX (Ca-125 or Ca125 or cancer antigen 125) & 831 \\
\hline S26 & (MM "Glycoproteins/BL/DU") & 224 \\
\hline S25 & TX tissue inhibitor of metalloproteinase & 423 \\
\hline S24 & TX TIMP* & 1845 \\
\hline S23 & $\mathrm{TXMMP}^{\star}$ & 4244 \\
\hline S22 & TX matrix metalloproteinase ${ }^{\star}$ & 3325 \\
\hline S21 & TX Proteolytic enzyme* & 1461 \\
\hline S20 & (MM "Progesterone/BL/DU") & 51 \\
\hline S19 & TX leptin* & 3258 \\
\hline S18 & (MM "Luteinizing Hormone/BL/DU") & 38 \\
\hline S17 & TX (VEGF or vascular endothelial growth factor ${ }^{\star}$ ) & 7166 \\
\hline S16 & TX (EGFR or epidermal growth factor receptor ${ }^{\star}$ ) & 6188 \\
\hline S15 & TX (TGF-b or transforming growth factor beta or TGFb) & 2972 \\
\hline S14 & TX (TGF-a or transforming growth factor alfa or TGFa) & 464 \\
\hline S13 & TX (IGF-I or insulin-like growth factor ${ }^{\star}$ or IGF1) & 3588 \\
\hline S12 & TX (EGF or epidermal growth factor ${ }^{\star}$ ) & 6250 \\
\hline S11 & TX (PDGF or platelet derived growth factor ${ }^{\star}$ ) & 3195 \\
\hline S10 & TX (FGF or fibroblast growth factor ${ }^{\star}$ ) & 3395 \\
\hline S9 & TX hepatocyte growth factor ${ }^{\star}$ & 880 \\
\hline S8 & TX cytokine* & 20821 \\
\hline S7 & TX scatter factor ${ }^{\star}$ & 1864 \\
\hline
\end{tabular}


(Continued)

\begin{tabular}{lll} 
S6 & TX growth factor & 76163 \\
\hline S5 & TX Laboratory Test* & 82732 \\
\hline S4 & TX (biomarker* ${\left.\text { or marker }{ }^{\star}\right)}^{*}$ & 84857 \\
\hline S3 & S1 OR S2 & 2841 \\
\hline S2 & TX Endometrio* & 2841 \\
\hline S1 & (MM "Endometriosis") & 889 \\
\hline S4 & TX (biomarker* or marker $)$ & 61,794 \\
\hline S3 & S1 OR S2 & 2,174 \\
\hline S2 & TX Endometrio* & 2,174 \\
\hline S1 & (MM "Endometriosis") & 1,306 \\
\hline
\end{tabular}

\section{Appendix 5. Search strategy for other databases}

\section{Search for clinical studies}

Database: Web of Science Core Collection (Thomson Reuters) $<1900$ to Present $(\mathbf{2 0 . 0 4 . 2 0 1 5 ) >}$

Search strategy:

1. Topic $=\left(\right.$ endometrio $\left.{ }^{\star}\right)$ AND Topic $=\left(\right.$ diagnos $^{\star}$ OR test ${ }^{\star}$ OR marker ${ }^{\star}$ OR biomarker $\left.{ }^{\star}\right) ;$ Timespan=All Years $(7425)$

Database: PsycINFO (Ovid) <1806 to April Week 22015 (20.04.2015)>

Search strategy:

1. endometriosis.tw. (174)

\section{Database: LILACS <20.04.2015>}

Search strategy:

1. (tw:(endometriosis)) AND (tw:(diagnos $\left.\left.{ }^{\star}\right)\right)(420)$

Database: OAlster (WorldCat.org) <20.04.2015>

Search strategy:

1. endometriosis and (marker ${ }^{\star}$ or biomarker $\left.{ }^{\star}\right)(11)$

2. endometriosis and diagnos* $(446)$

\section{Database: TRIP <20.04.2015>}

Search strategy:

1. (endometriosis and diagnos $\left.{ }^{\star}\right)(1648)$

Searches of trial registers for ongoing and registered trials

Database: 'ClinicalTrials.gov', a service of the US national Institute of Health

Search strategy: 
1. endometriosis (220)

2. endometriosis AND diagnosis (22)

Database: WHO International Clinical Trials Registry Platform (ICTRP) <20.04.2015>

Search strategy:

1. endometriosis (523)

Searches for the reviews as potential source of references

Database: MEDION <10.01.2014>

Search strategy:

ICP Code female genital system (including breast), Signssymp medical imaging, laboratory tests, histology and cytology, endoscopy and laparoscopy. Filter: systematic reviews of diagnostic studies (2)

Database: DARE (CRD) $<20.04 .2015>$

Search strategy:

1. endometriosis (99)

PubMed, a 'Systematic Review' search under the 'Clinical Queries' link <20.04.2015>

Search strategy:

(endometriosis) AND systematic[sb] (418)

Category: Diagnosis; Scope: Broad

Searches for the papers recently published and not yet indexed in the major databases

Search engine: PubMed <20.10.2014 to 20.04.2015>

Search strategy:
1. marker (14979)
2. test (61151)
3. diagnos $^{\star}(69743)$
4. biomarker (10806)
5. or/1-4 (7943)

Index test(s) set

Filters: Publication date from $2014 / 10 / 20$ to $2015 / 04 / 20$

6. Endometriosis (584)

Target condition set

Filters: Publication date from $2014 / 10 / 20$ to $2015 / 04 / 20$

\section{5 and $6(267)$}

Combined sets

Filters: Publication date from $2014 / 10 / 20$ to $2015 / 04 / 20$

\section{CONTRIBUTIONS OF AUTHORS}

Vicki Nisenblat and Louise Hull coordinated the production of the protocol and produced the first draft. Emily Liu and Vicki Nisenblat undertook the review and took the primary role in writing with equal contribution to the review. Louise Hull was actively involved in quality 
appraisal and data extraction for the included studies and took the primarily role in editing the review. Patrick Bossuyt provided advice on statistical methods for the review. Cindy Farquhar critically reviewed the methodological aspects and participated in the study design. Ian Fraser and Neil Johnson contributed to the conception and the design of the review. All the authors contributed to the revision and drafting of the protocol and the review.

\section{DECLARATIONS OF INTEREST}

All authors declared no conflicts of interest.

\section{SOURCES OF SUPPORT}

\section{Internal sources}

- Cochrane Gynaecology and Fertility Group, the University of Auckland, New Zealand.

Technical support

- The Robinson Institute, the University of Adelaide, Australia.

Access to academic resources

\section{External sources}

- No sources of support supplied

\section{DIFFERENCES BETWEEN PROTOCOLANDREVIEW}

General scope: this review is a part of the review series arising from the same generic protocol. The following sections were adjusted to the main topic of the review as following:

Background: the section on the index test was modified and only information pertaining to urine tests was retained. The 'Rationale' section was updated and now includes a more detailed definition of triage diagnostic tests.

Objectives:

- Substantial numbers of studies revealed biomarkers with expression levels which were not altered by the presence of endometriosis (no statistically significant difference was found between women with and without the disease). These data were included when obtained from the adequately designed studies, the rationale for this being explained in the Background section under 'Rationale', in the Methods section under 'Criteria for considering studies for this review - Index test' and added to 'Objectives' as a secondary objective: '2. To assess the biomarkers which were not affected by endometriosis and hence were unlikely to discriminate between women with and without the disease'.

- The list of the sources of heterogeneity has been updated.

Methods:

- Criteria for considering studies for this review were updated as following:

- $\quad$ Types of studies: we removed the 'cohort' and 'case control' classifications and introduced the concepts of 'single-gate design' and 'two-gate design'. This was defined as the presence of a single or multiple set of inclusion criteria by clinical condition or by reference standard. We found this classification more informative in the description of diagnostic studies, all of which are cross-sectional in nature. We limited the inclusion criteria to the studies with a single set of inclusion criteria by reference standard (i.e. all women who underwent abdominal surgery), but included single or multiple sets of inclusion criteria by clinical presentation (i.e. women with suspected endometriosis or other indications for abdominal surgery), referring to these as 'single-gate design' and 'two-gate design', respectively.

- Likewise, we removed the terminology 'prospective studies' and introduced 'studies performed on prospectively collected samples'. This decision was guided by the fact that most diagnostic studies are retrospective in nature, as they aim to compare the result of index test with the result of reference standard in the same group of participants, where the groups are classified by the outcome of reference standard. Also, the analysis of the index test could have been performed retrospectively in a single batch on stored samples after the prospective collection of samples. The timing of sample collection (before or after surgical treatment of the disease) from a pre-operatively recruited population has more impact on the test result than the timing of the laboratory assay. Therefore, we included only studies where urine was collected before the reference surgical procedure, i.e. 'prospectively collected', irrespective of actual timing of test performance and abandoned labelling studies as 'prospective' or 'retrospective' to avoid confusion. This allowed us to include the studies from well-established high quality tissue banks using well-characterised archived samples, as omission of these studies would have resulted in the loss of potentially valuable data. This is presented in 'Methods' under 'Criteria for considering studies for this review'. 
- Index tests were modified to pertain only to urinary biomarkers and the table listing the tests of interest was updated accordingly (Table 2).

- Target conditions now also includes deep pelvic endometriosis in view of the growing body of literature on this condition as a separate entity and its diagnostic importance to optimise the surgical approach.

- Spectrum of disease: following an ad hoc observation, we included the studies that involved only selected populations of women with endometriosis (i.e. specific rASRM stages) in view of the emerging evidence on poor correlation of this classification with infertility and pain symptoms. Exclusion of such studies could result in the loss of potentially important diagnostic information from the otherwise eligible publications. Where possible we aimed to address the impact of the inclusion of these studies in investigations of heterogeneity.

- Search methods for identification of studies:

- In the protocol we stated that the grey literature (unpublished studies including conference proceedings and reports) would be identified, and defined specific search strategies. In practice, the paucity of relevant data that was available from abstracts made it impossible to apply the selection criteria and methodological quality judgements. Therefore, by consensus between the key authors, we removed already identified unpublished studies and did not complete an intended search for unpublished material.

- The search strings were updated for all biomarkers excluding imaging (searched separately), applying the same principles as presented in the protocol.

- Assessment of methodological quality: the QUADAS-2 tool was tailored for the topic of the review. The differences between the original QUADAS-2 tool and the one designed for this review are outlined in the relevant section in 'Methods'.

Analysis:

- The section on statistical methods was amended and tailored to the types of tests included in the review.

- We performed no sensitivity analyses and no assessment of heterogeneity due to insufficient data.

- When test performance was judged against the predetermined diagnostic criteria, the point estimates of sensitivity and specificity were considered as the most informative presentation of test performance. We acknowledge that tests with point estimates that did not reach the predetermined criteria but confidence intervals (CIs) which contained values above the threshold, could have diagnostic value. Furthermore tests with point estimates that reached the criteria but Cls which contained values below the threshold, could have an overestimated diagnostic value. If the range of the Cls rather than the point estimates of the data are used, the predetermined cut-off becomes meaningless. Therefore we did not consider Cls in qualifying the test performance, but utilised this information in interpreting the reliability of the obtained data.

\section{NOTES}

The initially planned single review on the non-invasive tests for diagnosis of endometriosis was split into several smaller reviews in order to facilitate data handling and interpretation, due to the abundance and diversity of the suggested tests. The review was generated from a generic protocol, which was designed for all the reviews in this series. The other reviews from the series include 1) Endometrial biomarkers for the non-invasive diagnosis of endometriosis; 2) Urinary biomarkers for the non-invasive diagnosis of endometriosis; 3) Imaging modalities for the non-invasive diagnosis of endometriosis; 4) Combined biomarkers for the non-invasive diagnosis of endometriosis.

\section{NDEX TERMS}

\section{Medical Subject Headings (MeSH)}

Biomarkers [urine]; Diagnosis, Differential; Endometriosis [*diagnosis]; Keratin-19 [*urine]; Peptide Fragments [urine]; Peptides [*urine]; Phosphopyruvate Hydratase [ ${ }^{\star}$ urine]; Proteomics; Vitamin D-Binding Protein [ ${ }^{\star}$ urine]

\section{MeSH check words}

Female; Humans 